\title{
A FULLY-MIXED FORMULATION IN BANACH SPACES FOR THE COUPLING OF THE STEADY BRINKMAN-FORCHHEIMER AND DOUBLE-DIFFUSION EQUATIONS
}

\author{
Sergio Caucao ${ }^{1, *} \odot$, Gabriel N. Gatica ${ }^{2}$ and Juan P. Ortega ${ }^{2}$
}

\begin{abstract}
We propose and analyze a new mixed finite element method for the nonlinear problem given by the coupling of the steady Brinkman-Forchheimer and double-diffusion equations. Besides the velocity, temperature, and concentration, our approach introduces the velocity gradient, the pseudostress tensor, and a pair of vectors involving the temperature/concentration, its gradient and the velocity, as further unknowns. As a consequence, we obtain a fully mixed variational formulation presenting a Banach spaces framework in each set of equations. In this way, and differently from the techniques previously developed for this and related coupled problems, no augmentation procedure needs to be incorporated now into the formulation nor into the solvability analysis. The resulting non-augmented scheme is then written equivalently as a fixed-point equation, so that the well-known Banach theorem, combined with classical results on nonlinear monotone operators and Babuška-Brezzi's theory in Banach spaces, are applied to prove the unique solvability of the continuous and discrete systems. Appropriate finite element subspaces satisfying the required discrete inf-sup conditions are specified, and optimal a priori error estimates are derived. Several numerical examples confirm the theoretical rates of convergence and illustrate the performance and flexibility of the method.
\end{abstract}

Mathematics Subject Classification. 5N30, 65N12, 65N15, 35Q79, 76R05, 76 D07.

Received May 27, 2021. Accepted October 27, 2021.

\section{INTRODUCTION}

The phenomenon in which two scalar fields, such as heat and concentration of a solute, affect the density distribution in a fluid-saturated porous medium, referred to as double-diffusive convection, is a challenging multiphysics problem. This model has numerous applications, among which we highlight predicting and controlling processes arising in geophysics, oceanography, chemical engineering, and energy technology, to name a few areas of interest. In particular, some of them include groundwater system in karst aquifers, chemical processing, simulation of bacterial bioconvection and thermohaline circulation problems, convective flow of carbon nanotubes, and propagation of biological fluids (see, e.g. $[1,6,9,22,32]$ ). In this regard, we remark that much of the research

\footnotetext{
Keywords and phrases. Brinkman-Forchheimer equations, double-diffusion equations, fully-mixed formulation, fixed point theory, mixed finite element methods, a priori error analysis.

1 Departamento de Matemática y Física Aplicadas, Universidad Católica de la Santísima Concepción, Casilla 297, Concepción, Chile.

$2 \mathrm{CI}^{2} \mathrm{MA}$ and Departamento de Ingeniería Matemática, Universidad de Concepción, Casilla 160-C, Concepción, Chile.

${ }^{*}$ Corresponding author: scaucao@ucsc.cl.
} 
in porous medium has been focused on the use of Darcy's law. However, this constitutive equation becomes unreliable to model the flow of fluids through highly porous media with Reynolds numbers greater than one, as in the above applications. To overcome this limitation, a first alternative is to employ the Brinkman model [8], which describes Stokes flows through a set of obstacles, and therefore can be applied precisely to that kind of media. Another possible option is the Forchheimer law [23], which accounts for faster flows by including a nonlinear inertial term. According to the above, the Brinkman-Forchheimer equation (see, e.g. [12,16]), which combines the advantages of both models, has been used for fast flows in highly porous media. Moreover, this fact has motivated the introduction of the corresponding coupling with the so called double-diffusion equations (a system of advection-diffusion equations), through convective terms and the body force.

To the authors' knowledge, one of the first works analyzing the coupling of the incompressible BrinkmanForchheimer and double-diffusion equations is [28], where well-posedness and regularity of solution for a velocitypressure-temperature-concentration variational formulation is established by combining the Galerkin method with a smallness data assumption. Later on, the global solvability of the coupling of the unsteady double-diffusive convection system under homogeneous Neumann boundary conditions and a linearized version of the BrinkmanForchheimer equations, was introduced and analyzed in [29]. In particular, it is proved in [29] that the global solvability in $\mathrm{L}^{2}$-spaces holds true for the 3-dimensional case. More recently, in [31] a finite volume method was adopted to solve the coupling of the time-dependent Brinkman-Forchheimer and double-diffusion equations. The focus of this work was on the validity of the Brinkman-Forchheimer model when various combinations of the thermal Rayleigh number, permeability ratio, inclination angle, thermal conductivity and buoyancy ratio are considered. This study allowed the evaluation of the control parameters effect on the flow structure, and heat and mass transfer. Meanwhile, an augmented fully-mixed formulation based on the introduction of the fluid pseudostress tensor, and the pseudoheat and pseudodiffusive vectors (besides the velocity, temperature and concentration fields) was analyzed in [14]. In there, the well-posedness of the problem is achieved by combining a fixed-point strategy, the Lax-Milgram and Banach-Nečas-Babuška theorems, and the well-known Schauder and Banach fixed-point theorems. The corresponding numerical scheme is based on Raviart-Thomas spaces of order $k \geq 0$ for approximating the pseudostress tensor, as well as the pseudoheat and pseudodiffusive vectors, whereas continuous piecewise polynomials of degree $k+1$ are employed for the velocity, and piecewise polynomials of degree $k$ for the temperature and concentration fields. Optimal a priori error estimates were also derived.

We point out that the augmented formulation introduced in [14], and the consequent use of classical RaviartThomas spaces and continuous piecewise polynomials to define the discrete scheme, are originated by the wish of performing the respective solvability analysis of the Brinkman-Forchheimer equations within a Hilbertian framework. However, it is well known that the introduction of additional terms into the formulation, while having some advantages, also leads to much more expensive schemes in terms of complexity and computational implementation. In order to overcome this, in recent years there has arisen an increasing development on Banach spaces-based mixed finite element methods to solve a wide family of single and coupled nonlinear problems in continuum mechanics (see, e.g. $[4,5,10-12,15,18,19])$. This kind of procedures shows two advantages at least: no augmentation is required, and the spaces to which the unknowns belong are the natural ones arising from the application of the Cauchy-Schwarz and Hölder inequalities to the terms resulting from the testing and integration by parts of the equations of the model. As a consequence, simpler and closer to the original physical model formulations are obtained.

According to the above bibliographic discussion, the goal of the present paper is to continue extending the applicability of the aforementioned Banach spaces framework by proposing now a new fully-mixed formulation, without any augmentation procedure, for the coupled problem studied in [14,28]. To this end, we proceed as in [18] and introduce the velocity gradient and pseudostress tensors as auxiliary unknowns, and subsequently eliminate the pressure unknown using the incompressibility condition. In turn, we follow $[18,19]$ and adopt a dualmixed formulation for the double-difussion equations making use of the temperature/concentration gradients and Bernoulli-type vectors as further unknowns. Then, similarly to $[17,18]$, we combine a fixed-point argument, classical results on nonlinear monotone operators, Babuška-Brezzi's theory in Banach spaces, sufficiently small 
data assumptions, and the well known Banach fixed-point theorem, to establish existence and uniqueness of solution of both the continuous and discrete formulations. In this regard, and since the formulation for the double-diffusion equations is similar to the ones employed in $[18,19]$, our present analysis certainly makes use of the corresponding results available there. In addition, applying an ad-hoc Strang-type lemma in Banach spaces, we are able to derive the corresponding a priori error estimates. Next, employing Raviart-Thomas spaces of order $k \geq 0$ for approximating the pseudostress tensor and Bernoulli vectors, and discontinuous piecewise polynomials of degree $k$ for the velocity, temperature, concentration and its corresponding gradients fields, we prove that the method is convergent with optimal rate.

This work is organized as follows. The remainder of this section describes standard notation and functional spaces to be employed throughout the paper. In Section 2, we introduce the model problem and derive the fully-mixed variational formulation in Banach spaces. Next, in Section 3 we establish the well-posedness of this continuous scheme by means of a fixed-point strategy and Banach's fixed-point theorem. The corresponding Galerkin system is introduced and analyzed in Section 4, where the discrete analogue of the theory used in the continuous case is employed to prove existence and uniqueness of solution. In Section 5, an ad-hoc Strang-type lemma in Banach spaces is utilized to derive the corresponding a priori error estimate and the consequent rates of convergence. Finally, the performance of the method is illustrated in Section 6 with several numerical examples in $2 \mathrm{D}$ and $3 \mathrm{D}$, which confirm the aforementioned rates.

\section{Preliminary notations}

Let $\Omega \subset \mathrm{R}^{n}, n \in\{2,3\}$, be a bounded domain with polyhedral boundary $\Gamma$, and let $\mathbf{n}$ be the outward unit normal vector on $\Gamma$. Standard notation will be adopted for Lebesgue spaces $\mathrm{L}^{p}(\Omega)$ and Sobolev spaces $\mathrm{W}^{s, p}(\Omega)$, with $s \in \mathrm{R}$ and $p>1$, whose corresponding norms, either for the scalar, vectorial, or tensorial case, are denoted by $\|\cdot\|_{0, p ; \Omega}$ and $\|\cdot\|_{s, p ; \Omega}$, respectively. In particular, given a non-negative integer $m, \mathrm{~W}^{m, 2}(\Omega)$ is also denoted by $\mathrm{H}^{m}(\Omega)$, and the notations of its norm and seminorm are simplified to $\|\cdot\|_{m, \Omega}$ and $|\cdot|_{m, \Omega}$, respectively. By $\mathbf{M}$ and $\mathbb{M}$ we will denote the corresponding vectorial and tensorial counterparts of the generic scalar functional space $\mathrm{M}$, and $\|\cdot\|$, with no subscripts, will stand for the natural norm of either an element or an operator in any product functional space. In turn, for any vector field $\mathbf{v}=\left(v_{i}\right)_{i=1, n}$, we let $\nabla \mathbf{v}$ and $\operatorname{div}(\mathbf{v})$ be its gradient and divergence, respectively. Furthermore, for any tensor fields $\boldsymbol{\tau}=\left(\tau_{i j}\right)_{i, j=1, n}$ and $\boldsymbol{\zeta}=\left(\zeta_{i j}\right)_{i, j=1, n}$, we let $\operatorname{div}(\boldsymbol{\tau})$ be the divergence operator div acting along the rows of $\boldsymbol{\tau}$, and define the transpose, the trace, the tensor inner product, and the deviatoric tensor, respectively, as

$$
\boldsymbol{\tau}^{\mathrm{t}}:=\left(\tau_{j i}\right)_{i, j=1, n}, \quad \operatorname{tr}(\boldsymbol{\tau}):=\sum_{i=1}^{n} \tau_{i i}, \quad \boldsymbol{\tau}: \boldsymbol{\zeta}:=\sum_{i, j=1}^{n} \tau_{i j} \zeta_{i j}, \quad \text { and } \quad \boldsymbol{\tau}^{\mathrm{d}}:=\boldsymbol{\tau}-\frac{1}{n} \operatorname{tr}(\boldsymbol{\tau}) \mathbb{I},
$$

where $\mathbb{I}$ is the identity matrix in $\mathbb{R}:=\mathrm{R}^{n \times n}$. In what follows, when no confusion arises, $|\cdot|$ will denote the Euclidean norm in $\mathbf{R}:=\mathrm{R}^{n}$ or $\mathbb{R}$. Additionally, we recall that

$$
\mathbb{H}(\operatorname{div} ; \Omega):=\left\{\boldsymbol{\tau} \in \mathbb{L}^{2}(\Omega): \operatorname{div}(\boldsymbol{\tau}) \in \mathbf{L}^{2}(\Omega)\right\},
$$

equipped with the usual norm $\|\boldsymbol{\tau}\|_{\mathbf{d i v} ; \Omega}^{2}:=\|\boldsymbol{\tau}\|_{0, \Omega}^{2}+\|\operatorname{div}(\boldsymbol{\tau})\|_{0, \Omega}^{2}$, is a standard Hilbert space in the realm of mixed problems. In addition, $\mathrm{H}^{1 / 2}(\Gamma)$ is the space of traces of functions of $\mathrm{H}^{1}(\Omega)$ and $\mathrm{H}^{-1 / 2}(\Gamma)$ denotes its dual. Also, by $\langle\cdot, \cdot\rangle_{\Gamma}$ we will denote the corresponding product of duality between $\mathrm{H}^{-1 / 2}(\Gamma)$ and $\mathrm{H}^{1 / 2}(\Gamma)$ (and also between $\mathbf{H}^{-1 / 2}(\Gamma)$ and $\left.\mathbf{H}^{1 / 2}(\Gamma)\right)$.

\section{The COntinuous Formulation}

In this section we introduce the model problem and derive the corresponding weak formulation. 


\subsection{The model problem}

In what follows we consider the model introduced in [28] (see also [14], Sect. 2), which is given by a steady double-diffusive convection system in a fluid saturated porous medium. More precisely, we focus on solving the coupling of the incompressible Brinkman-Forchheimer and double-diffusion equations, which reduces to finding a velocity field $\mathbf{u}$, a pressure field $p$, a temperature field $\phi_{1}$ and a concentration field $\phi_{2}$, the latter two defining a vector $\phi:=\left(\phi_{1}, \phi_{2}\right)$, such that

$$
\begin{aligned}
-\nu \Delta \mathbf{u}+\mathbf{K}^{-1} \mathbf{u}+\mathrm{F}|\mathbf{u}| \mathbf{u}+\nabla p & =\mathbf{f}(\boldsymbol{\phi}) & & \text { in } \Omega, \\
\operatorname{div}(\mathbf{u}) & =0 & & \text { in } \Omega, \\
-\operatorname{div}\left(\mathbf{Q}_{1} \nabla \phi_{1}\right)+\mathrm{R}_{1} \mathbf{u} \cdot \nabla \phi_{1} & =0 & & \text { in } \Omega, \\
-\operatorname{div}\left(\mathbf{Q}_{2} \nabla \phi_{2}\right)+\mathrm{R}_{2} \mathbf{u} \cdot \nabla \phi_{2} & =0 & & \text { in } \Omega,
\end{aligned}
$$

with parameters $\nu:=\mathrm{D}_{a} \widetilde{\mu} / \mu$ and $\mathrm{F}:=\vartheta \mathrm{D}_{a} \mathrm{R}_{1}$, where $\mathrm{D}_{a}$ stands for the Darcy number, $\widetilde{\mu}$ the viscosity, $\mu$ the effective viscosity, $R_{1}$ the thermal Rayleigh number, $R_{2}$ the solute Rayleigh number, and $\vartheta$ is a real number that can be calculated experimentally. In addition, the external force $\mathbf{f}$ is defined by

$$
\mathbf{f}(\phi):=-\left(\phi_{1}-\phi_{1, \mathrm{r}}\right) \mathbf{g}+\frac{1}{\varrho}\left(\phi_{2}-\phi_{2, \mathrm{r}}\right) \mathbf{g},
$$

with $\mathbf{g}$ representing the potential type gravitational acceleration, $\phi_{1, \mathrm{r}}$ the reference temperature, $\phi_{2, \mathrm{r}}$ the reference concentration of a solute, and $\varrho$ is another parameter experimentally valued that can be assumed to be $\geq 1$ (see [28], Sect. 2 for details). The spaces to which $\phi_{1, \mathrm{r}}$ and $\phi_{2, \mathrm{r}}$ belong will be specified later on. In turn, the permeability, and the thermal diffusion and concentration diffusion tensors, are denoted by $\mathbf{K}, \mathbf{Q}_{1}$ and $\mathbf{Q}_{2}$, respectively, all them belonging to $\mathbb{L}^{\infty}(\Omega)$. Moreover, the inverse of $\mathbf{K}$ and tensors $\mathbf{Q}_{1}, \mathbf{Q}_{2}$, are uniformly positive definite tensors, which means that there exist positive constants $C_{\mathbf{K}}, C_{\mathbf{Q}_{1}}$, and $C_{\mathbf{Q}_{2}}$, such that

$$
\mathbf{v} \cdot \mathbf{K}^{-1}(\mathbf{x}) \mathbf{v} \geq C_{\mathbf{K}}|\mathbf{v}|^{2} \quad \text { and } \quad \mathbf{v} \cdot \mathbf{Q}_{j}(\mathbf{x}) \mathbf{v} \geq C_{\mathbf{Q}_{j}}|\mathbf{v}|^{2} \quad \forall \mathbf{v} \in \mathrm{R}^{n}, \forall \mathbf{x} \in \Omega, \quad j \in\{1,2\} .
$$

Equations (2.1) are complemented with Dirichlet boundary conditions for the velocity, the temperature, and the concentration fields, that is

$$
\mathbf{u}=\mathbf{u}_{\mathrm{D}}, \quad \phi_{1}=\phi_{1, \mathrm{D}}, \quad \text { and } \quad \phi_{2}=\phi_{2, \mathrm{D}} \quad \text { on } \quad \Gamma,
$$

with given data $\mathbf{u}_{\mathrm{D}} \in \mathbf{H}^{1 / 2}(\Gamma), \phi_{1, \mathrm{D}} \in \mathrm{H}^{1 / 2}(\Gamma)$ and $\phi_{2, \mathrm{D}} \in \mathrm{H}^{1 / 2}(\Gamma)$. Owing to the incompressibility of the fluid and the Dirichlet boundary condition for $\mathbf{u}$, the datum $\mathbf{u}_{\mathrm{D}}$ must satisfy the compatibility condition

$$
\int_{\Gamma} \mathbf{u}_{\mathrm{D}} \cdot \mathbf{n}=0
$$

In addition, due to the first equation of (2.1), and in order to guarantee uniqueness of the pressure, this unknown will be sought in the space

$$
\mathrm{L}_{0}^{2}(\Omega):=\left\{q \in \mathrm{L}^{2}(\Omega): \int_{\Omega} q=0\right\} .
$$

Next, in order to derive a new fully-mixed formulation for (2.1)-(2.5), and unlike [14], we do not employ any augmentation procedure and simply proceed as in [18] (see also [19]). More precisely, we now introduce as further unknowns the velocity gradient $\mathbf{t}$, the pseudostress tensor $\boldsymbol{\sigma}$, the temperature/concentration gradient $\widetilde{\mathbf{t}}_{j}$, and suitable auxiliary variables $\boldsymbol{\rho}_{j}$ depending on $\widetilde{\mathbf{t}}_{j}, \mathbf{u}$, and $\phi_{j}$, all of which are defined, respectively, by

$$
\mathbf{t}:=\nabla \mathbf{u}, \quad \boldsymbol{\sigma}:=\nu \mathbf{t}-p \mathbb{I}, \quad \widetilde{\mathbf{t}}_{j}:=\nabla \phi_{j}, \quad \boldsymbol{\rho}_{j}:=\mathbf{Q}_{j} \widetilde{\mathbf{t}}_{j}-\frac{1}{2} \mathbf{R}_{j} \phi_{j} \mathbf{u}, \quad \forall j \in\{1,2\}, \quad \text { in } \quad \Omega .
$$


In this way, applying the matrix trace to the tensors $\mathbf{t}$ and $\boldsymbol{\sigma}$, and utilizing the incompressibility condition $\operatorname{div}(\mathbf{u})=0$ in $\Omega$, one arrives at $\operatorname{tr}(\mathbf{t})=0$ in $\Omega$ and

$$
p=-\frac{1}{n} \operatorname{tr}(\boldsymbol{\sigma}) \text { in } \Omega .
$$

Hence, replacing back (2.7) in the second equation of (2.6), we find that the model problem (2.1)-(2.4) can be rewritten, equivalently, as follows: Find $(\mathbf{u}, \mathbf{t}, \boldsymbol{\sigma})$ and $\left(\phi_{j}, \widetilde{\mathbf{t}}_{j}, \boldsymbol{\rho}_{j}\right), j \in\{1,2\}$, in suitable spaces to be indicated below, such that

$$
\begin{aligned}
\nabla \mathbf{u} & =\mathbf{t} & & \text { in } \Omega, \\
\nu \mathbf{t} & =\boldsymbol{\sigma}^{\mathrm{d}} & & \text { in } \Omega, \\
\mathbf{K}^{-1} \mathbf{u}+\mathrm{F}|\mathbf{u}| \mathbf{u}-\operatorname{div}(\boldsymbol{\sigma}) & =\mathbf{f}(\boldsymbol{\phi}) & & \text { in } \Omega, \\
\int_{\Omega} \operatorname{tr}(\boldsymbol{\sigma}) & =0, & & \\
\nabla \phi_{j} & =\widetilde{\mathbf{t}}_{j} & & \text { in } \Omega, \\
\mathbf{Q}_{j} \widetilde{\mathbf{t}}_{j}-\frac{1}{2} \mathrm{R}_{j} \phi_{j} \mathbf{u} & =\boldsymbol{\rho}_{j} & & \text { in } \Omega, \\
\frac{1}{2} \mathrm{R}_{j} \mathbf{u} \cdot \widetilde{\mathbf{t}}_{j}-\operatorname{div}\left(\boldsymbol{\rho}_{j}\right) & =0 & & \text { in } \Omega, \\
\mathbf{u}=\mathbf{u}_{\mathrm{D}} \text { and } \quad \boldsymbol{\phi} & =\phi_{\mathrm{D}} & & \text { on } \Gamma,
\end{aligned}
$$

where the Dirichlet datum for $\phi$ is certainly given by $\phi_{\mathrm{D}}:=\left(\phi_{1, \mathrm{D}}, \phi_{2, \mathrm{D}}\right)$. At this point we stress that, as suggested by (2.7), $p$ is eliminated from the present formulation and computed afterwards in terms of $\boldsymbol{\sigma}$ by using that identity. This fact justifies the fourth equation in (2.8), which aims to ensure that the resulting $p$ does belong to $\mathrm{L}_{0}^{2}(\Omega)$.

\subsection{The fully-mixed variational formulation}

In this section we follow $[18,19]$ to derive a fully-mixed formulation in a Banach spaces framework for the coupled system given by (2.8). To this end, we first multiply the third equation of (2.8) by a test function $\mathbf{v}$ associated with the unknown $\mathbf{u}$, which formally yields

$$
\int_{\Omega} \mathbf{K}^{-1} \mathbf{u} \cdot \mathbf{v}+\mathbf{F} \int_{\Omega}|\mathbf{u}| \mathbf{u} \cdot \mathbf{v}-\int_{\Omega} \mathbf{v} \cdot \operatorname{div}(\sigma)=\int_{\Omega} \mathbf{f}(\phi) \cdot \mathbf{v} .
$$

Then, applying the Hölder and Cauchy-Schwarz inequalities, we find that the Forchheimer term, given by the second expression in (2.9), can be bounded as

$$
\left|\int_{\Omega}\right| \mathbf{u}|\mathbf{u} \cdot \mathbf{v}| \leq\|\mathbf{u}\|_{0,2 \ell ; \Omega}\|\mathbf{u}\|_{0,2 \ell ; \Omega}\|\mathbf{v}\|_{0, j ; \Omega}
$$

where $\ell, j \in(1,+\infty)$ are conjugate to each other, that is $\frac{1}{\ell}+\frac{1}{j}=1$. In this way, while we could continue our analysis with arbitrary values of $\ell$ and $j$, and hence with $\mathbf{u}$ and $\mathbf{v}$ belonging to the Lebesgue spaces $\mathbf{L}^{2 \ell}(\Omega)$ and $\mathbf{L}^{j}(\Omega)$, respectively, we prefer for simplicity to make the latter to coincide, that is such that $2 \ell=j$, which gives $\ell=\frac{3}{2}$ and $j=3$, so that both $\mathbf{u}$ and $\mathbf{v}$ belong to $\mathbf{L}^{3}(\Omega)$. Consequently, the fact that $\mathbf{L}^{3}(\Omega)$ is certainly contained in $\mathbf{L}^{2}(\Omega)$ and the uniform boundedness of $\mathbf{K}$ guarantee that the first term in (2.9) is bounded as well, whereas for the third and fourth ones to be well-defined we need to impose that $\operatorname{div}(\boldsymbol{\sigma})$ and $\mathbf{f}(\boldsymbol{\phi})$ lie in $\mathbf{L}^{3 / 2}(\Omega)$.

Now, given $t \in(1,+\infty)$, we introduce the Banach space

$$
\mathbb{H}\left(\operatorname{div}_{t} ; \Omega\right):=\left\{\boldsymbol{\tau} \in \mathbb{L}^{2}(\Omega): \operatorname{div}(\boldsymbol{\tau}) \in \mathbf{L}^{t}(\Omega)\right\},
$$


which is endowed with the natural norm

$$
\|\boldsymbol{\tau}\|_{\operatorname{div}_{t} ; \Omega}:=\|\boldsymbol{\tau}\|_{0, \Omega}+\|\operatorname{div}(\boldsymbol{\tau})\|_{0, t ; \Omega} \quad \forall \boldsymbol{\tau} \in \mathbb{H}\left(\operatorname{div}_{t} ; \Omega\right)
$$

Then, proceeding as in equation (1.43), Section 1.3.4 of [24] (see also [10], Sect. 4.1, [18], Sect. 3.1), one can prove that for each $t \geq \frac{2 n}{n+2}$ there holds

$$
\langle\boldsymbol{\tau} \mathbf{n}, \mathbf{v}\rangle_{\Gamma}=\int_{\Omega}\{\boldsymbol{\tau}: \nabla \mathbf{v}+\mathbf{v} \cdot \operatorname{div}(\boldsymbol{\tau})\} \quad \forall(\boldsymbol{\tau}, \mathbf{v}) \in \mathbb{H}\left(\operatorname{div}_{t} ; \Omega\right) \times \mathbf{H}^{1}(\Omega),
$$

which says, in particular, that $\boldsymbol{\tau} \mathbf{n} \in \mathbf{H}^{-1 / 2}(\Gamma)$ for all $\boldsymbol{\tau} \in \mathbb{H}\left(\operatorname{div}_{t} ; \Omega\right)$. In turn, we stress that the fact that $\mathrm{L}^{2}(\Omega)$ is continuously embedded into $\mathrm{L}^{t}(\Omega)$ for each $t \in(1,2)$ implies that for this range of $t$ there holds $\mathbb{H}(\operatorname{div} ; \Omega) \subset \mathbb{H}\left(\operatorname{div}_{t} ; \Omega\right)$.

Next, if we look originally for $\mathbf{t}$ in $\mathbb{L}^{2}(\Omega)$, then from the first equation of (2.8) we would have that $\mathbf{u} \in \mathbf{H}^{1}(\Omega)$, which is embedded in $\mathbf{L}^{3}(\Omega)$, so that applying (2.11) to $\boldsymbol{\tau} \in \mathbb{H}\left(\mathbf{d i v}_{3 / 2} ; \Omega\right)$ and $\mathbf{u}$, and employing the Dirichlet boundary condition on $\mathbf{u}$, we obtain from that equation that

$$
\int_{\Omega} \boldsymbol{\tau}: \mathbf{t}+\int_{\Omega} \mathbf{u} \cdot \operatorname{div}(\boldsymbol{\tau})=\left\langle\boldsymbol{\tau} \mathbf{n}, \mathbf{u}_{\mathrm{D}}\right\rangle_{\Gamma}
$$

Actually, because of the incompressibility condition satisfied by $\mathbf{u}$ ( $c f$. second equation of (2.1)), which is reconfirmed by the second equation of (2.8), $\mathbf{t}$ must be sought in $\mathbb{L}_{\mathrm{tr}}^{2}(\Omega)$, where

$$
\mathbb{L}_{\mathrm{tr}}^{2}(\Omega):=\left\{\mathbf{r} \in \mathbb{L}^{2}(\Omega): \operatorname{tr}(\mathbf{r})=0 \quad \text { in } \quad \Omega\right\}
$$

Moreover, testing the aforementioned last identity against $\mathbf{r} \in \mathbb{L}_{\mathrm{tr}}^{2}(\Omega)$, which requires $\sigma \in \mathbb{L}^{2}(\Omega)$, thus yielding $\boldsymbol{\sigma} \in \mathbb{H}\left(\operatorname{div}_{3 / 2} ; \Omega\right)$ as well (recall that $\operatorname{div}(\boldsymbol{\sigma})$ must lie in $\mathbf{L}^{3 / 2}(\Omega)$ ), we arrive at

$$
\nu \int_{\Omega} \mathbf{t}: \mathbf{r}-\int_{\Omega} \boldsymbol{\sigma}^{\mathrm{d}}: \mathbf{r}=0
$$

According to the previous analysis, the weak formulation of the Brinkman-Forchheimer part of the coupled problem (2.8) reduces at first instance to: Find $(\mathbf{u}, \mathbf{t}, \boldsymbol{\sigma}) \in \mathbf{L}^{3}(\Omega) \times \mathbb{L}_{\mathrm{tr}}^{2}(\Omega) \times \mathbb{H}\left(\mathbf{d i v}_{3 / 2} ; \Omega\right)$ such that (2.9), (2.12), and (2.13) hold for all $(\mathbf{v}, \mathbf{r}, \boldsymbol{\tau}) \in \mathbf{L}^{3}(\Omega) \times \mathbb{L}_{\mathrm{tr}}^{2}(\Omega) \times \mathbb{H}\left(\operatorname{div}_{3 / 2} ; \Omega\right)$.

However, similarly as in $[18]$ (see also $[10,19]$ ), we consider the decomposition

$$
\mathbb{H}\left(\operatorname{div}_{3 / 2} ; \Omega\right)=\mathbb{H}_{0}\left(\operatorname{div}_{3 / 2} ; \Omega\right) \oplus \mathrm{RI},
$$

where

$$
\mathbb{H}_{0}\left(\operatorname{div}_{3 / 2} ; \Omega\right):=\left\{\boldsymbol{\tau} \in \mathbb{H}\left(\operatorname{div}_{3 / 2} ; \Omega\right): \int_{\Omega} \operatorname{tr}(\boldsymbol{\tau})=0\right\}
$$

and $\mathrm{RI}$ is a topological supplement for $\mathbb{H}_{0}\left(\operatorname{div}_{3 / 2} ; \Omega\right)$.

Then, it is clear from the fourth equation of (2.8) that actually $\boldsymbol{\sigma} \in \mathbb{H}_{0}\left(\mathbf{d i v}_{3 / 2} ; \Omega\right)$. In addition, it is readily seen, using the compatibility condition (2.5), that both sides of (2.12) explicitly vanish when $\boldsymbol{\tau} \in \mathrm{RI}$, and hence testing against $\boldsymbol{\tau} \in \mathbb{H}\left(\mathbf{d i v}_{3 / 2} ; \Omega\right)$ is equivalent to doing it against $\boldsymbol{\tau} \in \mathbb{H}_{0}\left(\mathbf{d i v}_{3 / 2} ; \Omega\right)$. Therefore, denoting from now on

$$
\overrightarrow{\mathbf{u}}:=(\mathbf{u}, \mathbf{t}), \overrightarrow{\mathbf{w}}:=(\mathbf{w}, \mathbf{s}), \overrightarrow{\mathbf{v}}:=(\mathbf{v}, \mathbf{r}) \in \mathbf{L}^{3}(\Omega) \times \mathbb{L}_{\mathrm{tr}}^{2}(\Omega),
$$

and suitably grouping the equations (2.9), (2.12), and (2.13), the aforementioned weak formulation reads: Find $(\overrightarrow{\mathbf{u}}, \boldsymbol{\sigma}) \in\left(\mathbf{L}^{3}(\Omega) \times \mathbb{L}_{\mathrm{tr}}^{2}(\Omega)\right) \times \mathbb{H}_{0}\left(\operatorname{div}_{3 / 2} ; \Omega\right)$ such that

$$
[a(\overrightarrow{\mathbf{u}}), \overrightarrow{\mathbf{v}}]+[b(\overrightarrow{\mathbf{v}}), \boldsymbol{\sigma}]=\left[F_{\boldsymbol{\phi}}, \overrightarrow{\mathbf{v}}\right] \quad \forall \overrightarrow{\mathbf{v}} \in \mathbf{L}^{3}(\Omega) \times \mathbb{L}_{\mathrm{tr}}^{2}(\Omega),
$$




$$
[b(\overrightarrow{\mathbf{u}}), \boldsymbol{\tau}]=\left[G_{\mathrm{D}}, \boldsymbol{\tau}\right] \quad \forall \boldsymbol{\tau} \in \mathbb{H}_{0}\left(\operatorname{div}_{3 / 2} ; \Omega\right),
$$

where the nonlinear operator $a:\left(\mathbf{L}^{3}(\Omega) \times \mathbb{L}_{\mathrm{tr}}^{2}(\Omega)\right) \rightarrow\left(\mathbf{L}^{3}(\Omega) \times \mathbb{L}_{\mathrm{tr}}^{2}(\Omega)\right)^{\prime}$, the linear and bounded operator $b:\left(\mathbf{L}^{3}(\Omega) \times \mathbb{L}_{\mathrm{tr}}^{2}(\Omega)\right) \rightarrow \mathbb{H}_{0}\left(\operatorname{div}_{3 / 2} ; \Omega\right)^{\prime}$, and the functional $G_{\mathrm{D}} \in \mathbb{H}_{0}\left(\operatorname{div}_{3 / 2} ; \Omega\right)^{\prime}$, are defined, respectively, as

$$
\begin{aligned}
{[a(\overrightarrow{\mathbf{w}}), \overrightarrow{\mathbf{v}}]: } & =\int_{\Omega} \mathbf{K}^{-1} \mathbf{w} \cdot \mathbf{v}+\mathrm{F} \int_{\Omega}|\mathbf{w}| \mathbf{w} \cdot \mathbf{v}+\nu \int_{\Omega} \mathbf{s}: \mathbf{r}, \\
{[b(\overrightarrow{\mathbf{v}}), \boldsymbol{\tau}] } & :=-\int_{\Omega} \mathbf{v} \cdot \operatorname{div}(\boldsymbol{\tau})-\int_{\Omega} \boldsymbol{\tau}: \mathbf{r},
\end{aligned}
$$

and

$$
\left[G_{\mathrm{D}}, \boldsymbol{\tau}\right]:=-\left\langle\boldsymbol{\tau} \mathbf{n}, \mathbf{u}_{\mathrm{D}}\right\rangle_{\Gamma},
$$

for all $\overrightarrow{\mathbf{w}}, \overrightarrow{\mathbf{v}} \in \mathbf{L}^{3}(\Omega) \times \mathbb{L}_{\mathrm{tr}}^{2}(\Omega)$, and for all $\boldsymbol{\tau} \in \mathbb{H}_{0}\left(\operatorname{div}_{3 / 2} ; \Omega\right)$. In turn, given $\varphi:=\left(\varphi_{1}, \varphi_{2}\right)$ in the spaces to be indicated below, the functional $F_{\varphi}$ is given by

$$
\left[F_{\boldsymbol{\varphi}}, \overrightarrow{\mathbf{v}}\right]:=\int_{\Omega} \mathbf{f}(\boldsymbol{\varphi}) \cdot \mathbf{v} \quad \forall \overrightarrow{\mathbf{v}} \in \mathbf{L}^{3}(\Omega) \times \mathbb{L}_{\mathrm{tr}}^{2}(\Omega) .
$$

In all the terms above, $[\cdot, \cdot]$ denotes the duality pairing induced by the corresponding operators.

On the other hand, for the double-diffusion equations in (2.8) we proceed analogously as for the derivation of (2.9), (2.12), and (2.13). In fact, multiplying the sixth equation of (2.8) by a test function $\widetilde{\mathbf{r}}_{j}$ associated with the unknown $\widetilde{\mathbf{t}}_{j}$, we formally obtain

$$
\int_{\Omega} \mathbf{Q}_{j} \widetilde{\mathbf{t}}_{j} \cdot \widetilde{\mathbf{r}}_{j}-\frac{1}{2} \mathrm{R}_{j} \int_{\Omega} \phi_{j} \mathbf{u} \cdot \widetilde{\mathbf{r}}_{j}-\int_{\Omega} \boldsymbol{\rho}_{j} \cdot \widetilde{\mathbf{r}}_{j}=0 .
$$

Then, employing again the Cauchy-Schwarz and Hölder inequalities, we find that the convective term from the foregoing equation can be bounded as

$$
\left|\int_{\Omega} \phi_{j} \mathbf{u} \cdot \widetilde{\mathbf{r}}_{j}\right| \leq\left\|\phi_{j}\right\|_{0,2 r ; \Omega}\|\mathbf{u}\|_{0,2 s ; \Omega}\left\|\widetilde{\mathbf{r}}_{j}\right\|_{0, \Omega}
$$

where $r$ and $s$ are conjugate to each other. But, knowing already that $\mathbf{u}$ is sought in $\mathbf{L}^{3}(\Omega)$, we are forced to choose $s=3 / 2$, which yields $r=3$, and hence we look for $\phi_{j}$ in $\mathrm{L}^{6}(\Omega)$, whereas $\widetilde{\mathbf{r}}_{j}$ lies in $\mathbf{L}^{2}(\Omega)$. As a consequence of the latter and the fact that $\mathbf{Q}_{j} \in \mathbb{L}^{\infty}(\Omega), j \in\{1,2\}$, we notice that the first and third terms of (2.19) are bounded if we look for both $\widetilde{\mathbf{t}}_{j}$ and $\boldsymbol{\rho}_{j}$ in $\mathbf{L}^{2}(\Omega)$. Now, we introduce the vector version of (2.10), that is for each $t \in(1,+\infty)$ we set

$$
\mathbf{H}\left(\operatorname{div}_{t} ; \Omega\right):=\left\{\boldsymbol{\eta} \in \mathbf{L}^{2}(\Omega): \operatorname{div}(\boldsymbol{\eta}) \in \mathrm{L}^{t}(\Omega)\right\} .
$$

Then, noting from the fifth equation of (2.8) that $\phi_{j} \in \mathrm{H}^{1}(\Omega)$, which is embedded in $\mathrm{L}^{6}(\Omega)$, and then applying the vector-scalar version of $(2.11)$ to $\boldsymbol{\eta}_{j} \in \mathbf{H}\left(\operatorname{div}_{6 / 5} ; \Omega\right)$ and $\phi_{j}$, and using the Dirichlet boundary condition on $\phi_{j}$, it follows from that equation that

$$
\int_{\Omega} \widetilde{\mathbf{t}}_{j} \cdot \boldsymbol{\eta}_{j}+\int_{\Omega} \phi_{j} \operatorname{div}\left(\boldsymbol{\eta}_{j}\right)=\left\langle\boldsymbol{\eta}_{j} \cdot \mathbf{n}, \phi_{j, \mathrm{D}}\right\rangle_{\Gamma}
$$

Finally, testing the seventh equation of (2.8) against $\psi_{j} \in \mathrm{L}^{6}(\Omega)$, which requires $\operatorname{div}\left(\boldsymbol{\rho}_{j}\right) \in \mathrm{L}^{6 / 5}(\Omega)$, thus yielding $\boldsymbol{\rho}_{j} \in \mathbf{H}\left(\operatorname{div}_{6 / 5} ; \Omega\right)$ as well, we get

$$
\frac{1}{2} \mathrm{R}_{j} \int_{\Omega} \psi_{j} \mathbf{u} \cdot \widetilde{\mathbf{t}}_{j}-\int_{\Omega} \psi_{j} \operatorname{div}\left(\boldsymbol{\rho}_{j}\right)=0
$$


Similarly as before for $\mathbb{H}\left(\operatorname{div}_{t} ; \Omega\right)$, we notice here that $\boldsymbol{\eta} \cdot \mathbf{n} \in \mathrm{H}^{-1 / 2}(\Gamma)$ for all $\boldsymbol{\eta} \in \mathbf{H}\left(\operatorname{div}_{t} ; \Omega\right), t \in(1,+\infty)$. In addition, for each $t \in(1,2)$ there holds $\mathbf{H}(\operatorname{div} ; \Omega) \subset \mathbf{H}\left(\operatorname{div}_{t} ; \Omega\right)$.

Hence, setting from now on

$$
\vec{\phi}_{j}:=\left(\phi_{j}, \widetilde{\mathbf{t}}_{j}\right), \vec{\varphi}_{j}:=\left(\varphi_{j}, \widetilde{\mathbf{s}}_{j}\right), \vec{\psi}_{j}:=\left(\psi_{j}, \widetilde{\mathbf{r}}_{j}\right) \in \mathrm{L}^{6}(\Omega) \times \mathbf{L}^{2}(\Omega)
$$

and conveniently grouping (2.19)-(2.21), the weak formulation of the double-diffusion equations in (2.8) reads: Find $\left(\vec{\phi}_{j}, \boldsymbol{\rho}_{j}\right) \in\left(\mathrm{L}^{6}(\Omega) \times \mathbf{L}^{2}(\Omega)\right) \times \mathbf{H}\left(\operatorname{div}_{6 / 5} ; \Omega\right), j \in\{1,2\}$, such that

$$
\begin{aligned}
{\left[\widetilde{a}_{j}\left(\vec{\phi}_{j}\right), \vec{\psi}_{j}\right]+\left[c_{j}(\mathbf{u})\left(\vec{\phi}_{j}\right), \vec{\psi}_{j}\right]+\left[\widetilde{b}\left(\vec{\psi}_{j}\right), \boldsymbol{\rho}_{j}\right] } & =0 & & \forall \vec{\psi}_{j} \in \mathrm{L}^{6}(\Omega) \times \mathbf{L}^{2}(\Omega), \\
{\left[\widetilde{b}\left(\vec{\phi}_{j}\right), \boldsymbol{\eta}_{j}\right] } & =\left[\widetilde{G}_{j}, \boldsymbol{\eta}_{j}\right] & & \forall \boldsymbol{\eta}_{j} \in \mathbf{H}\left(\operatorname{div}_{6 / 5} ; \Omega\right),
\end{aligned}
$$

where the linear and bounded operators $\widetilde{a}_{j}, c_{j}(\mathbf{w}):\left(\mathrm{L}^{6}(\Omega) \times \mathbf{L}^{2}(\Omega)\right) \rightarrow\left(\mathrm{L}^{6}(\Omega) \times \mathbf{L}^{2}(\Omega)\right)^{\prime}$ (for a given $\mathbf{w} \in$ $\left.\mathbf{L}^{3}(\Omega)\right)$, and $\widetilde{b}:\left(\mathrm{L}^{6}(\Omega) \times \mathbf{L}^{2}(\Omega)\right) \rightarrow \mathbf{H}\left(\operatorname{div}_{6 / 5} ; \Omega\right)^{\prime}$, and the bounded linear functional $\widetilde{G}_{j} \in \mathbf{H}\left(\operatorname{div}_{6 / 5} ; \Omega\right)^{\prime}$, are defined, respectively, as

$$
\begin{aligned}
{\left[\widetilde{a}_{j}\left(\vec{\varphi}_{j}\right), \vec{\psi}_{j}\right] } & :=\int_{\Omega} \mathbf{Q}_{j} \widetilde{\mathbf{s}}_{j} \cdot \widetilde{\mathbf{r}}_{j}, \\
{\left[c_{j}(\mathbf{w})\left(\vec{\varphi}_{j}\right), \vec{\psi}_{j}\right] } & :=\frac{1}{2} \mathrm{R}_{j}\left\{\int_{\Omega} \psi_{j} \mathbf{w} \cdot \widetilde{\mathbf{s}}_{j}-\int_{\Omega} \varphi_{j} \mathbf{w} \cdot \widetilde{\mathbf{r}}_{j}\right\}, \\
{\left[\widetilde{b}\left(\vec{\psi}_{j}\right), \boldsymbol{\eta}_{j}\right] } & :=-\int_{\Omega} \psi_{j} \operatorname{div}\left(\boldsymbol{\eta}_{j}\right)-\int_{\Omega} \boldsymbol{\eta}_{j} \cdot \widetilde{\mathbf{r}}_{j},
\end{aligned}
$$

and

$$
\left[\widetilde{G}_{j}, \boldsymbol{\eta}_{j}\right]:=-\left\langle\boldsymbol{\eta}_{j} \cdot \mathbf{n}, \phi_{j, \mathrm{D}}\right\rangle_{\Gamma},
$$

for all $\vec{\varphi}_{j}, \vec{\psi}_{j} \in \mathrm{L}^{6}(\Omega) \times \mathbf{L}^{2}(\Omega)$, and for all $\boldsymbol{\eta}_{j} \in \mathbf{H}\left(\operatorname{div}_{6 / 5} ; \Omega\right)$.

Summarizing, the fully-mixed formulation for the coupled problem (2.8) reduces to (2.14) and (2.22), that is: Find $(\overrightarrow{\mathbf{u}}, \boldsymbol{\sigma}) \in\left(\mathbf{L}^{3}(\Omega) \times \mathbb{L}_{\mathrm{tr}}^{2}(\Omega)\right) \times \mathbb{H}_{0}\left(\operatorname{div}_{3 / 2} ; \Omega\right)$ and $\left(\vec{\phi}_{j}, \boldsymbol{\rho}_{j}\right) \in\left(\mathrm{L}^{6}(\Omega) \times \mathbf{L}^{2}(\Omega)\right) \times \mathbf{H}\left(\operatorname{div}_{6 / 5} ; \Omega\right), j \in\{1,2\}$, such that

$$
\begin{aligned}
{[a(\overrightarrow{\mathbf{u}}), \overrightarrow{\mathbf{v}}]+[b(\overrightarrow{\mathbf{v}}), \boldsymbol{\sigma}] } & =\left[F_{\boldsymbol{\phi}}, \overrightarrow{\mathbf{v}}\right] & & \forall \overrightarrow{\mathbf{v}} \in \mathbf{L}^{3}(\Omega) \times \mathbb{L}_{\mathrm{tr}}^{2}(\Omega), \\
{[b(\overrightarrow{\mathbf{u}}), \boldsymbol{\tau}] } & =\left[G_{\mathrm{D}}, \boldsymbol{\tau}\right] & & \forall \boldsymbol{\tau} \in \mathbb{H}_{0}\left(\operatorname{div}_{3 / 2} ; \Omega\right), \\
{\left[\widetilde{a}_{j}\left(\vec{\phi}_{j}\right), \vec{\psi}_{j}\right]+\left[c_{j}(\mathbf{u})\left(\vec{\phi}_{j}\right), \vec{\psi}_{j}\right]+\left[\widetilde{b}\left(\vec{\psi}_{j}\right), \boldsymbol{\rho}_{j}\right] } & =0 & & \forall \vec{\psi}_{j} \in \mathrm{L}^{6}(\Omega) \times \mathbf{L}^{2}(\Omega), \\
{\left[\widetilde{b}\left(\vec{\phi}_{j}\right), \boldsymbol{\eta}_{j}\right] } & =\left[\widetilde{G}_{j}, \boldsymbol{\eta}_{j}\right] & & \forall \boldsymbol{\eta}_{j} \in \mathbf{H}\left(\operatorname{div}_{6 / 5} ; \Omega\right),
\end{aligned}
$$

\section{Analysis of the COUPLED PROBlem}

In this section we combine classical results on nonlinear monotone operators and the Babuška-Brezzi theory in Banach spaces, with the Banach fixed-point theorem, to prove the well-posedness of (2.27) under suitable smallness assumptions on the data. To that end we first collect some previous results and notations that will serve for the forthcoming analysis.

\subsection{Preliminaries}

We begin by establishing the following abstract result.

Theorem 3.1. Let $X_{1}, X_{2}$ and $Y$ be separable and reflexive Banach spaces, being $X_{1}$ and $X_{2}$ uniformly convex, and set $X:=X_{1} \times X_{2}$. Let $\mathcal{A}: X \rightarrow X^{\prime}$ be a nonlinear operator, $\mathcal{B} \in \mathcal{L}\left(X, Y^{\prime}\right)$, and let $V$ be the kernel of $\mathcal{B}$, that is,

$$
V:=\left\{\vec{v}=\left(v_{1}, v_{2}\right) \in X: \mathcal{B}(\vec{v})=\mathbf{0}\right\}
$$




\section{Assume that}

(i) there exist constants $L>0$ and $p_{1}, p_{2} \geq 2$, such that

$$
\|\mathcal{A}(\vec{u})-\mathcal{A}(\vec{v})\|_{X^{\prime}} \leq L \sum_{j=1}^{2}\left\{\left\|u_{j}-v_{j}\right\|_{X_{j}}+\left(\left\|u_{j}\right\|_{X_{j}}+\left\|v_{j}\right\|_{X_{j}}\right)^{p_{j}-2}\left\|u_{j}-v_{j}\right\|_{X_{j}}\right\}
$$

for all $\vec{u}=\left(u_{1}, u_{2}\right), \vec{v}=\left(v_{1}, v_{2}\right) \in X$,

(ii) the family of operators $\left\{\mathcal{A}(\cdot+\vec{z}): V \rightarrow V^{\prime}: \vec{z} \in X\right\}$ is uniformly strongly monotone, that is there exists $\alpha>0$ such that

$$
[\mathcal{A}(\vec{u}+\vec{z})-\mathcal{A}(\vec{v}+\vec{z}), \vec{u}-\vec{v}] \geq \alpha\|\vec{u}-\vec{v}\|_{X}^{2},
$$

for all $\vec{z} \in X$, and for all $\vec{u}, \vec{v} \in V$, and

(iii) there exists $\beta>0$ such that

$$
\sup _{\substack{\vec{v} \in X \\ \vec{v} \neq 0}} \frac{[\mathcal{B}(\vec{v}), \tau]}{\|\vec{v}\|_{\mathrm{X}}} \geq \beta\|\tau\|_{Y} \quad \forall \tau \in Y .
$$

Then, for each $(\mathcal{F}, \mathcal{G}) \in X^{\prime} \times Y^{\prime}$ there exists a unique $(\vec{u}, \sigma) \in X \times Y$ such that

$$
\begin{aligned}
{[\mathcal{A}(\vec{u}), \vec{v}]+[\mathcal{B}(\vec{v}), \sigma] } & =[\mathcal{F}, \vec{v}] & & \forall \vec{v} \in X, \\
{[\mathcal{B}(\vec{u}), \tau] } & =[\mathcal{G}, \tau] & & \forall \tau \in Y .
\end{aligned}
$$

Moreover, there exist positive constants $C_{1}$ and $C_{2}$, depending only on $L, \alpha$, and $\beta$, such that

$$
\|\vec{u}\|_{X} \leq C_{1} \mathcal{M}(\mathcal{F}, \mathcal{G})
$$

and

$$
\|\sigma\|_{Y} \leq C_{2}\left\{\mathcal{M}(\mathcal{F}, \mathcal{G})+\sum_{j=1}^{2} \mathcal{M}(\mathcal{F}, \mathcal{G})^{p_{j}-1}\right\}
$$

where

$$
\mathcal{M}(\mathcal{F}, \mathcal{G}):=\|\mathcal{F}\|_{X^{\prime}}+\|\mathcal{G}\|_{Y^{\prime}}+\sum_{j=1}^{2}\|\mathcal{G}\|_{Y^{\prime}}^{p_{j}-1}+\|\mathcal{A}(0)\|_{X^{\prime}}
$$

Proof. We begin by noting that the unique solvability of problem (3.1) follows from hypotheses (i)-(iii) and a direct application of a slight modification of Theorem 3.1 in [13]. In fact, it suffices to observe that this latter result remains valid if the continuity and strict monotonicity hypotheses given by (ii) and (iii) of [13] are assumed to hold with different pairs $\left(p_{1}, p_{2}\right)$. Now, in order to obtain (3.2) and (3.3), and similarly to Theorem 3.1 of [13], we first note that $\vec{u}$ can be decomposed as

$$
\vec{u}=\vec{u}_{V}+\vec{u}_{\mathcal{G}}
$$

with $\vec{u}_{V} \in V$ and $\vec{u}_{\mathcal{G}} \in X$ satisfying

$$
\mathcal{B}\left(\vec{u}_{\mathcal{G}}\right)=\mathcal{G} \quad \text { and } \quad\left\|\vec{u}_{\mathcal{G}}\right\|_{X} \leq \frac{1}{\beta}\|\mathcal{G}\|_{Y^{\prime}}
$$

We notice that (3.6) is consequence of hypothesis (iii) and the open mapping theorem (cf. [21], Lems. A.36 and A.42). In turn, taking $\vec{v}=\vec{u}_{V} \in V$ in the first equation of (3.1), we have

$$
\left[\mathcal{A}\left(\vec{u}_{V}+\vec{u}_{\mathcal{G}}\right)-\mathcal{A}\left(0+\vec{u}_{\mathcal{G}}\right), \vec{u}_{V}\right]=\left[\mathcal{F}, \vec{u}_{V}\right]-\left[\mathcal{A}\left(\vec{u}_{\mathcal{G}}\right), \vec{u}_{V}\right]
$$


Then, combining hypothesis (i), (ii) and (3.6), we deduce that

$$
\alpha\left\|\vec{u}_{V}\right\|_{X}^{2} \leq\left\{\|\mathcal{F}\|_{X^{\prime}}+\left\|\mathcal{A}\left(\vec{u}_{\mathcal{G}}\right)\right\|_{X^{\prime}}\right\}\left\|\vec{u}_{V}\right\|_{X} \leq c_{1}\left\{\|\mathcal{F}\|_{X^{\prime}}+\|\mathcal{G}\|_{Y^{\prime}}+\sum_{j=1}^{2}\|\mathcal{G}\|_{Y^{\prime}}^{p_{j}-1}+\|\mathcal{A}(0)\|_{X^{\prime}}\right\}\left\|\vec{u}_{V}\right\|_{X},
$$

with $c_{1}>0$ depending only on $\beta$ and $L$, which yields

$$
\left\|\vec{u}_{V}\right\|_{X} \leq \frac{c_{1}}{\alpha}\left\{\|\mathcal{F}\|_{X^{\prime}}+\|\mathcal{G}\|_{Y^{\prime}}+\sum_{j=1}^{2}\|\mathcal{G}\|_{Y^{\prime}}^{p_{j}-1}+\|\mathcal{A}(0)\|_{X^{\prime}}\right\} .
$$

In this way, employing (3.6) and (3.7) in (3.5), we deduce (3.2). On the other hand, from the first equation of (3.1), and combining hypotheses (iii) and (i), we find that

$$
\|\sigma\|_{Y} \leq \frac{1}{\beta}\left\{\|\mathcal{F}\|_{X^{\prime}}+\|\mathcal{A}(\vec{u})\|_{X^{\prime}}\right\} \leq c_{2}\left\{\|\mathcal{F}\|_{X^{\prime}}+\|\vec{u}\|_{X}+\sum_{j=1}^{2}\|\vec{u}\|_{X}^{p_{j}-1}+\|\mathcal{A}(0)\|_{X^{\prime}}\right\},
$$

with $c_{2}>0$ depending only on $\beta$ and $L$. Then, equations (3.2) and (3.8) implies (3.3), which ends the proof.

Next, we establish the stability properties of the operators and functionals involved in (2.27). We begin by observing that the linear operators $b, \widetilde{a}_{j}$, and $\widetilde{b}, j \in\{1,2\}$, satisfy the boundedness estimates

$$
\begin{aligned}
|[b(\overrightarrow{\mathbf{v}}), \boldsymbol{\tau}]| & \leq\|\overrightarrow{\mathbf{v}}\|\|\boldsymbol{\tau}\|_{\operatorname{div}_{3 / 2} ; \Omega} & & \forall \overrightarrow{\mathbf{v}} \in \mathbf{H}, \forall \boldsymbol{\tau} \in \mathbb{H}_{0}\left(\operatorname{div}_{3 / 2} ; \Omega\right), \\
\left|\left[\widetilde{a}_{j}\left(\vec{\phi}_{j}\right), \vec{\psi}_{j}\right]\right| & \leq\left\|\mathbf{Q}_{j}\right\|_{0, \infty, \Omega}\left\|\vec{\phi}_{j}\right\|\left\|\vec{\psi}_{j}\right\| & & \forall \vec{\phi}_{j}, \vec{\psi}_{j} \in \widetilde{\mathbf{H}}, \\
\left|\left[\widetilde{b}\left(\vec{\psi}_{j}\right), \boldsymbol{\eta}_{j}\right]\right| & \leq\left\|\vec{\psi}_{j}\right\|\left\|\boldsymbol{\eta}_{j}\right\|_{\operatorname{div}_{6 / 5} ; \Omega} & & \forall \vec{\psi}_{j} \in \widetilde{\mathbf{H}}, \forall \boldsymbol{\eta}_{j} \in \mathbf{H}\left(\operatorname{div}_{6 / 5} ; \Omega\right),
\end{aligned}
$$

where

$$
\mathbf{H}:=\mathbf{L}^{3}(\Omega) \times \mathbb{L}_{\mathrm{tr}}^{2}(\Omega) \quad \text { and } \quad \widetilde{\mathbf{H}}:=\mathrm{L}^{6}(\Omega) \times \mathbf{L}^{2}(\Omega) .
$$

In turn, employing the Cauchy-Schwarz and Hölder inequalities, and recalling the definition of $\mathbf{f}(c f .(2.2))$, it is readily seen that, given $\varphi \in \mathbf{L}^{6}(\Omega)$, the functionals $G_{\mathrm{D}}, F_{\boldsymbol{\varphi}}$ and $\widetilde{G}_{j}(c f .(2.17),(2.18)$ and (2.26)) satisfy

$$
\begin{aligned}
\left|\left[G_{D}, \boldsymbol{\tau}\right]\right| & \leq C_{D}\left\|\mathbf{u}_{\mathrm{D}}\right\|_{1 / 2, \Gamma}\|\boldsymbol{\tau}\|_{\operatorname{div}_{3 / 2} ; \Omega} & & \forall \boldsymbol{\tau} \in \mathbb{H}\left(\operatorname{div}_{3 / 2} ; \Omega\right), \\
\left|\left[F_{\boldsymbol{\varphi}}, \overrightarrow{\mathbf{v}}\right]\right| & \leq\|\mathbf{g}\|_{0, \Omega}\left(\|\boldsymbol{\varphi}\|_{0,6 ; \Omega}+\left\|\phi_{\mathrm{r}}\right\|_{0,6 ; \Omega}\right)\|\overrightarrow{\mathbf{v}}\| & & \forall \overrightarrow{\mathbf{v}} \in \mathbf{H}, \\
\left|\left[\widetilde{G}_{j}, \boldsymbol{\eta}_{j}\right]\right| & \leq \widetilde{C}_{D}\left\|\phi_{j, \mathrm{D}}\right\|_{1 / 2, \Gamma}\left\|\boldsymbol{\eta}_{j}\right\|_{\operatorname{div}_{6 / 5} ; \Omega} & & \forall \boldsymbol{\eta}_{j} \in \mathbf{H}\left(\operatorname{div}_{6 / 5} ; \Omega\right),
\end{aligned}
$$

where $\phi_{\mathrm{r}}:=\left(\phi_{1, \mathrm{r}}, \phi_{2, \mathrm{r}}\right) \in \mathbf{L}^{6}(\Omega)$, and $C_{D}$ and $\widetilde{C}_{D}$ are positive constants depending on $\left\|i_{p}\right\|$, the norm of the injection of $\mathrm{H}^{1}(\Omega)$ into $\mathrm{L}^{p}(\Omega)$, with $p$ equal to 3 and 6 , respectively (see [10], Eq. (4.4) and [11], Lem. 3.5 for details).

We end this section by collecting the inf-sup conditions for the operators $b$ and $\widetilde{b}(c f .(2.16)$ and (2.25)), and by stating some fundamental properties of the operator $c_{j}(\mathbf{w})(c f .(2.24))$, whose proofs follow from a slight adaptation of Lemmas 3.3 and 3.4 in [18], respectively, reason why details are omitted.

Lemma 3.2. There exist positive constants $\beta$ and $\widetilde{\beta}$, such that

$$
\sup _{\substack{\overrightarrow{\mathbf{v}} \in \mathbf{H} \\ \overrightarrow{\mathbf{v}} \neq \mathbf{0}}} \frac{[b(\overrightarrow{\mathbf{v}}), \boldsymbol{\tau}]}{\|\overrightarrow{\mathbf{v}}\|} \geq \beta\|\boldsymbol{\tau}\|_{\operatorname{div}_{3 / 2} ; \Omega} \quad \forall \boldsymbol{\tau} \in \mathbb{H}_{0}\left(\operatorname{div}_{3 / 2} ; \Omega\right)
$$


and

$$
\sup _{\substack{\vec{\psi} \in \widetilde{\mathbf{H}} \\ \vec{\psi} \neq 0}} \frac{[\widetilde{b}(\vec{\psi}), \boldsymbol{\eta}]}{\|\vec{\psi}\|} \geq \widetilde{\beta}\|\boldsymbol{\eta}\|_{\operatorname{div}_{6 / 5} ; \Omega} \quad \forall \boldsymbol{\eta} \in \mathbf{H}\left(\operatorname{div}_{6 / 5} ; \Omega\right)
$$

Lemma 3.3. The operator $c_{j}(\mathbf{w}): \widetilde{\mathbf{H}} \rightarrow \widetilde{\mathbf{H}}^{\prime}, j \in\{1,2\}$, is bounded for each $\mathbf{w} \in \mathbf{L}^{3}(\Omega)$ with boundedness constant given by $\mathrm{R}_{j}\|\mathbf{w}\|_{0,3 ; \Omega}$, and there hold the following additional properties

$$
\begin{aligned}
{\left[c_{j}(\mathbf{w})\left(\vec{\psi}_{j}\right), \vec{\psi}_{j}\right] } & =0 & & \forall \vec{\psi}_{j} \in \widetilde{\mathbf{H}}, \\
\left|\left[c_{j}(\mathbf{w})\left(\vec{\phi}_{j}\right)-c_{j}(\mathbf{z})\left(\vec{\phi}_{j}\right), \vec{\psi}_{j}\right]\right| & \leq \mathbf{R}_{j}\|\mathbf{w}-\mathbf{z}\|_{0,3 ; \Omega}\left\|\vec{\phi}_{j}\right\|\left\|\vec{\psi}_{j}\right\| & & \forall \mathbf{w}, \mathbf{z} \in \mathbf{L}^{3}(\Omega), \forall \vec{\phi}_{j}, \vec{\psi}_{j} \in \widetilde{\mathbf{H}} .
\end{aligned}
$$

\subsection{A fixed point strategy}

In what follows we proceed similarly to $[17]$ (see also $[14,18,19]$ ) and utilize a fixed point strategy to prove the well-posedness of (2.27). Let us first define the operator $\mathbf{S}: \mathbf{L}^{6}(\Omega) \rightarrow \mathbf{L}^{3}(\Omega)$ as

$$
\mathbf{S}(\varphi):=\mathbf{u} \quad \forall \varphi \in \mathbf{L}^{6}(\Omega),
$$

where $(\overrightarrow{\mathbf{u}}, \boldsymbol{\sigma}):=((\mathbf{u}, \mathbf{t}), \boldsymbol{\sigma}) \in \mathbf{H} \times \mathbb{H}_{0}\left(\operatorname{div}_{3 / 2} ; \Omega\right)$ is the unique solution (to be confirmed below) of the problem

$$
\begin{aligned}
{[a(\overrightarrow{\mathbf{u}}), \overrightarrow{\mathbf{v}}]+[b(\overrightarrow{\mathbf{v}}), \boldsymbol{\sigma}] } & =\left[F_{\boldsymbol{\varphi}}, \overrightarrow{\mathbf{v}}\right] & & \forall \overrightarrow{\mathbf{v}} \in \mathbf{H}, \\
{[b(\overrightarrow{\mathbf{u}}), \boldsymbol{\tau}] } & =\left[G_{\mathrm{D}}, \boldsymbol{\tau}\right] & & \forall \boldsymbol{\tau} \in \mathbb{H}_{0}\left(\operatorname{div}_{3 / 2} ; \Omega\right) .
\end{aligned}
$$

In turn, for each $j \in\{1,2\}$ we let $\widetilde{\mathbf{S}}_{j}: \mathbf{L}^{3}(\Omega) \rightarrow \mathrm{L}^{6}(\Omega)$ be the operator given by

$$
\widetilde{\mathbf{S}}_{j}(\mathbf{w}):=\phi_{j} \quad \forall \mathbf{w} \in \mathbf{L}^{3}(\Omega),
$$

where $\left(\vec{\phi}_{j}, \boldsymbol{\rho}_{j}\right):=\left(\left(\phi_{j}, \widetilde{\mathbf{t}}_{j}\right), \boldsymbol{\rho}_{j}\right) \in \widetilde{\mathbf{H}} \times \mathbf{H}\left(\operatorname{div}_{6 / 5} ; \Omega\right)$ is the unique solution (to be confirmed below) of the problem

$$
\begin{aligned}
{\left[\widetilde{a}_{j}\left(\vec{\phi}_{j}\right), \vec{\psi}_{j}\right]+\left[c_{j}(\mathbf{w})\left(\vec{\phi}_{j}\right), \vec{\psi}_{j}\right]+\left[\widetilde{b}\left(\vec{\psi}_{j}\right), \boldsymbol{\rho}_{j}\right] } & =0 & & \forall \vec{\psi}_{j} \in \widetilde{\mathbf{H}}, \\
{\left[\widetilde{b}\left(\vec{\phi}_{j}\right), \boldsymbol{\eta}_{j}\right] } & =\left[\widetilde{G}_{j}, \boldsymbol{\eta}_{j}\right] & & \forall \boldsymbol{\eta}_{j} \in \mathbf{H}\left(\operatorname{div}_{6 / 5} ; \Omega\right) .
\end{aligned}
$$

Then, we can introduce $\widetilde{\mathbf{S}}(\mathbf{w}):=\left(\widetilde{\mathbf{S}}_{1}(\mathbf{w}), \widetilde{\mathbf{S}}_{2}(\mathbf{w})\right) \in \mathbf{L}^{6}(\Omega)$ for all $\mathbf{w} \in \mathbf{L}^{3}(\Omega)$. Consequently, we set the operator $\mathbf{T}: \mathbf{L}^{3}(\Omega) \rightarrow \mathbf{L}^{3}(\Omega)$ as

$$
\mathbf{T}(\mathbf{w}):=\mathbf{S}(\widetilde{\mathbf{S}}(\mathbf{w})) \quad \forall \mathbf{w} \in \mathbf{L}^{3}(\Omega),
$$

and realize that solving (2.27) is equivalent to finding $\mathbf{u} \in \mathbf{L}^{3}(\Omega)$ such that

$$
\mathbf{T}(\mathbf{u})=\mathbf{u} .
$$

\subsection{Well-definedness of the fixed point operator}

In this section we show that the uncoupled problems (3.20) and (3.22) are well-posed, which means, equivalently, that $\mathbf{S}$ and $\widetilde{\mathbf{S}}$ ( $c f$. (3.19) and (3.21)) are indeed well-defined. We begin with the operator $\mathbf{S}$. To this end, we first observe that, given $\varphi \in \mathbf{L}^{6}(\Omega)$, the problem (3.20) has the same structure as the one in Theorem 3.1. Therefore, in order to apply this abstract result, we notice that, thanks to the uniform convexity and separability of $L^{p}(\Omega)$ for $p \in(1,+\infty)$, all the spaces involved in (3.20), that is, $\mathbf{L}^{3}(\Omega), \mathbb{L}_{\mathrm{tr}}^{2}(\Omega)$ and $\mathbb{H}_{0}\left(\operatorname{div}_{3 / 2} ; \Omega\right)$, share the same properties.

We continue our analysis by proving that the nonlinear operator $a(c f .(2.15))$ satisfies hypothesis (i) of Theorem 3.1 with $p_{1}=3$ and $p_{2}=2$. 
Lemma 3.4. Let us define $L_{\mathrm{BF}}:=\max \left\{|\Omega|^{1 / 3}\left\|\mathbf{K}^{-1}\right\|_{0, \infty, \Omega}, \mathrm{F}, \nu\right\}$. Then, there holds

$$
\|a(\overrightarrow{\mathbf{u}})-a(\overrightarrow{\mathbf{v}})\|_{\mathbf{H}^{\prime}} \leq L_{\mathrm{BF}}\left\{\|\mathbf{u}-\mathbf{v}\|_{0,3 ; \Omega}+\|\mathbf{t}-\mathbf{r}\|_{0, \Omega}+\left(\|\mathbf{u}\|_{0,3 ; \Omega}+\|\mathbf{v}\|_{0,3 ; \Omega}\right)\|\mathbf{u}-\mathbf{v}\|_{0,3 ; \Omega}\right\},
$$

for all $\overrightarrow{\mathbf{u}}=(\mathbf{u}, \mathbf{t}), \overrightarrow{\mathbf{v}}=(\mathbf{v}, \mathbf{r}) \in \mathbf{H}$.

Proof. It follows straightforwardly from the definition of $a$ ( $c f .(2.15))$, along with the triangle, Cauchy-Schwarz, and Hölder's inequalities. Further details are omitted.

Now, let us look at the kernel of the operator $b(c f .(2.16))$, that is

$$
\mathbf{V}:=\left\{\overrightarrow{\mathbf{v}}=(\mathbf{v}, \mathbf{r}) \in \mathbf{H}:[b(\overrightarrow{\mathbf{v}}), \boldsymbol{\tau}]=0 \quad \forall \boldsymbol{\tau} \in \mathbb{H}_{0}\left(\operatorname{div}_{3 / 2} ; \Omega\right)\right\},
$$

which, proceeding similarly to equation (3.34) of [18], reduces to

$$
\mathbf{V}:=\left\{\overrightarrow{\mathbf{v}}=(\mathbf{v}, \mathbf{r}) \in \mathbf{H}: \nabla \mathbf{v}=\mathbf{r} \quad \text { and } \quad \mathbf{v} \in \mathbf{H}_{0}^{1}(\Omega)\right\} .
$$

The following lemma establishes hypothesis (ii) of Theorem 3.1 for $a$.

Lemma 3.5. The family of operators $\left\{a(\cdot+\overrightarrow{\mathbf{z}}): \mathbf{V} \rightarrow \mathbf{V}^{\prime}: \overrightarrow{\mathbf{z}} \in \mathbf{H}\right\}$ is uniformly strongly monotone, that is, there exists $\alpha_{\mathrm{BF}}>0$, such that

$$
[a(\overrightarrow{\mathbf{u}}+\overrightarrow{\mathbf{z}})-a(\overrightarrow{\mathbf{v}}+\overrightarrow{\mathbf{z}}), \overrightarrow{\mathbf{u}}-\overrightarrow{\mathbf{v}}] \geq \alpha_{\mathrm{BF}}\|\overrightarrow{\mathbf{u}}-\overrightarrow{\mathbf{v}}\|^{2},
$$

for all $\overrightarrow{\mathbf{z}}=(\mathbf{z}, \mathbf{s}) \in \mathbf{H}$, and for all $\overrightarrow{\mathbf{u}}=(\mathbf{u}, \mathbf{t}), \overrightarrow{\mathbf{v}}=(\mathbf{v}, \mathbf{r}) \in \mathbf{V}$.

Proof. Let $\overrightarrow{\mathbf{z}}=(\mathbf{z}, \mathbf{s}) \in \mathbf{H}$ and $\overrightarrow{\mathbf{u}}=(\mathbf{u}, \mathbf{t}), \overrightarrow{\mathbf{v}}=(\mathbf{v}, \mathbf{r}) \in \mathbf{V}$. Bearing in mind the definition of $a(c f$. (2.15)), and using (2.3), we obtain

$$
[a(\overrightarrow{\mathbf{u}}+\overrightarrow{\mathbf{z}})-a(\overrightarrow{\mathbf{v}}+\overrightarrow{\mathbf{z}}), \overrightarrow{\mathbf{u}}-\overrightarrow{\mathbf{v}}] \geq C_{\mathbf{K}}\|\mathbf{u}-\mathbf{v}\|_{0, \Omega}^{2}+\mathrm{F} \int_{\Omega}(|\mathbf{u}+\mathbf{z}|(\mathbf{u}+\mathbf{z})-|\mathbf{v}+\mathbf{z}|(\mathbf{v}+\mathbf{z})) \cdot(\mathbf{u}-\mathbf{v})+\nu\|\mathbf{t}-\mathbf{r}\|_{0, \Omega}^{2} .
$$

Hence, thanks to Lemma 2.1, equation (2.1b) of [3] with $p=3$, there exists $c_{1}(\Omega)>0$, depending only on $|\Omega|$, such that

$$
\int_{\Omega}(|\mathbf{u}+\mathbf{z}|(\mathbf{u}+\mathbf{z})-|\mathbf{v}+\mathbf{z}|(\mathbf{v}+\mathbf{z})) \cdot(\mathbf{u}-\mathbf{v}) \geq c_{1}(\Omega)\|\mathbf{u}-\mathbf{v}\|_{0,3 ; \Omega}^{3},
$$

which, together with (3.28), yields

$$
[a(\overrightarrow{\mathbf{u}}+\overrightarrow{\mathbf{z}})-a(\overrightarrow{\mathbf{v}}+\overrightarrow{\mathbf{z}}), \overrightarrow{\mathbf{u}}-\overrightarrow{\mathbf{v}}] \geq C_{\mathbf{K}}\|\mathbf{u}-\mathbf{v}\|_{0, \Omega}^{2}+c_{1}(\Omega) \mathbf{F}\|\mathbf{u}-\mathbf{v}\|_{0,3 ; \Omega}^{3}+\nu\|\mathbf{t}-\mathbf{r}\|_{0, \Omega}^{2}
$$

Next, bounding below the second term on the right hand side of (3.29) by 0 , employing the fact that $\mathbf{t}-\mathbf{r}=$ $\nabla(\mathbf{u}-\mathbf{v})$ in $\Omega$ and $\mathbf{u}-\mathbf{v} \in \mathbf{H}_{0}^{1}(\Omega)(c f .(3.26))$, and using the continuous injection $\mathbf{i}_{3}$ of $\mathbf{H}^{1}(\Omega)$ into $\mathbf{L}^{3}(\Omega)$ (see, e.g. [30], Thm. 1.3.4), we deduce that

$$
\begin{aligned}
{[a(\overrightarrow{\mathbf{u}}+\overrightarrow{\mathbf{z}})-a(\overrightarrow{\mathbf{v}}+\overrightarrow{\mathbf{z}}), \overrightarrow{\mathbf{u}}-\overrightarrow{\mathbf{v}}] } & \geq \min \left\{C_{\mathbf{K}}, \frac{\nu}{2}\right\}\left\{\|\mathbf{u}-\mathbf{v}\|_{1, \Omega}^{2}+\|\mathbf{t}-\mathbf{r}\|_{0, \Omega}^{2}\right\} \\
& \geq \min \left\{C_{\mathbf{K}}, \frac{\nu}{2}\right\}\left\{\left\|\mathbf{i}_{3}\right\|^{-2}\|\mathbf{u}-\mathbf{v}\|_{0,3 ; \Omega}^{2}+\|\mathbf{t}-\mathbf{r}\|_{0, \Omega}^{2}\right\}
\end{aligned}
$$

which yields (3.27) with $\alpha_{\mathrm{BF}}:=\min \left\{C_{\mathbf{K}}, \frac{\nu}{2}\right\} \min \left\{1,\left\|\mathbf{i}_{3}\right\|^{-2}\right\}$.

As a corollary of Lemma 3.5, taking in particular $\overrightarrow{\mathbf{u}}-\overrightarrow{\mathbf{v}}, \mathbf{0} \in \mathbf{V}$ and $\overrightarrow{\mathbf{z}}=\overrightarrow{\mathbf{v}} \in \mathbf{H}$ in (3.27), we arrive at

$$
[a(\overrightarrow{\mathbf{u}})-a(\overrightarrow{\mathbf{v}}), \overrightarrow{\mathbf{u}}-\overrightarrow{\mathbf{v}}] \geq \alpha_{\mathrm{BF}}\|\overrightarrow{\mathbf{u}}-\overrightarrow{\mathbf{v}}\|^{2},
$$

for all $\overrightarrow{\mathbf{u}}, \overrightarrow{\mathbf{v}} \in \mathbf{H}$ such that $\overrightarrow{\mathbf{u}}-\overrightarrow{\mathbf{v}} \in \mathbf{V}$.

We now establish the unique solvability of the nonlinear problem (3.20). 
Lemma 3.6. For each $\boldsymbol{\varphi} \in \mathbf{L}^{6}(\Omega)$, the problem (3.20) has a unique solution $(\overrightarrow{\mathbf{u}}, \boldsymbol{\sigma}):=((\mathbf{u}, \mathbf{t}), \boldsymbol{\sigma}) \in \mathbf{H} \times$ $\mathbb{H}_{0}\left(\operatorname{div}_{3 / 2} ; \Omega\right)$. Moreover, there exists a positive constant $C_{\mathbf{S}}$, independent of $\boldsymbol{\varphi}$, such that

$$
\|\mathbf{S}(\boldsymbol{\varphi})\|_{0,3 ; \Omega} \leq\|\overrightarrow{\mathbf{u}}\| \leq C_{\mathbf{S}}\left\{\|\mathbf{g}\|_{0, \Omega}\left(\|\boldsymbol{\varphi}\|_{0,6 ; \Omega}+\left\|\phi_{\mathbf{r}}\right\|_{0,6 ; \Omega}\right)+\left\|\mathbf{u}_{\mathrm{D}}\right\|_{1 / 2, \Gamma}+\left\|\mathbf{u}_{\mathrm{D}}\right\|_{1 / 2, \Gamma}^{2}\right\} .
$$

Proof. Given $\varphi \in \mathbf{L}^{6}(\Omega)$, we first recall from (3.9), (3.12) and (3.13) that $b, G_{\mathrm{D}}$ and $F_{\boldsymbol{\varphi}}$ are all linear and bounded. Thus, bearing in mind Lemmas 3.4 and 3.5, and the inf-sup condition of $b$ given by (3.15) (cf. Lem. 3.2), a straightforward application of Theorem 3.1, with $p_{1}=3$ and $p_{2}=2$, to problem (3.20) completes the proof. In particular, noting from (2.15) that $a(\mathbf{0})$ is the null functional, we get from (3.4) that

$$
\mathcal{M}\left(F_{\boldsymbol{\varphi}}, G_{\mathrm{D}}\right)=\left\|F_{\boldsymbol{\varphi}}\right\|+2\left\|G_{\mathrm{D}}\right\|+\left\|G_{\mathrm{D}}\right\|^{2},
$$

and hence the a priori estimate (3.2) yields

$$
\|\overrightarrow{\mathbf{u}}\| \leq C_{1}\left\{\left\|F_{\varphi}\right\|+\left\|G_{\mathrm{D}}\right\|+\left\|G_{\mathrm{D}}\right\|^{2}\right\},
$$

with a positive constant $C_{1}$ depending only on $L_{\mathrm{BF}}, \alpha_{\mathrm{BF}}$, and $\beta$. The foregoing inequality together with the bounds of $\left\|G_{\mathrm{D}}\right\|$ and $\left\|F_{\boldsymbol{\varphi}}\right\|$ (cf. (3.12) and (3.13)) imply (3.31) with $C_{\mathbf{S}}$ depending only on $\left\|\mathbf{i}_{3}\right\|, L_{\mathrm{BF}}, \alpha_{\mathrm{BF}}$, and $\beta$, thus completing the proof.

For later use in the paper we note here that, applying (3.3), and using again the bounds (3.12) and (3.13) for $\left\|G_{\mathrm{D}}\right\|$ and $\left\|F_{\varphi}\right\|$, respectively, the a priori estimate for the second component of the solution to the problem defining $\mathbf{S}(c f .(3.20))$ reduces to

$$
\|\boldsymbol{\sigma}\|_{\operatorname{div}_{3 / 2} ; \Omega} \leq C_{\boldsymbol{\sigma}} \sum_{j=1}^{2}\left\{\left(\|\mathbf{g}\|_{0, \Omega}\left(\|\boldsymbol{\varphi}\|_{0,6 ; \Omega}+\left\|\phi_{\mathrm{r}}\right\|_{0,6 ; \Omega}\right)+\left\|\mathbf{u}_{\mathrm{D}}\right\|_{1 / 2, \Gamma}+\left\|\mathbf{u}_{\mathrm{D}}\right\|_{1 / 2, \Gamma}^{2}\right)^{j}\right\},
$$

with $C_{\boldsymbol{\sigma}}$ depending only on $\left\|\mathbf{i}_{3}\right\|, L_{\mathrm{BF}}, \alpha_{\mathrm{BF}}$, and $\beta$.

Next, we aim to proving the well-posedness of problem (3.22), or, equivalently, the well-definedness of the operator $\widetilde{\mathbf{S}}(c f .(3.21))$, for which, following Lemma 3.6 of [18], we first establish the corresponding hypotheses required by the Babuška-Brezzi theory in Banach spaces. In this way, and similarly to (3.26) and equation (3.35) of [18], we first let $\widetilde{\mathbf{V}}$ be the kernel of the operator $\widetilde{b}(c f .(2.25))$, that is

$$
\widetilde{\mathbf{V}}:=\left\{\vec{\psi}=(\psi, \widetilde{\mathbf{r}}) \in \widetilde{\mathbf{H}}: \nabla \psi=\widetilde{\mathbf{r}} \text { and } \psi \in \mathrm{H}_{0}^{1}(\Omega)\right\} .
$$

Then the $\widetilde{\mathbf{V}}$-ellipticity of the operator $\widetilde{a}_{j}$ is stated as follows.

Lemma 3.7. There exists a positive constant $\widetilde{\alpha}_{j}$ such that

$$
\left[\widetilde{a}_{j}\left(\vec{\psi}_{j}\right), \vec{\psi}_{j}\right] \geq \widetilde{\alpha}_{j}\left\|\vec{\psi}_{j}\right\|^{2} \quad \forall \vec{\psi}_{j}:=\left(\psi_{j}, \widetilde{\mathbf{r}}_{j}\right) \in \widetilde{\mathbf{V}} .
$$

Proof. We proceed as in Lemma 3.2 of [18]. In fact, given $\vec{\psi}_{j}:=\left(\psi_{j}, \widetilde{\mathbf{r}}_{j}\right) \in \widetilde{\mathbf{V}}$, we know from (3.33) that $\nabla \psi_{j}=\widetilde{\mathbf{r}}_{j}$ and $\psi_{j} \in \mathrm{H}_{0}^{1}(\Omega)$. Hence, using the fact that $\mathbf{Q}_{j}$ is a uniformly positive definite tensor $(c f$. (2.3)), and resorting to the Poincaré inequality with positive constant $c_{P}$, and to the continuous injection $i_{6}$ of $\mathrm{H}^{1}(\Omega)$ into $\mathrm{L}^{6}(\Omega)$ (see, e.g. [30], Thm. 1.3.4), we obtain

$$
\left[\widetilde{a}_{j}\left(\vec{\psi}_{j}\right), \vec{\psi}_{j}\right] \geq C_{\mathbf{Q}_{j}}\left\|\widetilde{\mathbf{r}}_{j}\right\|_{0, \Omega}^{2}=\frac{C_{\mathbf{Q}_{j}}}{2}\left\{\left\|\widetilde{\mathbf{r}}_{j}\right\|_{0, \Omega}^{2}+\left\|\nabla \psi_{j}\right\|_{0, \Omega}^{2}\right\} \geq \frac{C_{\mathbf{Q}_{j}}}{2}\left\{\left\|\widetilde{\mathbf{r}}_{j}\right\|_{0, \Omega}^{2}+c_{P}^{-1}\left\|i_{6}\right\|^{-2}\left\|\psi_{j}\right\|_{0,6 ; \Omega}^{2}\right\},
$$

which gives (3.34) with $\widetilde{\alpha}_{j}:=\frac{C_{\mathbf{Q}_{j}}}{2} \min \left\{1, c_{P}^{-1}\left\|i_{6}\right\|^{-2}\right\}$. 
We are now in position to provide the announced result. More precisely, denoting

$$
\|\mathbf{Q}\|_{0, \infty ; \Omega}:=\left\|\mathbf{Q}_{1}\right\|_{0, \infty ; \Omega}+\left\|\mathbf{Q}_{2}\right\|_{0, \infty ; \Omega} \quad \text { and } \quad\left\|\phi_{\mathrm{D}}\right\|_{1 / 2, \Gamma}:=\left\|\phi_{1, \mathrm{D}}\right\|_{1 / 2, \Gamma}+\left\|\phi_{2, \mathrm{D}}\right\|_{1 / 2, \Gamma}
$$

we have the following lemma.

Lemma 3.8. For each $\mathbf{w} \in \mathbf{L}^{3}(\Omega)$, and $j \in\{1,2\}$, problem $(3.22)$ has a unique solution $\left(\vec{\phi}_{j}, \boldsymbol{\rho}_{j}\right):=$ $\left(\left(\phi_{j}, \widetilde{\mathbf{t}}_{j}\right), \boldsymbol{\rho}_{j}\right) \in \widetilde{\mathbf{H}} \times \mathbf{H}\left(\operatorname{div}_{6 / 5} ; \Omega\right)$. Moreover, there exists a positive constant $C_{\widetilde{\mathbf{S}}}$, independent of $\mathbf{w}$, such that

$$
\|\widetilde{\mathbf{S}}(\mathbf{w})\|_{0,6 ; \Omega} \leq \sum_{j=1}^{2}\left\|\vec{\phi}_{j}\right\| \leq C_{\widetilde{\mathbf{S}}}\left(1+\|\mathbf{Q}\|_{0, \infty ; \Omega}+\|\mathbf{w}\|_{0,3 ; \Omega}\right)\left\|\phi_{\mathrm{D}}\right\|_{1 / 2, \Gamma}
$$

Proof. We proceed as in Lemma 3.5 of [18]. In fact, given $\mathbf{w} \in \mathbf{L}^{3}(\Omega)$ and $j \in\{1,2\}$, we introduce the operator $\mathcal{A}_{j}(\mathbf{w}): \widetilde{\mathbf{H}} \rightarrow \widetilde{\mathbf{H}}^{\prime}$ defined by

$$
\left[\mathcal{A}_{j}(\mathbf{w})\left(\vec{\phi}_{j}\right), \vec{\psi}_{j}\right]:=\left[\widetilde{a}_{j}\left(\vec{\phi}_{j}\right), \vec{\psi}_{j}\right]+\left[c_{j}(\mathbf{w})\left(\vec{\phi}_{j}\right), \vec{\psi}_{j}\right] \quad \forall \vec{\phi}_{j}, \vec{\psi}_{j} \in \widetilde{\mathbf{H}},
$$

where $\widetilde{a}_{j}$ and $c_{j}(\mathbf{w})$ are the operators defined in (2.23) and (2.24), respectively. Then, the problem (3.22) can be reformulated as: Find $\left(\vec{\phi}_{j}, \boldsymbol{\rho}_{j}\right) \in \widetilde{\mathbf{H}} \times \mathbf{H}\left(\operatorname{div}_{6 / 5} ; \Omega\right)$ such that

$$
\begin{aligned}
{\left[\mathcal{A}_{j}(\mathbf{w})\left(\vec{\phi}_{j}\right), \vec{\psi}_{j}\right]+\left[\widetilde{b}\left(\vec{\psi}_{j}\right), \boldsymbol{\rho}_{j}\right] } & =0 & & \forall \vec{\psi}_{j} \in \widetilde{\mathbf{H}}, \\
{\left[\widetilde{b}\left(\vec{\phi}_{j}\right), \boldsymbol{\eta}_{j}\right] } & =\left[\widetilde{G}_{j}, \boldsymbol{\eta}_{j}\right] & & \forall \boldsymbol{\eta}_{j} \in \mathbf{H}\left(\operatorname{div}_{6 / 5} ; \Omega\right) .
\end{aligned}
$$

Next, we observe from (3.10) and Lemma 3.3 that $\mathcal{A}_{j}(\mathbf{w})$ is bounded, that is there holds

$$
\left|\left[\mathcal{A}_{j}(\mathbf{w})\left(\vec{\phi}_{j}\right), \vec{\psi}_{j}\right]\right| \leq\left(\left\|\mathbf{Q}_{j}\right\|_{0, \infty ; \Omega}+\mathrm{R}_{j}\|\mathbf{w}\|_{0,3 ; \Omega}\right)\left\|\vec{\phi}_{j}\right\|\left\|\vec{\psi}_{j}\right\| \quad \forall \vec{\phi}_{j}, \vec{\psi}_{j} \in \widetilde{\mathbf{H}}
$$

In addition, it is clear from (3.34) and (3.17) that $\mathcal{A}_{j}(\mathbf{w})$ is elliptic on $\widetilde{\mathbf{V}}(c f .(3.33))$ with the same constant $\widetilde{\alpha}_{j}$ from (3.34). In turn, recalling that the bounded linear operator $\widetilde{b}$ satisfies the inf-sup condition (3.16) (cf. Lem. 3.2) and that $\widetilde{G}_{j}$ is a bounded linear functional ( $c f .(3.14)$ ), a direct application of the Babuška-Brezzi theory in Banach spaces guarantees that (3.37) is well-posed. Moreover, the corresponding a priori estimate provided by that theory ( $c f$. [21], Eq. (2.30), Thm. 2.34), and the continuity bounds of $\widetilde{G}_{j}$ and $\mathcal{A}_{j}(\mathbf{w})(c f .(3.14)$, (3.38)), imply

$$
\left\|\vec{\phi}_{j}\right\| \leq \frac{\widetilde{C}_{\mathrm{D}}}{\widetilde{\beta}}\left(1+\frac{\left\|\mathbf{Q}_{j}\right\|_{0, \infty ; \Omega}+\mathrm{R}_{j}\|\mathbf{w}\|_{0,3 ; \Omega}}{\widetilde{\alpha}_{j}}\right)\left\|\phi_{j, \mathrm{D}}\right\|_{1 / 2, \Gamma},
$$

which yields (3.35) with $C_{\widetilde{\mathbf{S}}}:=\max \left\{C_{\widetilde{\mathbf{S}}_{1}}, C_{\widetilde{\mathbf{S}}_{2}}\right\}$ and $C_{\widetilde{\mathbf{S}}_{j}}:=\widetilde{\alpha}_{j}^{-1} \widetilde{\beta}^{-1} \widetilde{C}_{\mathrm{D}} \max \left\{1, \widetilde{\alpha}_{j}, \mathrm{R}_{j}\right\}$.

Similarly as for the derivation of (3.32), we notice that, applying the second a priori estimate from equation (2.30), Theorem 2.34 of [21], and employing (3.14) and (3.38) to bound $\left\|\widetilde{G}_{j}\right\|$ and $\left\|\mathcal{A}_{j}(\mathbf{w})\right\|$, respectively, the second component of the solution to the problem defining $\widetilde{\mathbf{S}}_{j}(c f .(3.22))$ can be bounded as

$$
\left\|\boldsymbol{\rho}_{j}\right\|_{\operatorname{div}_{6 / 5} ; \Omega} \leq \frac{\widetilde{C}_{\mathrm{D}}}{\widetilde{\beta}^{2}}\left(\left\|\mathbf{Q}_{j}\right\|_{0, \infty ; \Omega}+\mathrm{R}_{j}\|\mathbf{w}\|_{0,3 ; \Omega}\right)\left(1+\frac{\left\|\mathbf{Q}_{j}\right\|_{0, \infty ; \Omega}+\mathrm{R}_{j}\|\mathbf{w}\|_{0,3 ; \Omega}}{\widetilde{\alpha}_{j}}\right)\left\|\phi_{j, \mathrm{D}}\right\|_{1 / 2, \Gamma}
$$




\subsection{Solvability analysis of the fixed-point equation}

Having proved the well-posedness of the uncoupled problems (3.20) and (3.22), which ensures that the operators $\mathbf{S}, \widetilde{\mathbf{S}}$ and $\mathbf{T}$ are well defined, we now aim to establish the existence of a unique fixed-point of the operator T. For this purpose, in what follows we will verify the hypothesis of the Banach fixed-point theorem. We begin by providing suitable conditions under which $\mathbf{T}$ maps a ball into itself.

Lemma 3.9. Given $r>0$, let $\mathbf{W}$ be the closed ball in $\mathbf{L}^{3}(\Omega)$ with center at the origin and radius $r$, and assume that the data satisfy

$$
\|\mathbf{g}\|_{0, \Omega}\left(\left(1+\|\mathbf{Q}\|_{0, \infty ; \Omega}\right)\left\|\phi_{\mathrm{D}}\right\|_{1 / 2, \Gamma}+\left\|\phi_{\mathrm{r}}\right\|_{0,6 ; \Omega}\right)+\left\|\mathbf{u}_{\mathrm{D}}\right\|_{1 / 2, \Gamma}+\left\|\mathbf{u}_{\mathrm{D}}\right\|_{1 / 2, \Gamma}^{2} \leq \frac{r}{C(r)},
$$

where $C(r):=C_{\mathbf{S}} \max \left\{1, C_{\widetilde{\mathbf{S}}}\right\}(1+r)$, and $C_{\mathbf{S}}$ and $C_{\widetilde{\mathbf{S}}}$ are the constants specified in Lemmas 3.6 and 3.8 , respectively. Then, there holds $\mathbf{T}(\mathbf{W}) \subseteq \mathbf{W}$.

Proof. Given $\mathbf{w} \in \mathbf{L}^{3}(\Omega)$, from the definition of $\mathbf{T}$ ( $c f .(3.23)$ ) and the a priori estimate for $\mathbf{S}$ ( $c f$. (3.31)), we first obtain

$$
\|\mathbf{T}(\mathbf{w})\|_{0,3 ; \Omega}=\|\mathbf{S}(\widetilde{\mathbf{S}}(\mathbf{w}))\|_{0,3 ; \Omega} \leq C_{\mathbf{S}}\left\{\|\mathbf{g}\|_{0, \Omega}\left(\|\widetilde{\mathbf{S}}(\mathbf{w})\|_{0,6 ; \Omega}+\left\|\phi_{\mathbf{r}}\right\|_{0,6 ; \Omega}\right)+\left\|\mathbf{u}_{\mathrm{D}}\right\|_{1 / 2, \Gamma}+\left\|\mathbf{u}_{\mathrm{D}}\right\|_{1 / 2, \Gamma}^{2}\right\} .
$$

Then, using (3.35) to bound $\|\widetilde{\mathbf{S}}(\mathbf{w})\|_{0,6 ; \Omega}$ in the foregoing inequality, noting that $\|\mathbf{w}\|_{0,3 ; \Omega} \leq r$, and performing some minor algebraic manipulations, we arrive at

$$
\|\mathbf{T}(\mathbf{w})\|_{0,3 ; \Omega} \leq C(r)\left\{\|\mathbf{g}\|_{0, \Omega}\left(\left(1+\|\mathbf{Q}\|_{0, \infty ; \Omega}\right)\left\|\phi_{\mathrm{D}}\right\|_{1 / 2, \Gamma}+\left\|\phi_{\mathbf{r}}\right\|_{0,6 ; \Omega}\right)+\left\|\mathbf{u}_{\mathrm{D}}\right\|_{1 / 2, \Gamma}+\left\|\mathbf{u}_{\mathrm{D}}\right\|_{1 / 2, \Gamma}^{2}\right\}
$$

which, thanks to the assumption (3.41), yields $\|\mathbf{T}(\mathbf{w})\|_{0,3 ; \Omega} \leq r$ and ends the proof.

We now aim to prove that the operator $\mathbf{T}$ is Lipschitz continuous, for which, according to its definition $(c f$. (3.23)), it suffices to show that both $\mathbf{S}$ and $\widetilde{\mathbf{S}}$ satisfy this property. We begin with the corresponding result for $\mathbf{S}$.

Lemma 3.10. Let $\alpha_{\mathrm{BF}}$ be given by (3.27). Then, there holds

$$
\|\mathbf{S}(\phi)-\mathbf{S}(\boldsymbol{\psi})\|_{0,3 ; \Omega} \leq \frac{1}{\alpha_{\mathrm{BF}}}\|\mathbf{g}\|_{0, \Omega}\|\boldsymbol{\phi}-\boldsymbol{\psi}\|_{0,6 ; \Omega} \quad \forall \boldsymbol{\phi}, \boldsymbol{\psi} \in \mathbf{L}^{6}(\Omega) .
$$

Proof. Given $\boldsymbol{\phi}, \boldsymbol{\psi} \in \mathbf{L}^{6}(\Omega)$, we let $(\overrightarrow{\mathbf{u}}, \boldsymbol{\sigma}):=((\mathbf{u}, \mathbf{t}), \boldsymbol{\sigma})$ and $\left(\overrightarrow{\mathbf{u}}_{0}, \boldsymbol{\sigma}_{0}\right):=\left(\left(\mathbf{u}_{0}, \mathbf{t}_{0}\right), \boldsymbol{\sigma}_{0}\right) \in \mathbf{H} \times \mathbb{H}_{0}\left(\boldsymbol{d i v}_{3 / 2} ; \Omega\right)$ be the corresponding solutions of (3.20), so that $\mathbf{u}:=\mathbf{S}(\phi)$ and $\mathbf{u}_{0}:=\mathbf{S}(\boldsymbol{\psi})$. Then, subtracting the corresponding problems from (3.20), we obtain

$$
\begin{aligned}
{\left[a(\overrightarrow{\mathbf{u}})-a\left(\overrightarrow{\mathbf{u}}_{0}\right), \overrightarrow{\mathbf{v}}\right]+\left[b(\overrightarrow{\mathbf{v}}), \boldsymbol{\sigma}-\boldsymbol{\sigma}_{0}\right] } & =\left[F_{\boldsymbol{\phi}}-F_{\boldsymbol{\psi}}, \overrightarrow{\mathbf{v}}\right] & & \forall \overrightarrow{\mathbf{v}} \in \mathbf{H}, \\
{\left[b\left(\overrightarrow{\mathbf{u}}-\overrightarrow{\mathbf{u}}_{0}\right), \boldsymbol{\tau}\right] } & =0 & & \forall \boldsymbol{\tau} \in \mathbb{H}_{0}\left(\boldsymbol{d i v}_{3 / 2} ; \Omega\right) .
\end{aligned}
$$

We note from the second equation of (3.44) that $\overrightarrow{\mathbf{u}}-\overrightarrow{\mathbf{u}}_{0} \in \mathbf{V}\left(c f\right.$. (3.26)). Hence, taking $\overrightarrow{\mathbf{v}}:=\overrightarrow{\mathbf{u}}-\overrightarrow{\mathbf{u}}_{0}$ in the first equation of (3.44), and applying (3.30) with $\overrightarrow{\mathbf{u}}, \overrightarrow{\mathbf{u}}_{0} \in \mathbf{H}$, we obtain

$$
\alpha_{\mathrm{BF}}\left\|\overrightarrow{\mathbf{u}}-\overrightarrow{\mathbf{u}}_{0}\right\|^{2} \leq\left[a(\overrightarrow{\mathbf{u}})-a\left(\overrightarrow{\mathbf{u}}_{0}\right), \overrightarrow{\mathbf{u}}-\overrightarrow{\mathbf{u}}_{0}\right]=\left[F_{\boldsymbol{\phi}}-F_{\boldsymbol{\psi}}, \overrightarrow{\mathbf{u}}-\overrightarrow{\mathbf{u}}_{0}\right] .
$$

In turn, recalling the definitions of $F_{\phi}(c f .(2.18))$ and $\mathbf{f}(c f .(2.2))$, employing Hölder's inequality, and using that $\varrho \geq 1$, we find that

$$
\left[F_{\boldsymbol{\phi}}-F_{\boldsymbol{\psi}}, \overrightarrow{\mathbf{u}}-\overrightarrow{\mathbf{u}}_{0}\right]=\int_{\Omega}\left\{\left(\psi_{1}-\phi_{1}\right)-\frac{1}{\varrho}\left(\psi_{2}-\phi_{2}\right)\right\} \mathbf{g} \cdot\left(\mathbf{u}-\mathbf{u}_{0}\right) \leq\|\mathbf{g}\|_{0, \Omega}\|\boldsymbol{\phi}-\boldsymbol{\psi}\|_{0,6 ; \Omega}\left\|\overrightarrow{\mathbf{u}}-\overrightarrow{\mathbf{u}}_{0}\right\|,
$$

which, replaced back into (3.45), yields (3.43) and completes the proof. 
We now establish the Lipschitz-continuity of $\widetilde{\mathbf{S}}$.

Lemma 3.11. There exists a positive constant $L_{\widetilde{\mathbf{S}}}$, depending on $\mathrm{R}_{j}, \widetilde{\alpha}_{j}$, and $\widetilde{\beta}, j \in\{1,2\}$, such that

$$
\|\widetilde{\mathbf{S}}(\mathbf{w})-\widetilde{\mathbf{S}}(\mathbf{z})\|_{0,6 ; \Omega} \leq L_{\widetilde{\mathbf{S}}}\left(1+\|\mathbf{Q}\|_{0, \infty ; \Omega}+\|\mathbf{w}\|_{0,3 ; \Omega}\right)\left\|\phi_{\mathrm{D}}\right\|_{1 / 2, \Gamma}\|\mathbf{w}-\mathbf{z}\|_{0,3 ; \Omega}
$$

for all $\mathbf{w}, \mathbf{z} \in \mathbf{L}^{3}(\Omega)$.

Proof. We proceed similarly to Lemma 3.8 of [18]. In fact, given $\mathbf{w}, \mathbf{z} \in \mathbf{L}^{3}(\Omega)$, for each $j \in\{1,2\}$ we let $\left(\vec{\phi}_{j}, \boldsymbol{\rho}_{j}\right):=\left(\left(\phi_{j}, \widetilde{\mathbf{t}}_{j}\right), \boldsymbol{\rho}_{j}\right),\left(\vec{\varphi}_{j}, \boldsymbol{\xi}_{j}\right):=\left(\left(\varphi_{j}, \widetilde{\mathbf{s}}_{j}\right), \boldsymbol{\xi}_{j}\right) \in \widetilde{\mathbf{H}} \times \mathbf{H}\left(\operatorname{div}_{6 / 5} ; \Omega\right)$ be the respective solutions of (3.22), so that $\left(\phi_{1}, \phi_{2}\right)=\left(\widetilde{\mathbf{S}}_{1}(\mathbf{w}), \widetilde{\mathbf{S}}_{2}(\mathbf{w})\right)=\widetilde{\mathbf{S}}(\mathbf{w})$ and $\left(\varphi_{1}, \varphi_{2}\right)=\left(\widetilde{\mathbf{S}}_{1}(\mathbf{z}), \widetilde{\mathbf{S}}_{2}(\mathbf{z})\right)=\widetilde{\mathbf{S}}(\mathbf{z})$. It follows from the corresponding second equations of (3.22) that $\vec{\phi}_{j}-\vec{\varphi}_{j} \in \tilde{\mathbf{V}}\left(c f\right.$. (3.33)), and then the $\widetilde{\mathbf{V}}$-ellipticity of $\widetilde{a}_{j}(c f .(3.34))$ and the first equations of (3.22) applied to both $\widetilde{\mathbf{S}}_{j}(\mathbf{w})$ and $\widetilde{\mathbf{S}}_{j}(\mathbf{z})$, yield

$$
\widetilde{\alpha}_{j}\left\|\vec{\phi}_{j}-\vec{\varphi}_{j}\right\|^{2} \leq\left[\widetilde{a}_{j}\left(\vec{\phi}_{j}\right)-\widetilde{a}_{j}\left(\vec{\varphi}_{j}\right), \vec{\phi}_{j}-\vec{\varphi}_{j}\right]=-\left[c_{j}(\mathbf{w})\left(\vec{\phi}_{j}\right)-c_{j}(\mathbf{z})\left(\vec{\varphi}_{j}\right), \vec{\phi}_{j}-\vec{\varphi}_{j}\right] .
$$

In turn, adding and subtracting $\left[c_{j}(\mathbf{z})\left(\vec{\phi}_{j}\right), \vec{\phi}_{j}-\vec{\varphi}_{j}\right]$, and using the properties (3.17) and (3.18) satisfied by $c_{j}$, we deduce from the foregoing inequality that

$$
\begin{aligned}
\widetilde{\alpha}_{j}\left\|\vec{\phi}_{j}-\vec{\varphi}_{j}\right\|^{2} & \leq-\left[c_{j}(\mathbf{w}-\mathbf{z})\left(\vec{\phi}_{j}\right), \vec{\phi}_{j}-\vec{\varphi}_{j}\right]-\left[c_{j}(\mathbf{z})\left(\vec{\phi}_{j}-\vec{\varphi}_{j}\right), \vec{\phi}_{j}-\vec{\varphi}_{j}\right] \\
& \leq \mathrm{R}_{j}\left\|\vec{\phi}_{j}\right\|\|\mathbf{w}-\mathbf{z}\|_{0,3 ; \Omega}\left\|\vec{\phi}_{j}-\vec{\varphi}_{j}\right\|,
\end{aligned}
$$

which, together with the a priori estimate (3.35), implies (3.47) with $L_{\widetilde{\mathbf{S}}}:=C_{\widetilde{\mathbf{S}}} \max \left\{\widetilde{\alpha}_{1}^{-1} \mathrm{R}_{1}, \widetilde{\alpha}_{2}^{-1} R_{2}\right\}$ and concludes the proof.

As a consequence of Lemmas 3.10 and 3.11, we provide next the Lipschitz continuity of $\mathbf{T}$.

Lemma 3.12. Let us define $L_{\mathbf{T}}:=\alpha_{\mathrm{BF}}^{-1} L_{\widetilde{\mathbf{S}}}$, with $\alpha_{\mathrm{BF}}$ and $L_{\widetilde{\mathbf{S}}}$ satisfying (3.27) and (3.47), respectively. Then, there holds

$$
\|\mathbf{T}(\mathbf{w})-\mathbf{T}(\mathbf{z})\|_{0,3 ; \Omega} \leq L_{\mathbf{T}}\left(1+\|\mathbf{Q}\|_{0, \infty ; \Omega}+\|\mathbf{w}\|_{0,3 ; \Omega}\right)\|\mathbf{g}\|_{0, \Omega}\left\|\phi_{\mathrm{D}}\right\|_{1 / 2, \Gamma}\|\mathbf{w}-\mathbf{z}\|_{0,3 ; \Omega},
$$

for all $\mathbf{w}, \mathbf{z} \in \mathbf{L}^{3}(\Omega)$.

Proof. Let $\mathbf{w}, \mathbf{z} \in \mathbf{L}^{3}(\Omega)$. Then, from the definition of $\mathbf{T}$ ( $c f$. (3.23)), and Lemma 3.10 ( $c f$. (3.43)), we deduce that

$$
\|\mathbf{T}(\mathbf{w})-\mathbf{T}(\mathbf{z})\|_{0,3 ; \Omega}=\|\mathbf{S}(\widetilde{\mathbf{S}}(\mathbf{w}))-\mathbf{S}(\widetilde{\mathbf{S}}(\mathbf{z}))\|_{0,3 ; \Omega} \leq \frac{1}{\alpha_{\mathrm{BF}}}\|\mathbf{g}\|_{0, \Omega}\|\widetilde{\mathbf{S}}(\mathbf{w})-\widetilde{\mathbf{S}}(\mathbf{z})\|_{0,6 ; \Omega} .
$$

Hence, using the Lipschitz-continuity of the operator $\widetilde{\mathbf{S}}(c f .(3.47))$, we find that

$$
\|\mathbf{T}(\mathbf{w})-\mathbf{T}(\mathbf{z})\|_{0,3 ; \Omega} \leq \frac{L_{\widetilde{\mathbf{S}}}}{\alpha_{\mathrm{BF}}}\left(1+\|\mathbf{Q}\|_{0, \infty ; \Omega}+\|\mathbf{w}\|_{0,3 ; \Omega}\right)\|\mathbf{g}\|_{0, \Omega}\left\|\phi_{\mathrm{D}}\right\|_{1 / 2, \Gamma}\|\mathbf{w}-\mathbf{z}\|_{0,3 ; \Omega}
$$

which yields (3.48) and ends the proof.

We are now in position to establish the main result concerning the solvability of (2.27).

Theorem 3.13. Given $r>0$, let $\mathbf{W}$ be the closed ball in $\mathbf{L}^{3}(\Omega)$ with center at the origin and radius $r$, and assume that the data satisfy (3.41) and

$$
L_{\mathbf{T}}\left(1+\|\mathbf{Q}\|_{0, \infty ; \Omega}+r\right)\|\mathbf{g}\|_{0, \Omega}\left\|\phi_{\mathrm{D}}\right\|_{1 / 2, \Gamma}<1 .
$$


Then the operator $\mathbf{T}$ has a unique fixed point $\mathbf{u} \in \mathbf{W}$. Equivalently, the coupled problem (2.27) has a unique solution $(\overrightarrow{\mathbf{u}}, \boldsymbol{\sigma}) \in \mathbf{H} \times \mathbb{H}_{0}\left(\operatorname{div}_{3 / 2} ; \Omega\right)$ and $\left(\vec{\phi}_{j}, \boldsymbol{\rho}_{j}\right) \in \widetilde{\mathbf{H}} \times \mathbf{H}\left(\operatorname{div}_{6 / 5} ; \Omega\right), j \in\{1,2\}$, with $\mathbf{u} \in \mathbf{W}$. Moreover, there exist positive constants $C_{i}, i \in\{1,2,3,4\}$, depending on $r,|\Omega|, L_{\mathrm{BF}}, \alpha_{\mathrm{BF}}, \beta,\left\|\mathbf{Q}_{j}\right\|_{0, \infty ; \Omega}, \mathrm{R}_{j}, \widetilde{\alpha}_{j}$, and $\widetilde{\beta}$, such that the following a priori estimates hold

$$
\begin{aligned}
&\|\overrightarrow{\mathbf{u}}\| \leq C_{1}\left\{\|\mathbf{g}\|_{0, \Omega}\left(\left\|\phi_{\mathrm{D}}\right\|_{1 / 2, \Gamma}+\left\|\phi_{\mathrm{r}}\right\|_{0,6 ; \Omega}\right)+\left\|\mathbf{u}_{\mathrm{D}}\right\|_{1 / 2, \Gamma}+\left\|\mathbf{u}_{\mathrm{D}}\right\|_{1 / 2, \Gamma}^{2}\right\} \\
&\|\boldsymbol{\sigma}\|_{\operatorname{div}_{3 / 2} ; \Omega} \leq C_{2} \sum_{j=1}^{2}\left\{\left(\|\mathbf{g}\|_{0, \Omega}\left(\left\|\phi_{\mathrm{D}}\right\|_{1 / 2, \Gamma}+\left\|\phi_{\mathrm{r}}\right\|_{0,6 ; \Omega}\right)+\left\|\mathbf{u}_{\mathrm{D}}\right\|_{1 / 2, \Gamma}+\left\|\mathbf{u}_{\mathrm{D}}\right\|_{1 / 2, \Gamma}^{2}\right)^{j}\right\} \\
&\left\|\vec{\phi}_{j}\right\| \leq C_{3}\left\|\phi_{j, \mathrm{D}}\right\|_{1 / 2, \Gamma}, \quad \text { and } \\
&\left\|\boldsymbol{\rho}_{j}\right\|_{\operatorname{div}_{6 / 5} ; \Omega} \leq C_{4}\left\|\phi_{j, \mathrm{D}}\right\|_{1 / 2, \Gamma} .
\end{aligned}
$$

Proof. We begin by recalling from Lemma 3.9 that, under the assumption (3.41), $\mathbf{T}$ maps the ball $\mathbf{W}$ into itself, and hence, for each $\mathbf{w} \in \mathbf{W}$ we have that both $\|\mathbf{w}\|_{0,3 ; \Omega}$ and $\|\mathbf{T}(\mathbf{w})\|_{0,3 ; \Omega}$ are bounded by $r$. In turn, it is clear from Lemma 3.12 and Hypotheses (3.49) that $\mathbf{T}$ is a contraction. Therefore, the Banach fixed-point theorem provides the existence of a unique fixed point $\mathbf{u} \in \mathbf{W}$ of $\mathbf{T}$, equivalently, the existence of a unique solution $(\overrightarrow{\mathbf{u}}, \boldsymbol{\sigma}) \in \mathbf{H} \times \mathbb{H}_{0}\left(\operatorname{div}_{3 / 2} ; \Omega\right)$ and $\left(\vec{\phi}_{j}, \boldsymbol{\rho}_{j}\right) \in \widetilde{\mathbf{H}} \times \mathbf{H}\left(\operatorname{div}_{6 / 5} ; \Omega\right), j \in\{1,2\}$, of the coupled problem (2.27), with $\mathbf{u} \in \mathbf{W}$. In addition, it is clear that the estimates (3.52) and (3.53) follow straightforwardly from (3.39) and (3.40), respectively, whereas proceeding as in (3.42), that is, combining (3.31) (respectively (3.32)) with (3.35), we obtain (3.50) (respectively (3.51)), which finishes the proof.

\section{The Galerkin sCHeme}

In this section we introduce and analyze the corresponding Galerkin scheme for the fully-mixed formulation (2.27). The solvability of this scheme is addressed following basically the same techniques employed throughout Section 3.

\subsection{Preliminaries}

We first let $\left\{\mathcal{T}_{h}\right\}_{h>0}$ be a regular family of triangulations of $\bar{\Omega}$ by triangles $K$ (respectively tetrahedra $K$ in $\left.\mathrm{R}^{3}\right)$, and set $h:=\max \left\{h_{K}: K \in \mathcal{T}_{h}\right\}$. In turn, given an integer $l \geq 0$ and a subset $S$ of $\mathrm{R}^{n}$, we denote by $\mathrm{P}_{l}(S)$ the space of polynomials of total degree at most $l$ defined on $S$. Hence, for each integer $k \geq 0$ and for each $K \in \mathcal{T}_{h}$, we define the local Raviart-Thomas space of order $k$ as

$$
\mathbf{R T}_{k}(K):=\mathbf{P}_{k}(K) \oplus \widetilde{\mathbf{P}}_{k}(K) \mathbf{x}
$$

where $\mathbf{x}:=\left(x_{1}, \ldots, x_{n}\right)^{\mathrm{t}}$ is a generic vector of $\mathrm{R}^{n}, \widetilde{\mathrm{P}}_{k}(K)$ is the space of polynomials of total degree equal to $k$ defined on $K$, and, according to the convention in Section 1 , we set $\mathbf{P}_{k}(K):=\left[\mathrm{P}_{k}(K)\right]^{n}$ and $\mathbb{P}_{k}(K):=$ $\left[\mathrm{P}_{k}(K)\right]^{n \times n}$. In this way, introducing the finite element subspaces:

$$
\begin{aligned}
\mathbf{H}_{h}^{\mathbf{u}} & :=\left\{\mathbf{v}_{h} \in \mathbf{L}^{3}(\Omega):\left.\mathbf{v}_{h}\right|_{K} \in \mathbf{P}_{k}(K) \quad \forall K \in \mathcal{T}_{h}\right\}, \\
\mathbb{H}_{h}^{\mathbf{t}} & :=\left\{\mathbf{r}_{h} \in \mathbb{L}_{\mathrm{tr}}^{2}(\Omega):\left.\mathbf{r}_{h}\right|_{K} \in \mathbb{P}_{k}(K) \quad \forall K \in \mathcal{T}_{h}\right\}, \\
\mathbb{H}_{h}^{\sigma} & :=\left\{\boldsymbol{\tau}_{h} \in \mathbb{H}_{0}\left(\operatorname{div}_{3 / 2} ; \Omega\right):\left.\mathbf{c}^{\mathrm{t}} \boldsymbol{\tau}_{h}\right|_{K} \in \mathbf{R T}_{k}(K) \quad \forall \mathbf{c} \in \mathrm{R}^{n}, \quad \forall K \in \mathcal{T}_{h}\right\}, \\
\mathrm{H}_{h}^{\phi} & :=\left\{\psi_{h} \in \mathrm{L}^{6}(\Omega):\left.\psi_{h}\right|_{K} \in \mathrm{P}_{k}(K) \quad \forall K \in \mathcal{T}_{h}\right\}, \\
\mathbf{H}_{h}^{\widetilde{\mathbf{t}}} & :=\left\{\widetilde{\mathbf{r}}_{h} \in \mathbf{L}^{2}(\Omega):\left.\widetilde{\mathbf{r}}_{h}\right|_{K} \in \mathbf{P}_{k}(K) \quad \forall K \in \mathcal{T}_{h}\right\}, \\
\mathbf{H}_{h}^{\rho} & :=\left\{\boldsymbol{\eta}_{h} \in \mathbf{H}\left(\operatorname{div}_{6 / 5} ; \Omega\right):\left.\boldsymbol{\eta}_{h}\right|_{K} \in \mathbf{R} \mathbf{T}_{k}(K) \quad \forall K \in \mathcal{T}_{h}\right\},
\end{aligned}
$$


and denoting from now on $\phi_{h}:=\left(\phi_{1, h}, \phi_{2, h}\right), \varphi_{h}:=\left(\varphi_{1, h}, \varphi_{2, h}\right) \in \mathbf{H}_{h}^{\phi}:=\mathrm{H}_{h}^{\phi} \times \mathrm{H}_{h}^{\phi}$, and

$$
\begin{array}{r}
\overrightarrow{\mathbf{u}}_{h}:=\left(\mathbf{u}_{h}, \mathbf{t}_{h}\right), \quad \overrightarrow{\mathbf{v}}_{h}:=\left(\mathbf{v}_{h}, \mathbf{r}_{h}\right), \quad \overrightarrow{\mathbf{u}}_{0, h}:=\left(\mathbf{u}_{0, h}, \mathbf{t}_{0, h}\right) \in \mathbf{H}_{h}:=\mathbf{H}_{h}^{\mathbf{u}} \times \mathbb{H}_{h}^{\mathbf{t}}, \\
\vec{\phi}_{j, h}:=\left(\phi_{j, h}, \widetilde{\mathbf{t}}_{j, h}\right), \quad \vec{\psi}_{j, h}:=\left(\psi_{j, h}, \widetilde{\mathbf{r}}_{j, h}\right) \in \widetilde{\mathbf{H}}_{h}:=\mathrm{H}_{h}^{\phi} \times \mathbf{H}_{h}^{\mathfrak{t}},
\end{array}
$$

the Galerkin scheme for (2.27) reads: Find $\left(\overrightarrow{\mathbf{u}}_{h}, \boldsymbol{\sigma}_{h}\right) \in \mathbf{H}_{h} \times \mathbb{H}_{h}^{\boldsymbol{\sigma}}$ and $\left(\vec{\phi}_{j, h}, \boldsymbol{\rho}_{j, h}\right) \in \widetilde{\mathbf{H}}_{h} \times \mathbf{H}_{h}^{\boldsymbol{\rho}}, j \in\{1,2\}$, such that

$$
\begin{aligned}
{\left[a\left(\overrightarrow{\mathbf{u}}_{h}\right), \overrightarrow{\mathbf{v}}_{h}\right]+\left[b\left(\overrightarrow{\mathbf{v}}_{h}\right), \boldsymbol{\sigma}_{h}\right] } & =\left[F_{\boldsymbol{\phi}_{h}}, \overrightarrow{\mathbf{v}}_{h}\right] & & \forall \overrightarrow{\mathbf{v}}_{h} \in \mathbf{H}_{h}, \\
{\left[b\left(\overrightarrow{\mathbf{u}}_{h}\right), \boldsymbol{\tau}_{h}\right] } & =\left[G_{\mathrm{D}}, \boldsymbol{\tau}_{h}\right] & & \forall \boldsymbol{\tau}_{h} \in \mathbb{H}_{h}^{\boldsymbol{\sigma}}, \\
{\left[\widetilde{a}_{j}\left(\vec{\phi}_{j, h}\right), \vec{\psi}_{j, h}\right]+\left[c_{j}\left(\mathbf{u}_{h}\right)\left(\vec{\phi}_{j, h}\right), \vec{\psi}_{j, h}\right]+\left[\widetilde{b}\left(\vec{\psi}_{j, h}\right), \boldsymbol{\rho}_{j, h}\right] } & =0 & & \forall \vec{\psi}_{j, h} \in \widetilde{\mathbf{H}}_{h}, \\
{\left[\widetilde{b}\left(\vec{\phi}_{j, h}\right), \boldsymbol{\eta}_{j, h}\right] } & =\left[\widetilde{G}_{j}, \boldsymbol{\eta}_{j . h}\right] & & \forall \boldsymbol{\eta}_{j, h} \in \mathbf{H}_{h}^{\rho} .
\end{aligned}
$$

We now develop the discrete analogue of the fixed-point approach utilized in Section 3.2. To this end, we first consider the operator $\mathbf{S}_{h}: \mathbf{H}_{h}^{\phi} \rightarrow \mathbf{H}_{h}^{\mathbf{u}}$ defined by

$$
\mathbf{S}_{h}\left(\boldsymbol{\varphi}_{h}\right):=\mathbf{u}_{h} \quad \forall \boldsymbol{\varphi}_{h} \in \mathbf{H}_{h}^{\phi}
$$

where $\left(\overrightarrow{\mathbf{u}}_{h}, \boldsymbol{\sigma}_{h}\right):=\left(\left(\mathbf{u}_{h}, \mathbf{t}_{h}\right), \boldsymbol{\sigma}_{h}\right) \in \mathbf{H}_{h} \times \mathbb{H}_{h}^{\sigma}$ is the unique solution (to be confirmed below) of the first two equations of (4.2) with the given $\boldsymbol{\varphi}_{h} \in \mathbf{H}_{h}^{\phi}$ in place of $\boldsymbol{\phi}_{h}$, that is:

$$
\begin{aligned}
{\left[a\left(\overrightarrow{\mathbf{u}}_{h}\right), \overrightarrow{\mathbf{v}}_{h}\right]+\left[b\left(\overrightarrow{\mathbf{v}}_{h}\right), \boldsymbol{\sigma}_{h}\right] } & =\left[F_{\boldsymbol{\varphi}_{h}}, \overrightarrow{\mathbf{v}}_{h}\right] & & \forall \overrightarrow{\mathbf{v}}_{h} \in \mathbf{H}_{h}, \\
{\left[b\left(\overrightarrow{\mathbf{u}}_{h}\right), \boldsymbol{\tau}_{h}\right] } & =\left[G_{\mathrm{D}}, \boldsymbol{\tau}_{h}\right] & & \forall \boldsymbol{\tau}_{h} \in \mathbb{H}_{h}^{\boldsymbol{\sigma}} .
\end{aligned}
$$

In turn, for each $j \in\{1,2\}$ we let $\widetilde{\mathbf{S}}_{j, h}: \mathbf{H}_{h}^{\mathbf{u}} \rightarrow \mathrm{H}_{h}^{\phi}$ be the operator given by

$$
\widetilde{\mathbf{S}}_{j, h}\left(\mathbf{w}_{h}\right):=\phi_{j, h} \quad \forall \mathbf{w}_{h} \in \mathbf{H}_{h}^{\mathbf{u}},
$$

where $\left(\vec{\phi}_{j, h}, \boldsymbol{\rho}_{j, h}\right):=\left(\left(\phi_{j, h}, \widetilde{\mathbf{t}}_{j, h}\right), \boldsymbol{\rho}_{j, h}\right) \in \widetilde{\mathbf{H}}_{h} \times \mathbf{H}_{h}^{\rho}$ is the unique solution (to be confirmed below) of the last two equations of (4.2) with the given $\mathbf{w}_{h} \in \mathbf{H}_{h}^{\mathbf{u}}$ in place of $\mathbf{u}_{h}$, that is:

$$
\begin{aligned}
{\left[\widetilde{a}_{j}\left(\vec{\phi}_{j, h}\right), \vec{\psi}_{j, h}\right]+\left[c_{j}\left(\mathbf{w}_{h}\right)\left(\vec{\phi}_{j, h}\right), \vec{\psi}_{j, h}\right]+\left[\widetilde{b}\left(\vec{\psi}_{j, h}\right), \boldsymbol{\rho}_{j, h}\right] } & =0 & & \forall \vec{\psi}_{j, h} \in \widetilde{\mathbf{H}}_{h}, \\
{\left[\widetilde{b}\left(\vec{\phi}_{j, h}\right), \boldsymbol{\eta}_{j, h}\right] } & =\left[\widetilde{G}_{j}, \boldsymbol{\eta}_{j, h}\right] & & \forall \boldsymbol{\eta}_{j, h} \in \mathbf{H}_{h}^{\rho} .
\end{aligned}
$$

Then, we set $\widetilde{\mathbf{S}}_{h}\left(\mathbf{w}_{h}\right):=\left(\widetilde{\mathbf{S}}_{1, h}\left(\mathbf{w}_{h}\right), \widetilde{\mathbf{S}}_{2, h}\left(\mathbf{w}_{h}\right)\right) \in \mathbf{H}_{h}^{\phi}$ for all $\mathbf{w}_{h} \in \mathbf{H}_{h}^{\mathbf{u}}$. Hence, introducing the operator $\mathbf{T}_{h}:$ $\mathbf{H}_{h}^{\mathbf{u}} \rightarrow \mathbf{H}_{h}^{\mathbf{u}}$ as

$$
\mathbf{T}_{h}\left(\mathbf{w}_{h}\right):=\mathbf{S}_{h}\left(\widetilde{\mathbf{S}}_{h}\left(\mathbf{w}_{h}\right)\right) \quad \forall \mathbf{w}_{h} \in \mathbf{H}_{h}^{\mathbf{u}},
$$

we realize that solving (4.2) is equivalent to seeking a fixed point of $\mathbf{T}_{h}$, that is: Find $\mathbf{u}_{h} \in \mathbf{H}_{h}^{\mathbf{u}}$ such that

$$
\mathbf{T}_{h}\left(\mathbf{u}_{h}\right)=\mathbf{u}_{h} .
$$

\subsection{Solvability analysis}

We begin by proving that (4.4) is well posed, or equivalently that $\mathbf{S}_{h}(c f .(4.3))$ is well defined. Indeed, we remark in advance that the respective proof, being the discrete analogue of the one of Lemma 3.6, makes use again of the abstract result given by Theorem 3.1. Hence, we first set the discrete kernel of $b$, which is given by

$$
\mathbf{V}_{h}:=\left\{\overrightarrow{\mathbf{v}}_{h}=\left(\mathbf{v}_{h}, \mathbf{r}_{h}\right) \in \mathbf{H}_{h}: \int_{\Omega} \boldsymbol{\tau}_{h}: \mathbf{r}_{h}+\int_{\Omega} \mathbf{v}_{h} \cdot \operatorname{div}\left(\boldsymbol{\tau}_{h}\right)=0 \quad \forall \boldsymbol{\tau}_{h} \in \mathbb{H}_{h}^{\sigma}\right\}
$$

Then, following the approach from Section 5 of [18], we now prove the discrete inf-sup condition for $b$ and an intermediate result that will be used to show later on the strong monotonicity of $a$ on $\mathbf{V}_{h}$. 
Lemma 4.1. There exist positive constants $\beta_{\mathrm{d}}$ and $C_{\mathrm{d}}$ such that

$$
\sup _{\substack{\overrightarrow{\mathbf{v}} \in \mathbf{H}_{h} \\ \overrightarrow{\mathbf{v}} \neq \mathbf{0}}} \frac{\left[b\left(\overrightarrow{\mathbf{v}}_{h}\right), \boldsymbol{\tau}_{h}\right]}{\left\|\overrightarrow{\mathbf{v}}_{h}\right\|} \geq \beta_{\mathrm{d}}\left\|\boldsymbol{\tau}_{h}\right\|_{\operatorname{div}_{3 / 2} ; \Omega} \quad \forall \boldsymbol{\tau}_{h} \in \mathbb{H}_{h}^{\boldsymbol{\sigma}}
$$

and

$$
\left\|\mathbf{r}_{h}\right\|_{0, \Omega} \geq C_{\mathrm{d}}\left\|\mathbf{v}_{h}\right\|_{0,3 ; \Omega} \quad \forall \overrightarrow{\mathbf{v}}_{h}=\left(\mathbf{v}_{h}, \mathbf{r}_{h}\right) \in \mathbf{V}_{h} .
$$

Proof. We proceed as in Lemma 4.2 of [5]. In fact, we begin by introducing the discrete space $Z_{0, h}$ defined by

$$
Z_{0, h}:=\left\{\boldsymbol{\tau}_{h} \in \mathbb{H}_{h}^{\boldsymbol{\sigma}}:\left[b\left(\mathbf{v}_{h}, \mathbf{0}\right), \boldsymbol{\tau}_{h}\right]=\int_{\Omega} \mathbf{v}_{h} \cdot \operatorname{div}\left(\boldsymbol{\tau}_{h}\right)=0 \quad \forall \mathbf{v}_{h} \in \mathbf{H}_{h}^{\mathbf{u}}\right\},
$$

which, using from (4.1) that $\operatorname{div}\left(\mathbb{H}_{h}^{\boldsymbol{\sigma}}\right) \subseteq \mathbf{H}_{h}^{\mathbf{u}}$, becomes

$$
Z_{0, h}=\left\{\boldsymbol{\tau}_{h} \in \mathbb{H}_{h}^{\boldsymbol{\sigma}}: \operatorname{div}\left(\boldsymbol{\tau}_{h}\right)=0 \quad \text { in } \quad \Omega\right\} .
$$

Next, by using the abstract equivalence result provided by Lemma 5.1 of [18] with the setting $X=\mathbf{H}_{h}^{\mathbf{u}}$, $Y=Y_{1}=\mathbb{H}_{h}^{\mathrm{t}}, Y_{2}=\{0\}, V=\mathbf{V}_{h}, Z=\mathbb{H}_{h}^{\sigma}$, and $Z_{0}=Z_{0, h}$, where $X, Y, Y_{1}, Y_{2}, V, Z$, and $Z_{0}$ correspond to the notations employed there, we deduce that (4.10) and (4.11) are jointly equivalent to the existence of positive constants $\beta_{1}$ and $\beta_{2}$, independent of $h$, such that there hold

$$
\sup _{\substack{\boldsymbol{\tau}_{h} \in \mathbb{H}_{h}^{\sigma} \boldsymbol{h} \\ \boldsymbol{\tau}_{h} \neq \mathbf{0}}} \frac{\left[b\left(\mathbf{v}_{h}, \mathbf{0}\right), \boldsymbol{\tau}_{h}\right]}{\left\|\boldsymbol{\tau}_{h}\right\|_{\mathbf{d i v}_{3 / 2} ; \Omega}}=\sup _{\substack{\boldsymbol{\tau}_{h} \in \mathbb{H}_{h}^{\sigma} \\ \boldsymbol{\tau}_{h} \neq \mathbf{0}}} \frac{\int_{\Omega} \mathbf{v}_{h} \cdot \operatorname{div}\left(\boldsymbol{\tau}_{h}\right)}{\left\|\boldsymbol{\tau}_{h}\right\|_{\operatorname{div}_{3 / 2} ; \Omega}} \geq \beta_{1}\left\|\mathbf{v}_{h}\right\|_{0,3 ; \Omega} \quad \forall \mathbf{v}_{h} \in \mathbf{H}_{h}^{\mathbf{u}}
$$

and

$$
\sup _{\substack{\mathbf{r}_{h} \in \mathbb{H}_{h}^{t} \\ \mathbf{r}_{h} \neq \mathbf{0}}} \frac{\left[b\left(\mathbf{0}, \mathbf{r}_{h}\right), \boldsymbol{\tau}_{h}\right]}{\left\|\mathbf{r}_{h}\right\|_{0, \Omega}}=\sup _{\substack{\mathbf{r}_{h} \in \mathbb{H}_{h}^{t} \\ \mathbf{r}_{h} \neq \mathbf{0}}} \frac{\int_{\Omega} \mathbf{r}_{h}: \boldsymbol{\tau}_{h}}{\left\|\mathbf{r}_{h}\right\|_{0, \Omega}} \geq \beta_{2}\left\|\boldsymbol{\tau}_{h}\right\|_{\operatorname{div}_{3 / 2} ; \Omega} \quad \forall \boldsymbol{\tau}_{h} \in Z_{0, h}
$$

Then, we observe that (4.12) follows from a slight adaptation of equation (5.45) from [18]. Furthermore, recalling from Lemma 2.3 of [24] that there exists a constant $c_{1}>0$, depending only on $\Omega$, such that

$$
c_{1}\|\boldsymbol{\tau}\|_{0, \Omega}^{2} \leq\left\|\boldsymbol{\tau}^{\mathrm{d}}\right\|_{0, \Omega}^{2}+\|\operatorname{div}(\boldsymbol{\tau})\|_{0, \Omega}^{2} \quad \forall \boldsymbol{\tau} \in \mathbb{H}_{0}(\operatorname{div} ; \Omega)
$$

and using the fact that $\boldsymbol{\tau}_{h}^{\mathrm{d}} \in \mathbb{H}_{h}^{\mathrm{t}}$, we easily get (4.13) with $\beta_{2}=c_{1}^{1 / 2}$.

We now establish the discrete strong monotonicity and continuity properties of $a(c f .(2.15))$.

Lemma 4.2. The family of operators $\left\{a\left(\cdot+\overrightarrow{\mathbf{z}}_{h}\right): \mathbf{V}_{h} \rightarrow \mathbf{V}_{h}^{\prime}: \overrightarrow{\mathbf{z}}_{h} \in \mathbf{H}_{h}\right\}$ is uniformly strongly monotone, that is, there exists $\alpha_{\mathrm{BF}, \mathrm{d}}>0$, such that

$$
\left[a\left(\overrightarrow{\mathbf{u}}_{h}+\overrightarrow{\mathbf{z}}_{h}\right)-a\left(\overrightarrow{\mathbf{v}}_{h}+\overrightarrow{\mathbf{z}}_{h}\right), \overrightarrow{\mathbf{u}}_{h}-\overrightarrow{\mathbf{v}}_{h}\right] \geq \alpha_{\mathrm{BF}, \mathrm{d}}\left\|\overrightarrow{\mathbf{u}}_{h}-\overrightarrow{\mathbf{v}}_{h}\right\|^{2},
$$

for all $\overrightarrow{\mathbf{z}}_{h}=\left(\mathbf{z}_{h}, \mathbf{s}_{h}\right) \in \mathbf{H}_{h}$, and for all $\overrightarrow{\mathbf{u}}_{h}=\left(\mathbf{u}_{h}, \mathbf{t}_{h}\right), \overrightarrow{\mathbf{v}}_{h}=\left(\mathbf{v}_{h}, \mathbf{r}_{h}\right) \in \mathbf{V}_{h}$ (cf. (4.9)). In addition, the operator $a: \mathbf{H}_{h} \rightarrow \mathbf{H}_{h}^{\prime}$ is continuous in the sense of (3.25), with the same constant $L_{\mathrm{BF}}$.

Proof. We follow an analogous reasoning to the proof of Lemma 3.5. In fact, let $\overrightarrow{\mathbf{z}}_{h}=\left(\mathbf{z}_{h}, \mathbf{s}_{h}\right) \in \mathbf{H}_{h}$ and $\overrightarrow{\mathbf{u}}_{h}=\left(\mathbf{u}_{h}, \mathbf{t}_{h}\right), \overrightarrow{\mathbf{v}_{h}}=\left(\mathbf{v}_{h}, \mathbf{r}_{h}\right) \in \mathbf{V}_{h}$. Then, according to the definition of $a$ (cf. (2.15)), and using (2.3) and Lemma 2.1, equation (2.1b) of [3] with $p=3$, we obtain, similarly to (3.29)

$$
\left[a\left(\overrightarrow{\mathbf{u}}_{h}+\overrightarrow{\mathbf{z}}_{h}\right)-a\left(\overrightarrow{\mathbf{v}}_{h}+\overrightarrow{\mathbf{z}}_{h}\right), \overrightarrow{\mathbf{u}}_{h}-\overrightarrow{\mathbf{v}}_{h}\right] \geq C_{\mathbf{K}}\left\|\mathbf{u}_{h}-\mathbf{v}_{h}\right\|_{0, \Omega}^{2}+c_{1}(\Omega) \mathrm{F}\left\|\mathbf{u}_{h}-\mathbf{v}_{h}\right\|_{0,3 ; \Omega}^{3}+\nu\left\|\mathbf{t}_{h}-\mathbf{r}_{h}\right\|_{0, \Omega}^{2}
$$


Next, bounding below the first and second terms on the right hand side of (4.15) by 0 , and employing the fact that $\overrightarrow{\mathbf{u}}_{h}-\overrightarrow{\mathbf{v}}_{h}:=\left(\mathbf{u}_{h}-\mathbf{v}_{h}, \mathbf{t}_{h}-\mathbf{r}_{h}\right) \in \mathbf{V}_{h}$ in combination with the estimate (4.11), we get

$$
\left[a\left(\overrightarrow{\mathbf{u}}_{h}+\overrightarrow{\mathbf{z}}_{h}\right)-a\left(\overrightarrow{\mathbf{v}}_{h}+\overrightarrow{\mathbf{z}}_{h}\right), \overrightarrow{\mathbf{u}}_{h}-\overrightarrow{\mathbf{v}}_{h}\right] \geq \frac{\nu}{2} \min \left\{1, C_{\mathrm{d}}^{2}\right\}\left\{\left\|\mathbf{u}_{h}-\mathbf{v}_{h}\right\|_{0,3 ; \Omega}^{2}+\left\|\mathbf{t}_{h}-\mathbf{r}_{h}\right\|_{0, \Omega}^{2}\right\}
$$

which gives (4.14) with $\alpha_{\mathrm{BF}, \mathrm{d}}:=\frac{\nu}{2} \min \left\{1, C_{\mathrm{d}}^{2}\right\}$. Furthermore, we now observe that for $\overrightarrow{\mathbf{u}}_{h}=\left(\mathbf{u}_{h}, \mathbf{t}_{h}\right), \overrightarrow{\mathbf{v}}_{h}=$ $\left(\mathbf{v}_{h}, \mathbf{r}_{h}\right) \in \mathbf{H}_{h}$ there certainly holds

$$
\left\|a\left(\overrightarrow{\mathbf{u}}_{h}\right)-a\left(\overrightarrow{\mathbf{v}}_{h}\right)\right\|_{\mathbf{H}_{h}^{\prime}} \leq\left\|a\left(\overrightarrow{\mathbf{u}}_{h}\right)-a\left(\overrightarrow{\mathbf{v}}_{h}\right)\right\|_{\mathbf{H}^{\prime}},
$$

whence the required continuity property of $a: \mathbf{H}_{h} \rightarrow \mathbf{H}_{h}^{\prime}$ follows directly from (3.25).

We are now in position of establishing the discrete analogue of Lemma 3.6.

Lemma 4.3. For each $\boldsymbol{\varphi}_{h} \in \mathbf{H}_{h}^{\phi}$, the problem (4.4) has a unique solution $\left(\overrightarrow{\mathbf{u}}_{h}, \boldsymbol{\sigma}_{h}\right)=\left(\left(\mathbf{u}_{h}, \mathbf{t}_{h}\right), \boldsymbol{\sigma}_{h}\right) \in \mathbf{H}_{h} \times \mathbb{H}_{h}^{\boldsymbol{\sigma}}$. Moreover, there exists a positive constant $C_{\mathbf{S}, \mathrm{d}}$, depending only on $L_{\mathrm{BF}}, \alpha_{\mathrm{BF}, \mathrm{d}}$, and $\beta_{\mathrm{d}}$, and hence independent of $\varphi_{h}$, such that

$$
\left\|\mathbf{S}_{h}\left(\boldsymbol{\varphi}_{h}\right)\right\|_{0,3 ; \Omega} \leq\left\|\overrightarrow{\mathbf{u}}_{h}\right\| \leq C_{\mathbf{S}, \mathrm{d}}\left\{\|\mathbf{g}\|_{0, \Omega}\left(\left\|\boldsymbol{\varphi}_{h}\right\|_{0,6 ; \Omega}+\left\|\boldsymbol{\phi}_{\mathbf{r}}\right\|_{0,6 ; \Omega}\right)+\left\|\mathbf{u}_{\mathrm{D}}\right\|_{1 / 2, \Gamma}+\left\|\mathbf{u}_{\mathrm{D}}\right\|_{1 / 2, \Gamma}^{2}\right\} .
$$

Proof. According to Lemma 4.2 and the discrete inf-sup condition for $b$ provided by (4.10) (cf. Lem. 4.1), the proof follows from a direct application of Theorem 3.1, with $p_{1}=3$ and $p_{2}=2$, to the discrete setting represented by (4.4). In particular, the a priori bound (4.16) is consequence of the abstract estimate (3.2) applied to (4.4), which makes use of the bounds for $G_{\mathrm{D}}$ and $F_{\boldsymbol{\varphi}_{h}}$ given by (3.12) and (3.13).

We remark here that, proceeding similarly to the derivation of (3.32), we obtain

$$
\left\|\boldsymbol{\sigma}_{h}\right\|_{\operatorname{div}_{3 / 2} ; \Omega} \leq C_{\boldsymbol{\sigma}, \mathrm{d}} \sum_{j=1}^{2}\left\{\left(\|\mathbf{g}\|_{0, \Omega}\left(\left\|\boldsymbol{\varphi}_{h}\right\|_{0,6 ; \Omega}+\left\|\phi_{\boldsymbol{r}}\right\|_{0,6 ; \Omega}\right)+\left\|\mathbf{u}_{\mathrm{D}}\right\|_{1 / 2, \Gamma}+\left\|\mathbf{u}_{\mathrm{D}}\right\|_{1 / 2, \Gamma}^{2}\right)^{j}\right\},
$$

with $C_{\boldsymbol{\sigma}, \mathrm{d}}$ depending only on $L_{\mathrm{BF}}, \alpha_{\mathrm{BF}, \mathrm{d}}$, and $\beta_{\mathrm{d}}$.

Next, we aim to show that the discrete operator $\widetilde{\mathbf{S}}_{h}$ is well defined. To this end, we now let $\widetilde{\mathbf{V}}_{h}$ be the discrete kernel of $\widetilde{b}$, that is

$$
\widetilde{\mathbf{V}}_{h}:=\left\{\vec{\psi}_{h}=\left(\psi_{h}, \widetilde{\mathbf{r}}_{h}\right) \in \widetilde{\mathbf{H}}_{h}: \int_{\Omega} \boldsymbol{\eta}_{h} \cdot \widetilde{\mathbf{r}}_{h}+\int_{\Omega} \psi_{h} \operatorname{div}\left(\boldsymbol{\eta}_{h}\right)=0 \quad \forall \boldsymbol{\eta}_{h} \in \mathbf{H}_{h}^{\rho}\right\} .
$$

Thus, we can establish a preliminary lemma, whose proof follows almost verbatim the one of Lemma 4.1 (see also [5], Lem. 4.2).

Lemma 4.4. There exist positive constants $\widetilde{\beta}_{\mathrm{d}}$ and $\widetilde{C}_{\mathrm{d}}$ such that

$$
\sup _{\substack{\vec{\psi}_{j, h} \in \widetilde{\mathbf{H}}_{h} \\ \vec{\psi}_{j, h} \neq 0}} \frac{\left[\widetilde{b}\left(\vec{\psi}_{j, h}\right), \boldsymbol{\eta}_{j, h}\right]}{\left\|\vec{\psi}_{j, h}\right\|} \geq \widetilde{\beta}_{\mathrm{d}}\left\|\boldsymbol{\eta}_{j, h}\right\|_{\operatorname{div}_{6 / 5} ; \Omega} \quad \forall \boldsymbol{\eta}_{j, h} \in \mathbf{H}_{h}^{\rho}
$$

and

$$
\left\|\widetilde{\mathbf{r}}_{j, h}\right\|_{0, \Omega} \geq \widetilde{C}_{\mathrm{d}}\left\|\psi_{j, h}\right\|_{0,6 ; \Omega} \quad \forall \vec{\psi}_{j, h}=\left(\psi_{j, h}, \widetilde{\mathbf{r}}_{j, h}\right) \in \widetilde{\mathbf{V}}_{h}
$$

The discrete analogue of Lemma 3.8 is established next. 
Lemma 4.5. For each $\mathbf{w}_{h} \in \mathbf{H}_{h}^{\mathbf{u}}$, and $j \in\{1,2\}$, problem (4.6) has a unique solution $\left(\vec{\phi}_{j, h}, \boldsymbol{\rho}_{j, h}\right)=$ $\left(\left(\phi_{j, h}, \widetilde{\mathbf{t}}_{j, h}\right), \boldsymbol{\rho}_{j, h}\right) \in \widetilde{\mathbf{H}}_{h} \times \mathbf{H}_{h}^{\rho}$. Moreover, there exists a positive constant $C_{\widetilde{\mathbf{S}}, \mathrm{d}}$, independent of $\mathbf{w}_{h}$, such that

$$
\left\|\widetilde{\mathbf{S}}_{h}\left(\mathbf{w}_{h}\right)\right\|_{0,6 ; \Omega} \leq \sum_{j=1}^{2}\left\|\vec{\phi}_{j, h}\right\| \leq C_{\widetilde{\mathbf{S}}, \mathrm{d}}\left(1+\|\mathbf{Q}\|_{0, \infty ; \Omega}+\left\|\mathbf{w}_{h}\right\|_{0,3 ; \Omega}\right)\left\|\phi_{\mathrm{D}}\right\|_{1 / 2, \Gamma}
$$

Proof. We proceed as in Lemma 3.8. In fact, given $\mathbf{w}_{h} \in \mathbf{H}_{h}^{\mathbf{u}}$, we first recall from (3.36) and (3.38) that $\mathcal{A}_{j}\left(\mathbf{w}_{h}\right)$ is bounded. Then, given $\vec{\psi}_{j, h}:=\left(\psi_{j, h}, \widetilde{\mathbf{r}}_{j, h}\right) \in \widetilde{\mathbf{V}}_{h}$, we easily deduce from (2.3), (4.19), and simple algebraic manipulations, that

$$
\left[\widetilde{a}_{j}\left(\vec{\psi}_{j, h}\right), \vec{\psi}_{j, h}\right]=\int_{\Omega} \mathbf{Q}_{j} \widetilde{\mathbf{r}}_{j, h} \cdot \widetilde{\mathbf{r}}_{j, h} \geq \widetilde{\alpha}_{j, \mathrm{~d}}\left\|\vec{\psi}_{j, h}\right\|^{2}, \quad \text { with } \quad \widetilde{\alpha}_{j, \mathrm{~d}}:=\frac{C_{\mathbf{Q}_{j}}}{2} \min \left\{1, \widetilde{C}_{\mathrm{d}}^{2}\right\}
$$

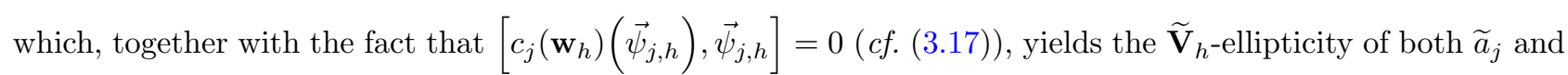
$\mathcal{A}_{j}\left(\mathbf{w}_{h}\right)$ with constant $\widetilde{\alpha}_{j, \mathrm{~d}}(c f .(4.21))$. In addition, the operator $\widetilde{b}$ satisfies the discrete inf-sup condition (4.18) ( $c f$. Lem. 4.4). Thus, we conclude by a direct application of the Babuška-Brezzi theory in Banach spaces that (4.6) is well-posed for each $j \in\{1,2\}$. In addition, the a priori estimate (4.20) follows similarly to (3.35) with $C_{\widetilde{\mathbf{S}}, \mathrm{d}}$ depending only on $\mathrm{R}_{j}, \widetilde{\alpha}_{j, \mathrm{~d}}$, and $\widetilde{\beta}_{\mathrm{d}}$.

On the other hand, we notice that, following the same arguments yielding (3.40), we are able to show that

$$
\left\|\boldsymbol{\rho}_{j, h}\right\|_{\operatorname{div}_{6 / 5} ; \Omega} \leq \frac{\widetilde{C}_{\mathrm{D}}}{\widetilde{\beta}_{\mathrm{d}}^{2}}\left(\left\|\mathbf{Q}_{j}\right\|_{0, \infty ; \Omega}+\mathrm{R}_{j}\left\|\mathbf{w}_{h}\right\|_{0,3 ; \Omega}\right)\left(1+\frac{\left\|\mathbf{Q}_{j}\right\|_{0, \infty ; \Omega}+\mathrm{R}_{j}\left\|\mathbf{w}_{h}\right\|_{0,3 ; \Omega}}{\widetilde{\alpha}_{j, \mathrm{~d}}}\right)\left\|\phi_{j, \mathrm{D}}\right\|_{1 / 2, \Gamma}
$$

In what follows we analyze the fixed-point equation (4.8). We begin with the discrete version of Lemma 3.9, whose proof, being a simple translation of the arguments proving that lemma, is omitted.

Lemma 4.6. Given $r>0$, let $\mathbf{W}_{h}$ be the closed ball in $\mathbf{H}_{h}^{\mathbf{u}}$ with center at the origin and radius $r$, and assume that the data satisfy

$$
\|\mathbf{g}\|_{0, \Omega}\left(\left(1+\|\mathbf{Q}\|_{0, \infty ; \Omega}\right)\left\|\phi_{\mathrm{D}}\right\|_{1 / 2, \Gamma}+\left\|\phi_{\boldsymbol{r}}\right\|_{0,6 ; \Omega}\right)+\left\|\mathbf{u}_{\mathrm{D}}\right\|_{1 / 2, \Gamma}+\left\|\mathbf{u}_{\mathrm{D}}\right\|_{1 / 2, \Gamma}^{2} \leq \frac{r}{C_{\mathbf{d}}(r)}
$$

where $C_{\mathrm{d}}(r):=C_{\mathbf{S}, \mathrm{d}} \max \left\{1, C_{\widetilde{\mathbf{S}}, \mathrm{d}}\right\}(1+r)$. Then $\mathbf{T}_{h}\left(\mathbf{W}_{h}\right) \subseteq \mathbf{W}_{h}$.

Next, we address the discrete counterparts of Lemmas 3.10 and 3.11, whose proofs, being almost verbatim of the continuous ones, are omitted. We just remark that Lemma 4.7 below is derived using the strong monotonicity of $a$ on $\mathbf{V}_{h}$ (cf. (4.14)), whereas the $\widetilde{\mathbf{V}}_{h}$-ellipticity of $\widetilde{a}_{j}(c f .(4.21))$ and properties (3.17) and (3.18) are employed to obtain Lemma 4.8. Thus, we simply state the corresponding results as follows.

Lemma 4.7. Let $\alpha_{\mathrm{BF}, \mathrm{d}}$ be given by (4.14). Then, there holds

$$
\left\|\mathbf{S}_{h}\left(\phi_{h}\right)-\mathbf{S}_{h}\left(\boldsymbol{\psi}_{h}\right)\right\|_{0,3 ; \Omega} \leq \frac{1}{\alpha_{\mathrm{BF}, \mathrm{d}}}\|\mathbf{g}\|_{0, \Omega}\left\|\boldsymbol{\phi}_{h}-\boldsymbol{\psi}_{h}\right\|_{0,6 ; \Omega} \quad \forall \boldsymbol{\phi}_{h}, \boldsymbol{\psi}_{h} \in \mathbf{H}_{h}^{\phi} .
$$

Lemma 4.8. There exists a positive constant $L_{\widetilde{\mathbf{S}}, \mathrm{d}}$, depending only on $\mathrm{R}_{j}, \widetilde{\alpha}_{j, \mathrm{~d}}$, and $\widetilde{\beta}_{\mathrm{d}}, j \in\{1,2\}$, such that

$$
\left\|\widetilde{\mathbf{S}}_{h}\left(\mathbf{w}_{h}\right)-\widetilde{\mathbf{S}}_{h}\left(\mathbf{z}_{h}\right)\right\|_{0,6 ; \Omega} \leq L_{\widetilde{\mathbf{S}, d}}\left(1+\|\mathbf{Q}\|_{0, \infty ; \Omega}+\left\|\mathbf{w}_{h}\right\|_{0,3 ; \Omega}\right)\left\|\phi_{\mathrm{D}}\right\|_{1 / 2, \Gamma}\left\|\mathbf{w}_{h}-\mathbf{z}_{h}\right\|_{0,3 ; \Omega}
$$

for all $\mathbf{w}_{h}, \mathbf{z}_{h} \in \mathbf{H}_{h}^{\mathbf{u}}$. 
As a straightforward consequence of Lemmas 4.7 and 4.8, we now state the Lipschitz-continuity of the operator $\mathbf{T}_{h}$ (cf. Lem. 3.12).

Lemma 4.9. Let us define $L_{\mathbf{T}, \mathrm{d}}:=\alpha_{\mathrm{BF}, \mathrm{d}}^{-1} L_{\widetilde{\mathbf{S}}, \mathrm{d}}$, with $\alpha_{\mathrm{BF}, \mathrm{d}}$ and $L_{\widetilde{\mathbf{S}}, \mathrm{d}}$ satisfying (4.14) and (4.24), respectively. Then, there holds

$$
\left\|\mathbf{T}_{h}\left(\mathbf{w}_{h}\right)-\mathbf{T}_{h}\left(\mathbf{z}_{h}\right)\right\|_{0,3 ; \Omega} \leq L_{\mathbf{T}, \mathrm{d}}\left(1+\|\mathbf{Q}\|_{0, \infty ; \Omega}+\left\|\mathbf{w}_{h}\right\|_{0,3 ; \Omega}\right)\|\mathbf{g}\|_{0, \Omega}\left\|\phi_{\mathrm{D}}\right\|_{1 / 2, \Gamma}\left\|\mathbf{w}_{h}-\mathbf{z}_{h}\right\|_{0,3 ; \Omega}
$$

for all $\mathbf{w}_{h}, \mathbf{z}_{h} \in \mathbf{H}_{h}^{\mathbf{u}}$.

We are now in position of establishing the well-posedness of (4.2).

Theorem 4.10. Given $r>0$, let $\mathbf{W}_{h}$ be the closed ball in $\mathbf{H}_{h}^{\mathbf{u}}$ with center at the origin and radius $r$, and assume that the data satisfy (4.23) and

$$
L_{\mathbf{T}, \mathrm{d}}\left(1+\|\mathbf{Q}\|_{0, \infty ; \Omega}+r\right)\|\mathbf{g}\|_{0, \Omega}\left\|\phi_{\mathrm{D}}\right\|_{1 / 2, \Gamma}<1 .
$$

Then the operator $\mathbf{T}_{h}$ has a unique fixed point $\mathbf{u}_{h} \in \mathbf{W}_{h}$. Equivalently, the coupled problem (4.2) has a unique solution $\left(\overrightarrow{\mathbf{u}}_{h}, \boldsymbol{\sigma}_{h}\right) \in \mathbf{H}_{h} \times \mathbb{H}_{h}^{\boldsymbol{\sigma}}$ and $\left(\vec{\phi}_{j, h}, \boldsymbol{\rho}_{j, h}\right) \in \widetilde{\mathbf{H}}_{h} \times \mathbf{H}_{h}^{\rho}, j \in\{1,2\}$, with $\mathbf{u}_{h} \in \mathbf{W}_{h}$. Moreover, there exist positive constants $C_{i, \mathrm{~d}}, i \in\{1,2,3,4\}$, depending on $r,|\Omega|, L_{\mathrm{BF}}, \alpha_{\mathrm{BF}, \mathrm{d}}, \beta_{\mathrm{d}},\left\|\mathbf{Q}_{j}\right\|_{0, \infty ; \Omega}, \mathrm{R}_{j}, \widetilde{\alpha}_{j, \mathrm{~d}}$, and $\widetilde{\beta}_{\mathrm{d}}$, such that the following a priori estimates hold

$$
\begin{aligned}
\left\|\overrightarrow{\mathbf{u}}_{h}\right\| & \leq C_{1, \mathrm{~d}}\left\{\|\mathbf{g}\|_{0, \Omega}\left(\left\|\phi_{\mathrm{D}}\right\|_{1 / 2, \Gamma}+\left\|\phi_{\mathrm{r}}\right\|_{0,6 ; \Omega}\right)+\left\|\mathbf{u}_{\mathrm{D}}\right\|_{1 / 2, \Gamma}+\left\|\mathbf{u}_{\mathrm{D}}\right\|_{1 / 2, \Gamma}^{2}\right\} \\
\left\|\boldsymbol{\sigma}_{h}\right\|_{\operatorname{div}_{3 / 2} ; \Omega} & \leq C_{2, \mathrm{~d}} \sum_{j=1}^{2}\left\{\left(\|\mathbf{g}\|_{0, \Omega}\left(\left\|\phi_{\mathrm{D}}\right\|_{1 / 2, \Gamma}+\left\|\phi_{\mathrm{r}}\right\|_{0,6 ; \Omega}\right)+\left\|\mathbf{u}_{\mathrm{D}}\right\|_{1 / 2, \Gamma}+\left\|\mathbf{u}_{\mathrm{D}}\right\|_{1 / 2, \Gamma}^{2}\right)^{j}\right\} \\
\left\|\vec{\phi}_{j, h}\right\| & \leq C_{3, \mathrm{~d}}\left\|\phi_{j, \mathrm{D}}\right\|_{1 / 2, \Gamma}, \quad \text { and } \\
\left\|\boldsymbol{\rho}_{j, h}\right\|_{\operatorname{div}_{6 / 5} ; \Omega} & \leq C_{4, \mathrm{~d}}\left\|\phi_{j, \mathrm{D}}\right\|_{1 / 2, \Gamma} .
\end{aligned}
$$

Proof. It follows similarly to the proof of Theorem 3.13. Indeed, we first notice from Lemma 4.6 that $\mathbf{T}_{h}$ maps the ball $\mathbf{W}_{h}$ into itself. Next, it is easy to see from (4.25) (cf. Lem. 4.9) and (4.26) that $\mathbf{T}_{h}$ is a contraction, and hence the existence and uniqueness results follow from the Banach fixed-point theorem. In addition, it is clear that the estimates (4.29) and (4.30) follow straightforwardly from (4.20) and (4.22), respectively, whereas combining (4.16) (respectively (4.17)) with (4.20) we obtain (4.27) (respectively (4.28)), which ends the proof.

\section{A PRIORI ERROR ANALYSIS}

In this section we derive the Céa estimate for the Galerkin scheme (4.2) with the finite element subspaces given by (4.1) ( $c f$. Sect. 4.1), and then use the approximation properties of the latter to establish the corresponding rates of convergence. In fact, let $(\overrightarrow{\mathbf{u}}, \boldsymbol{\sigma})=((\mathbf{u}, \mathbf{t}), \boldsymbol{\sigma}) \in \mathbf{H} \times \mathbb{H}_{0}\left(\operatorname{div}_{3 / 2} ; \Omega\right)$ and $\left(\vec{\phi}_{j}, \boldsymbol{\rho}_{j}\right)=\left(\left(\phi_{j}, \widetilde{\mathbf{t}}_{j}\right), \boldsymbol{\rho}_{j}\right) \in$ $\widetilde{\mathbf{H}} \times \mathbf{H}\left(\operatorname{div}_{6 / 5} ; \Omega\right), j \in\{1,2\}$, with $\mathbf{u} \in \mathbf{W}$, be the unique solution of the coupled problem (2.27), and let $\left(\overrightarrow{\mathbf{u}}_{h}, \boldsymbol{\sigma}_{h}\right)=\left(\left(\mathbf{u}_{h}, \mathbf{t}_{h}\right), \boldsymbol{\sigma}_{h}\right) \in \mathbf{H}_{h} \times \mathbb{H}_{h}^{\boldsymbol{\sigma}}$ and $\left(\vec{\phi}_{j, h}, \boldsymbol{\rho}_{j, h}\right)=\left(\left(\phi_{j, h}, \widetilde{\mathbf{t}}_{j, h}\right), \boldsymbol{\rho}_{j, h}\right) \in \widetilde{\mathbf{H}}_{h} \times \mathbf{H}_{h}^{\boldsymbol{\rho}}, j \in\{1,2\}$, with $\mathbf{u}_{h} \in \mathbf{W}_{h}$, be the unique solution of the discrete coupled problem (4.2). Then, we are interested in obtaining an a priori estimate for the global error

$$
\left\|(\overrightarrow{\mathbf{u}}, \boldsymbol{\sigma})-\left(\overrightarrow{\mathbf{u}}_{h}, \boldsymbol{\sigma}_{h}\right)\right\|+\sum_{j=1}^{2}\left\|\left(\vec{\phi}_{j}, \boldsymbol{\rho}_{j}\right)-\left(\vec{\phi}_{j, h}, \boldsymbol{\rho}_{j, h}\right)\right\| .
$$


For this purpose, we establish next an ad-hoc Strang-type estimate. Hereafter, given a subspace $X_{h}$ of a generic Banach space $\left(X,\|\cdot\|_{X}\right)$, we set as usual

$$
\operatorname{dist}\left(x, X_{h}\right):=\inf _{x_{h} \in X_{h}}\left\|x-x_{h}\right\|_{X} \text { for all } x \in X .
$$

Lemma 5.1. Let $X_{1}, X_{2}$ and $Y$ be separable and reflexive Banach spaces, being $X_{1}$ and $X_{2}$ uniformly convex, and set $X:=X_{1} \times X_{2}$. Let $\mathcal{A}: X \rightarrow X^{\prime}$ be a nonlinear operator and $\mathcal{B} \in \mathcal{L}\left(X, Y^{\prime}\right)$, such that $\mathcal{A}$ and $\mathcal{B}$ satisfy the hypotheses of Theorem 3.1 with respective constants $L, \alpha, \beta$, and exponents $p_{1}, p_{2} \geq 2$. Furthermore, let $\left\{X_{1, h}\right\}_{h>0},\left\{X_{2, h}\right\}_{h>0}$ and $\left\{Y_{h}\right\}_{h>0}$ be sequences of finite dimensional subspaces of $X_{1}, X_{2}$, and $Y$, respectively, set $X_{h}:=X_{1, h} \times X_{2, h}$, and for each $h>0$ consider a nonlinear operator $\mathcal{A}_{h}: X \rightarrow X^{\prime}$, such that $\left.\mathcal{A}_{h}\right|_{X_{h}}: X_{h} \rightarrow$ $X_{h}^{\prime}$ and $\left.\mathcal{B}\right|_{X_{h}}: X_{h} \rightarrow Y_{h}^{\prime}$ satisfy the hypotheses of Theorem 3.1 as well, with constants $L_{\mathrm{d}}, \alpha_{\mathrm{d}}$, and $\beta_{\mathrm{d}}$, all of them independent of $h$. In turn, given $\mathcal{F} \in X^{\prime}, \mathcal{G} \in Y^{\prime}$, and a sequence of functionals $\left\{\mathcal{F}_{h}\right\}_{h>0}$, with $\mathcal{F}_{h} \in X_{h}^{\prime}$ for each $h>0$, we let $(\vec{u}, \sigma)=\left(\left(u_{1}, u_{2}\right), \sigma\right) \in X \times Y$ and $\left(\vec{u}_{h}, \sigma_{h}\right)=\left(\left(u_{1, h}, u_{2, h}\right), \sigma_{h}\right) \in X_{h} \times Y_{h}$ be the unique solutions, respectively, to the problems

$$
\begin{aligned}
{[\mathcal{A}(\vec{u}), \vec{v}]+[\mathcal{B}(\vec{v}), \sigma] } & =[\mathcal{F}, \vec{v}] & & \forall \vec{v} \in X, \\
{[\mathcal{B}(\vec{u}), \tau] } & =[\mathcal{G}, \tau] & & \forall \tau \in Y,
\end{aligned}
$$

and

$$
\begin{aligned}
{\left[\mathcal{A}_{h}\left(\vec{u}_{h}\right), \vec{v}_{h}\right]+\left[\mathcal{B}\left(\vec{v}_{h}\right), \sigma_{h}\right] } & =\left[\mathcal{F}_{h}, \vec{v}_{h}\right] & & \forall \vec{v}_{h} \in X_{h}, \\
{\left[\mathcal{B}\left(\vec{u}_{h}\right), \tau_{h}\right] } & =\left[\mathcal{G}, \tau_{h}\right] & & \forall \tau_{h} \in Y_{h} .
\end{aligned}
$$

Then, there exists a positive constant $C_{\mathrm{ST}}$, depending only on $p_{1}, p_{2}, L_{\mathrm{d}}, \alpha_{\mathrm{d}}, \beta_{\mathrm{d}}$, and $\|\mathcal{B}\|$, such that

$$
\begin{aligned}
\left\|\vec{u}-\vec{u}_{h}\right\|_{X}+\left\|\sigma-\sigma_{h}\right\|_{Y} \leq & C_{\mathrm{ST}} C_{1}\left(\vec{u}, \vec{u}_{h}\right)\left\{C_{2}(\vec{u}) \operatorname{dist}\left(\vec{u}, X_{h}\right)+\sum_{j=1}^{2} \operatorname{dist}\left(\vec{u}, X_{h}\right)^{p_{j}-1}\right. \\
& \left.+\operatorname{dist}\left(\sigma, Y_{h}\right)+\left\|\mathcal{F}-\mathcal{F}_{h}\right\|_{X_{h}^{\prime}}+\left\|\mathcal{A}(\vec{u})-\mathcal{A}_{h}(\vec{u})\right\|_{X_{h}^{\prime}}\right\}
\end{aligned}
$$

where

$$
C_{1}\left(\vec{u}, \vec{u}_{h}\right):=1+\sum_{j=1}^{2}\left(\left\|u_{j}\right\|_{X_{j}}+\left\|u_{j, h}\right\|_{X_{j}}\right)^{p_{j}-2} \quad \text { and } \quad C_{2}(\vec{u}):=1+\sum_{j=1}^{2}\left\|u_{j}\right\|_{X_{j}}^{p_{j}-2} .
$$

Proof. It is basically a suitable modification of the proof of Lemma 6.1 from [18] (see also [25], Thm. B.2), which in turn, is a modification of Theorem 2.6 from [24]. We omit further details and just stress that the continuity bound and inf-sup condition of the respective linear operator $\mathcal{A}_{h}$ from Lemma 6.1 of [18] are now replaced by the corresponding continuity bound and strong monotonicity property of the present nonlinear operator $\mathcal{A}_{h}(c f$. hypotheses (i) and (ii) of Thm. 3.1), respectively.

In order to apply Lemma 5.1, we now observe that the problems (2.27) and (4.2) can be rewritten as two pairs of corresponding continuous and discrete formulations of the type defined by (5.1) and (5.2), namely

$$
\begin{aligned}
{[a(\overrightarrow{\mathbf{u}}), \overrightarrow{\mathbf{v}}]+[b(\overrightarrow{\mathbf{v}}), \boldsymbol{\sigma}] } & =\left[F_{\boldsymbol{\phi}}, \overrightarrow{\mathbf{v}}\right] & & \forall \overrightarrow{\mathbf{v}} \in \mathbf{H}, \\
{[b(\overrightarrow{\mathbf{u}}), \boldsymbol{\tau}] } & =\left[G_{\mathrm{D}}, \boldsymbol{\tau}\right] & & \forall \boldsymbol{\tau} \in \mathbb{H}_{0}\left(\operatorname{div}_{3 / 2} ; \Omega\right), \\
{\left[a\left(\overrightarrow{\mathbf{u}}_{h}\right), \overrightarrow{\mathbf{v}}_{h}\right]+\left[b\left(\overrightarrow{\mathbf{v}}_{h}\right), \boldsymbol{\sigma}_{h}\right] } & =\left[F_{\boldsymbol{\phi}_{h}}, \overrightarrow{\mathbf{v}}_{h}\right] & & \forall \overrightarrow{\mathbf{v}}_{h} \in \mathbf{H}_{h}, \\
{\left[b\left(\overrightarrow{\mathbf{u}}_{h}\right), \boldsymbol{\tau}_{h}\right] } & =\left[G_{\mathrm{D}}, \boldsymbol{\tau}_{h}\right] & & \forall \boldsymbol{\tau}_{h} \in \mathbb{H}_{h}^{\boldsymbol{\sigma}},
\end{aligned}
$$


and

$$
\begin{aligned}
{\left[\mathcal{A}_{j}(\mathbf{u})\left(\vec{\phi}_{j}\right), \vec{\psi}_{j}\right]+\left[\widetilde{b}\left(\vec{\psi}_{j}\right), \boldsymbol{\rho}_{j}\right] } & =0 & & \forall \vec{\psi}_{j} \in \widetilde{\mathbf{H}}, \\
{\left[\widetilde{b}\left(\vec{\phi}_{j}\right), \boldsymbol{\eta}_{j}\right] } & =\left[\widetilde{G}_{j}, \boldsymbol{\eta}_{j}\right] & & \forall \boldsymbol{\eta}_{j} \in \mathbf{H}\left(\operatorname{div}_{6 / 5} ; \Omega\right), \\
{\left[\mathcal{A}_{j}\left(\mathbf{u}_{h}\right)\left(\vec{\phi}_{j, h}\right), \vec{\psi}_{j, h}\right]+\left[\widetilde{b}\left(\vec{\psi}_{j, h}\right), \boldsymbol{\rho}_{j, h}\right] } & =0 & & \forall \vec{\psi}_{j, h} \in \widetilde{\mathbf{H}}_{h}, \\
{\left[\widetilde{b}\left(\vec{\phi}_{j, h}\right), \boldsymbol{\eta}_{j, h}\right] } & =\left[\widetilde{G}_{j}, \boldsymbol{\eta}_{j, h}\right] & & \forall \boldsymbol{\eta}_{j, h} \in \mathbf{H}_{h}^{\rho},
\end{aligned}
$$

where the operators $\mathcal{A}_{j}(\mathbf{u})$ and $\mathcal{A}_{j}\left(\mathbf{u}_{h}\right)$ are defined as in (3.36).

The following lemma provides a preliminary estimate for the error $\left\|(\overrightarrow{\mathbf{u}}, \boldsymbol{\sigma})-\left(\overrightarrow{\mathbf{u}}_{h}, \boldsymbol{\sigma}_{h}\right)\right\|$.

Lemma 5.2. There exists a positive constant $\widehat{C}_{\mathrm{ST}}(r)$, independent of $h$, such that

$$
\left\|(\overrightarrow{\mathbf{u}}, \boldsymbol{\sigma})-\left(\overrightarrow{\mathbf{u}}_{h}, \boldsymbol{\sigma}_{h}\right)\right\| \leq \widehat{C}_{\mathrm{ST}}(r)\left\{\operatorname{dist}\left(\overrightarrow{\mathbf{u}}, \mathbf{H}_{h}\right)+\operatorname{dist}\left(\overrightarrow{\mathbf{u}}, \mathbf{H}_{h}\right)^{2}+\operatorname{dist}\left(\boldsymbol{\sigma}, \mathbb{H}_{h}^{\boldsymbol{\sigma}}\right)+\|\mathbf{g}\|_{0, \Omega}\left\|\boldsymbol{\phi}-\boldsymbol{\phi}_{h}\right\|_{0,6 ; \Omega}\right\} .
$$

Proof. We begin by observing that the continuous and discrete systems of (5.4) satisfy the hypotheses of Theorem 3.1, with $p_{1}=3$ and $p_{2}=2$, and constants $\|b\| \leq 1, L_{\mathrm{BF}}, \alpha_{\mathrm{BF}}, \beta, \alpha_{\mathrm{BF}, \mathrm{d}}$, and $\beta_{\mathrm{d}}$ (cf. (3.9), proofs of Lems. 3.2, 3.4, 3.5, and Lems. 4.1 and 4.2). Therefore, applying Lemma 5.1 to the context given by (5.4), we deduce the existence of a constant $C_{\mathrm{ST}}>0$, depending only on $L_{\mathrm{BF}}, \alpha_{\mathrm{BF}, \mathrm{d}}$, and $\beta_{\mathrm{d}}$, such that

$$
\left\|(\overrightarrow{\mathbf{u}}, \boldsymbol{\sigma})-\left(\overrightarrow{\mathbf{u}}_{h}, \boldsymbol{\sigma}_{h}\right)\right\| \leq C_{\mathrm{ST}} C_{1}\left(\overrightarrow{\mathbf{u}}, \overrightarrow{\mathbf{u}}_{h}\right)\left\{C_{2}(\overrightarrow{\mathbf{u}}) \operatorname{dist}\left(\overrightarrow{\mathbf{u}}, \mathbf{H}_{h}\right)+\operatorname{dist}\left(\overrightarrow{\mathbf{u}}, \mathbf{H}_{h}\right)^{2}+\operatorname{dist}\left(\boldsymbol{\sigma}, \mathbb{H}_{h}^{\boldsymbol{\sigma}}\right)+\left\|F_{\boldsymbol{\phi}}-F_{\phi_{h}}\right\|_{\mathbf{H}_{h}^{\prime}}\right\} .
$$

In turn, proceeding as in (3.46), we get

$$
\left\|F_{\boldsymbol{\phi}}-F_{\phi_{h}}\right\|_{\mathbf{H}_{h}^{\prime}} \leq\|\mathbf{g}\|_{0, \Omega}\left\|\boldsymbol{\phi}-\boldsymbol{\phi}_{h}\right\|_{0,6 ; \Omega} .
$$

Finally, replacing (5.8) back into (5.7), and using the fact that $\mathbf{u} \in \mathbf{W}$ and $\mathbf{u}_{h} \in \mathbf{W}_{h}$, we readily obtain (5.6) with $\widehat{C}_{\mathrm{ST}}(r):=C_{\mathrm{ST}}(1+2 r)(1+r)$, which ends the proof.

Next, we have the following result concerning $\left\|\left(\vec{\phi}_{j}, \boldsymbol{\rho}_{j}\right)-\left(\vec{\phi}_{j, h}, \boldsymbol{\rho}_{j, h}\right)\right\|$.

Lemma 5.3. There exists a positive constant $\widetilde{C}_{\mathrm{ST}}(r)$, independent of $h$, such that

$$
\begin{aligned}
\sum_{j=1}^{2}\left\|\left(\vec{\phi}_{j}, \boldsymbol{\rho}_{j}\right)-\left(\vec{\phi}_{j, h}, \boldsymbol{\rho}_{j, h}\right)\right\| \leq \widetilde{C}_{\mathrm{ST}}(r)\left\{\sum_{j=1}^{2}\left(\operatorname{dist}\left(\vec{\phi}_{j}, \widetilde{\mathbf{H}}_{h}\right)+\operatorname{dist}\left(\boldsymbol{\rho}_{j}, \mathbf{H}_{h}^{\boldsymbol{\rho}}\right)\right)\right. \\
\left.+\left(1+\|\mathbf{Q}\|_{0, \infty ; \Omega}+r\right)\left\|\boldsymbol{\phi}_{\mathrm{D}}\right\|_{1 / 2, \Gamma}\left\|\mathbf{u}-\mathbf{u}_{h}\right\|_{0,3 ; \Omega}\right\} .
\end{aligned}
$$

Proof. It proceeds similarly to the proof of equation (6.18) from [18]. Indeed, we first observe that, with $\mathbf{u} \in \mathbf{W}$ and $\mathbf{u}_{h} \in \mathbf{W}_{h}$ given, the continuous and discrete systems of (5.5) satisfy the hypotheses of Theorem 3.1, with $p_{1}=p_{2}=2$ and constants $\|\widetilde{b}\| \leq 1, L=L_{\mathrm{d}}=\left\|\mathbf{Q}_{j}\right\|_{0, \infty ; \Omega}+\mathrm{R}_{j} r, \widetilde{\alpha}_{j}, \widetilde{\beta}, \widetilde{\alpha}_{j, \mathrm{~d}}$, and $\widetilde{\beta}_{\mathrm{d}}(c f$. (3.11), (3.38), (3.34), (3.16), (4.21), and (4.18)). Hence, applying Lemma 5.1 to the context given by (5.5), we deduce the existence of a constant $C_{\mathrm{ST}}^{j}(r)>0$, depending only on $r,\left\|\mathbf{Q}_{j}\right\|_{0, \infty ; \Omega}, \mathrm{R}_{j}, \widetilde{\alpha}_{j, \mathrm{~d}}$, and $\widetilde{\beta}_{\mathrm{d}}$, such that

$$
\left\|\left(\vec{\phi}_{j}, \boldsymbol{\rho}_{j}\right)-\left(\vec{\phi}_{j, h}, \boldsymbol{\rho}_{j, h}\right)\right\| \leq C_{\mathrm{ST}}^{j}(r)\left\{\operatorname{dist}\left(\vec{\phi}_{j}, \widetilde{\mathbf{H}}_{h}\right)+\operatorname{dist}\left(\boldsymbol{\rho}_{j}, \mathbf{H}_{h}^{\rho}\right)+\left\|\mathcal{A}_{j}(\mathbf{u})\left(\vec{\phi}_{j}\right)-\mathcal{A}_{j}\left(\mathbf{u}_{h}\right)\left(\vec{\phi}_{j}\right)\right\|_{\widetilde{\mathbf{H}}_{h}^{\prime}}\right\} .
$$


In turn, in order to bound the last term on the right-hand side of (5.10), we notice that the definition of $\mathcal{A}_{j}(\mathbf{w})$ (cf. (3.36)) and the estimate (3.18) (cf. Lem. 3.3) give

$$
\left|\left[\mathcal{A}_{j}(\mathbf{u})\left(\vec{\phi}_{j}\right)-\mathcal{A}_{j}\left(\mathbf{u}_{h}\right)\left(\vec{\phi}_{j}\right), \vec{\psi}_{j, h}\right]\right|=\left|\left[c_{j}(\mathbf{u})\left(\vec{\phi}_{j}\right)-c_{j}\left(\mathbf{u}_{h}\right)\left(\vec{\phi}_{j}\right), \vec{\psi}_{j, h}\right]\right| \leq \mathrm{R}_{j}\left\|\vec{\phi}_{j}\right\|\left\|\mathbf{u}-\mathbf{u}_{h}\right\|_{0,3 ; \Omega}\left\|\vec{\psi}_{j, h}\right\|,
$$

which, together with (5.10), the bound of $\left\|\vec{\phi}_{j}\right\|$ (cf. (3.39)), and the fact that $\mathbf{u} \in \mathbf{W}$, yields

$$
\begin{aligned}
& \left\|\left(\vec{\phi}_{j}, \boldsymbol{\rho}_{j}\right)-\left(\vec{\phi}_{j, h}, \boldsymbol{\rho}_{j, h}\right)\right\| \\
& \quad \leq C_{\mathrm{ST}}^{j}(r)\left\{\operatorname{dist}\left(\vec{\phi}_{j}, \widetilde{\mathbf{H}}_{h}\right)+\operatorname{dist}\left(\boldsymbol{\rho}_{j}, \mathbf{H}_{h}^{\rho}\right)+C_{\widetilde{\mathbf{S}}_{j}} \mathrm{R}_{j}\left(1+\left\|\mathbf{Q}_{j}\right\|_{0, \infty ; \Omega}+r\right)\left\|\phi_{j, \mathrm{D}}\right\|_{1 / 2, \Gamma}\left\|\mathbf{u}-\mathbf{u}_{h}\right\|_{0,3 ; \Omega}\right\} .
\end{aligned}
$$

The foregoing inequality leads to (5.9) with $\widetilde{C}_{\mathrm{ST}}(r):=\max \left\{\widetilde{C}_{\mathrm{ST}}^{1}(r), \widetilde{C}_{\mathrm{ST}}^{2}(r)\right\}$, where

$$
\widetilde{C}_{\mathrm{ST}}^{j}(r):=C_{\mathrm{ST}}^{j}(r) \max \left\{1, C_{\widetilde{\mathbf{S}}_{j}} \mathrm{R}_{j}\right\} \quad \forall j \in\{1,2\},
$$

thus concluding the proof.

The required Céa estimate will now follow from (5.6) and (5.9). In fact, bounding $\left\|\boldsymbol{\phi}-\boldsymbol{\phi}_{h}\right\|_{0,6 ; \Omega}$ in (5.6) by the right hand side of (5.9), we find that

$$
\begin{aligned}
\left\|(\overrightarrow{\mathbf{u}}, \boldsymbol{\sigma})-\left(\overrightarrow{\mathbf{u}}_{h}, \boldsymbol{\sigma}_{h}\right)\right\| \leq & \widehat{C}_{\mathrm{ST}}(r)\left\{\operatorname{dist}\left(\overrightarrow{\mathbf{u}}, \mathbf{H}_{h}\right)+\operatorname{dist}\left(\overrightarrow{\mathbf{u}}, \mathbf{H}_{h}\right)^{2}+\operatorname{dist}\left(\boldsymbol{\sigma}, \mathbb{H}_{h}^{\boldsymbol{\sigma}}\right)\right\} \\
& +C_{\mathrm{ST}}(r)\|\mathbf{g}\|_{0, \Omega} \sum_{j=1}^{2}\left(\operatorname{dist}\left(\vec{\phi}_{j}, \widetilde{\mathbf{H}}_{h}\right)+\operatorname{dist}\left(\boldsymbol{\rho}_{j}, \mathbf{H}_{h}^{\boldsymbol{\rho}}\right)\right) \\
& +C_{\mathrm{ST}}(r)\left(1+\|\mathbf{Q}\|_{0, \infty ; \Omega}+r\right)\|\mathbf{g}\|_{0, \Omega}\left\|\boldsymbol{\phi}_{\mathrm{D}}\right\|_{1 / 2, \Gamma}\left\|\mathbf{u}-\mathbf{u}_{h}\right\|_{0,3 ; \Omega},
\end{aligned}
$$

where $C_{\mathrm{ST}}(r):=\widehat{C}_{\mathrm{ST}}(r) \widetilde{C}_{\mathrm{ST}}(r)$. In turn, imposing the constant multiplying $\left\|\mathbf{u}-\mathbf{u}_{h}\right\|_{0,3 ; \Omega}$ in (5.11) to be sufficiently small, say $\leq 1 / 2$, we derive the a priori error estimate for $\left\|(\overrightarrow{\mathbf{u}}, \boldsymbol{\sigma})-\left(\overrightarrow{\mathbf{u}}_{h}, \boldsymbol{\sigma}_{h}\right)\right\|$. Hence, employing this latter estimate to bound the last term on the right-hand side of (5.9), we deduce the corresponding upper bound for $\sum_{j=1}^{2}\left\|\left(\vec{\phi}_{j}, \boldsymbol{\rho}_{j}\right)-\left(\vec{\phi}_{j, h}, \boldsymbol{\rho}_{j, h}\right)\right\|$. More precisely, we have proved the following result.

Theorem 5.4. Given $r>0$, assume that the datum $\phi_{\mathrm{D}}$ satisfy

$$
C_{\mathrm{ST}}(r)\left(1+\|\mathbf{Q}\|_{0, \infty ; \Omega}+r\right)\|\mathbf{g}\|_{0, \Omega}\left\|\phi_{\mathrm{D}}\right\|_{1 / 2, \Gamma} \leq \frac{1}{2}
$$

Then, there exists a positive constant $C$, independent of $h$, but depending on $r, L_{\mathrm{BF}}, \alpha_{\mathrm{BF}, \mathrm{d}}, \beta_{\mathrm{d}}, \mathrm{R}_{j}, \alpha_{j, \mathrm{~d}}, \widetilde{\beta}_{\mathrm{d}}$, $\left\|\mathbf{Q}_{j}\right\|_{0, \infty ; \Omega},\|\mathbf{g}\|_{0, \Omega}, j \in\{1,2\}$, and the datum $\boldsymbol{\phi}_{\mathrm{D}}$, such that

$$
\begin{aligned}
\left\|(\overrightarrow{\mathbf{u}}, \boldsymbol{\sigma})-\left(\overrightarrow{\mathbf{u}}_{h}, \boldsymbol{\sigma}_{h}\right)\right\| & +\sum_{j=1}^{2}\left\|\left(\vec{\phi}_{j}, \boldsymbol{\rho}_{j}\right)-\left(\vec{\phi}_{j, h}, \boldsymbol{\rho}_{j, h}\right)\right\| \\
& \leq C\left\{\operatorname{dist}\left(\overrightarrow{\mathbf{u}}, \mathbf{H}_{h}\right)+\operatorname{dist}\left(\overrightarrow{\mathbf{u}}, \mathbf{H}_{h}\right)^{2}+\operatorname{dist}\left(\boldsymbol{\sigma}, \mathbb{H}_{h}^{\boldsymbol{\sigma}}\right)+\sum_{j=1}^{2}\left(\operatorname{dist}\left(\vec{\phi}_{j}, \widetilde{\mathbf{H}}_{h}\right)+\operatorname{dist}\left(\boldsymbol{\rho}_{j}, \mathbf{H}_{h}^{\boldsymbol{\rho}}\right)\right)\right\} .
\end{aligned}
$$

At this point we remark that (3.49), (4.26), and (5.12) share a similar structure holding for a given $r>0$ and sufficiently small datum $\phi_{\mathrm{D}}$. However, these assumptions are not fully comparable since $L_{\mathbf{T}}, L_{\mathbf{T}, \mathrm{d}}$, and $C_{\mathrm{ST}}(r)$, being defined in terms of the unknown constants $\left\|i_{6}\right\|,\left\|\mathbf{i}_{3}\right\|, C_{P}, C_{\mathrm{d}}, \beta_{\mathrm{d}}$, and $\widetilde{\beta}_{\mathrm{d}}$, are not explicitly computable. As a consequence, we are not able to check in practice whether the examples to be considered 
below in Section 6 satisfy those hypotheses. Nevertheless, the numerical results reported there confirm the good performance of the method as well as the predicted rates of convergence, which suggests that the aforementioned constraints on the data are more technical issues of the analysis rather than limitations of the applicability of the numerical scheme. In addition, we stress that they do not necessarily have a physical meaning, but only constitute sufficient conditions guaranteeing that problems (2.27) and (4.2) are well-posed, and that the $a$ priori error estimate derived in Theorem 5.4 holds, respectively. In turn, it is important to highlight that (3.49), (4.26), and (5.12) are less restrictive than their counterparts for the augmented fully-mixed formulation proposed in [14], since they only require assumptions on $\phi_{\mathrm{D}}$ instead of on $\mathbf{u}_{\mathrm{D}}, \phi_{\mathrm{D}}$, and $\phi_{\mathrm{r}}$, as in equations (3.46), (4.22), and Theorem 5.4 of [14].

In order to establish the rate of convergence of the Galerkin scheme (4.2), we recall next the approximation properties of the finite element subspaces $\mathbf{H}_{h}^{\mathbf{u}}, \mathbb{H}_{h}^{\mathbf{t}}, \mathbb{H}_{h}^{\sigma}, \mathbf{H}_{h}^{\phi}, \mathbf{H}_{h}^{\widetilde{\mathbf{t}}}$, and $\mathbf{H}_{h}^{\rho}$ (cf. (4.1)), whose derivations can be found in [7,21,24,26], and Section 3.1 of [10] (see also [18], Sect. 5).

$(\mathbf{A P})_{1}$ : there exists positive constants $C_{1}, C_{2}, C_{3}$, and $C_{4}$, independent of $h$, such that for each $l \in[0, k+1]$, and for each $\mathbf{v} \in \mathbf{W}^{l, 3}(\Omega), \mathbf{r} \in \mathbb{H}^{l}(\Omega) \cap \mathbb{L}_{\mathrm{tr}}^{2}(\Omega), \psi \in \mathrm{W}^{l, 6}(\Omega)$, and $\widetilde{\mathbf{r}} \in \mathbf{H}^{l}(\Omega)$, there hold

$$
\begin{aligned}
\operatorname{dist}\left(\mathbf{v}, \mathbf{H}_{h}^{\mathbf{u}}\right) & :=\inf _{\mathbf{v}_{h} \in \mathbf{H}_{h}^{\mathbf{u}}}\left\|\mathbf{v}-\mathbf{v}_{h}\right\|_{0,3 ; \Omega} \leq C_{1} h^{l}\|\mathbf{v}\|_{l, 3 ; \Omega}, \\
\operatorname{dist}\left(\mathbf{r}, \mathbb{H}_{h}^{\mathbf{t}}\right) & :=\inf _{\mathbf{r}_{h} \in \mathbb{H}_{h}^{\mathbf{t}}}\left\|\mathbf{r}-\mathbf{r}_{h}\right\|_{0, \Omega} \leq C_{2} h^{l}\|\mathbf{r}\|_{l, \Omega}, \\
\operatorname{dist}\left(\psi, \mathrm{H}_{h}^{\phi}\right) & :=\inf _{\psi_{h} \in \mathrm{H}_{h}^{\phi}}\left\|\psi-\psi_{h}\right\|_{0,6 ; \Omega} \leq C_{3} h^{l}\|\psi\|_{l, 6 ; \Omega},
\end{aligned}
$$

and

$$
\operatorname{dist}\left(\widetilde{\mathbf{r}}, \mathbf{H}_{h}^{\widetilde{\mathbf{t}}}\right):=\inf _{\widetilde{\mathbf{r}}_{h} \in \mathbf{H}_{h}^{\widetilde{\mathbf{t}}}}\left\|\widetilde{\mathbf{r}}-\widetilde{\mathbf{r}}_{h}\right\|_{0 ; \Omega} \leq C_{4} h^{l}\|\widetilde{\mathbf{r}}\|_{l, \Omega}
$$

$(\mathbf{A P})_{2}$ : there exists positive constants $C_{5}$ and $C_{6}$, independent of $h$, such that for each $l \in(0, k+1]$, and for each $\boldsymbol{\tau} \in \mathbb{H}^{l}(\Omega) \cap \mathbb{H}_{0}\left(\operatorname{div}_{3 / 2} ; \Omega\right)$ with $\operatorname{div}(\boldsymbol{\tau}) \in \mathbf{W}^{l, 3 / 2}(\Omega)$, and $\boldsymbol{\eta} \in \mathbf{H}^{l}(\Omega) \cap \mathbf{H}\left(\operatorname{div}_{6 / 5} ; \Omega\right)$ with $\operatorname{div}(\boldsymbol{\eta}) \in \mathrm{W}^{l, 6 / 5}(\Omega)$, there hold

$$
\operatorname{dist}\left(\boldsymbol{\tau}, \mathbb{H}_{h}^{\boldsymbol{\sigma}}\right):=\inf _{\boldsymbol{\tau}_{h} \in \mathbb{H}_{h}^{\sigma}}\left\|\boldsymbol{\tau}-\boldsymbol{\tau}_{h}\right\|_{\operatorname{div}_{3 / 2} ; \Omega} \leq C_{5} h^{l}\left\{\|\boldsymbol{\tau}\|_{l, \Omega}+\|\operatorname{div}(\boldsymbol{\tau})\|_{l, 3 / 2 ; \Omega}\right\}
$$

and

$$
\operatorname{dist}\left(\boldsymbol{\eta}, \mathbf{H}_{h}^{\rho}\right):=\inf _{\boldsymbol{\eta}_{h} \in \mathbf{H}_{h}^{\rho}}\left\|\boldsymbol{\eta}-\boldsymbol{\eta}_{h}\right\|_{\operatorname{div}_{6 / 5} ; \Omega} \leq C_{6} h^{l}\left\{\|\boldsymbol{\eta}\|_{l, \Omega}+\|\operatorname{div}(\boldsymbol{\eta})\|_{l, 6 / 5 ; \Omega}\right\} .
$$

Now we are in a position to provide the theoretical rate of convergence of the Galerkin scheme (4.2).

Theorem 5.5. In addition to the hypotheses of Theorems 3.13, 4.10, and 5.4, assume that there exists $l \in$ $(0, k+1]$ such that $\mathbf{u} \in \mathbf{W}^{l, 3}(\Omega), \mathbf{t} \in \mathbb{H}^{l}(\Omega) \cap \mathbb{L}_{\mathrm{tr}}^{2}(\Omega), \boldsymbol{\sigma} \in \mathbb{H}^{l}(\Omega) \cap \mathbb{H}_{0}\left(\operatorname{div}_{3 / 2} ; \Omega\right), \operatorname{div}(\boldsymbol{\sigma}) \in \mathbf{W}^{l, 3 / 2}(\Omega)$, and for each $j \in\{1,2\}, \phi_{j} \in \mathrm{W}^{l, 6}(\Omega), \widetilde{\mathbf{t}}_{j} \in \mathbf{H}^{l}(\Omega), \boldsymbol{\rho}_{j} \in \mathbf{H}^{l}(\Omega) \cap \mathbf{H}\left(\operatorname{div}_{6 / 5} ; \Omega\right)$, and $\operatorname{div}\left(\boldsymbol{\rho}_{j}\right) \in \mathrm{W}^{l, 6 / 5}(\Omega)$. Then, there exists a positive constant $C$, independent of $h$, such that

$$
\begin{aligned}
\left\|(\overrightarrow{\mathbf{u}}, \boldsymbol{\sigma})-\left(\overrightarrow{\mathbf{u}}_{h}, \boldsymbol{\sigma}_{h}\right)\right\| & +\sum_{j=1}^{2}\left\|\left(\vec{\phi}_{j}, \boldsymbol{\rho}_{j}\right)-\left(\vec{\phi}_{j, h}, \boldsymbol{\rho}_{j, h}\right)\right\| \leq C h^{l}\left\{\|\mathbf{u}\|_{l, 3 ; \Omega}+\|\mathbf{t}\|_{l, \Omega}+\|\mathbf{u}\|_{l, 3 ; \Omega}^{2}+\|\mathbf{t}\|_{l, \Omega}^{2}\right. \\
& \left.+\|\boldsymbol{\sigma}\|_{l, \Omega}+\|\operatorname{div}(\boldsymbol{\sigma})\|_{l, 3 / 2 ; \Omega}+\sum_{j=1}^{2}\left(\left\|\phi_{j}\right\|_{l, 6 ; \Omega}+\left\|\overrightarrow{\mathbf{t}}_{j}\right\|_{l, \Omega}+\left\|\boldsymbol{\rho}_{j}\right\|_{l, \Omega}+\left\|\operatorname{div}\left(\boldsymbol{\rho}_{j}\right)\right\|_{l, 6 / 5 ; \Omega}\right)\right\} .
\end{aligned}
$$

Proof. The result follows from a direct application of Theorem 5.4 and the approximation properties provided by $(\mathbf{A P})_{1}$ and $(\mathbf{A P})_{2}$. Further details are omitted. 


\section{Numerical Results}

In this section we present three examples illustrating the performance of the fully-mixed finite element method (4.2) on a set of quasi-uniform triangulations of the respective domains, and considering the finite element subspaces defined by (4.1) (cf. Sect. 4.1). In what follows, we refer to the corresponding sets of finite element subspaces generated by $k=0$ and $k=1$, as simply $\mathbf{P}_{0}-\mathbb{P}_{0}-\mathbb{R T}_{0}-\mathrm{P}_{0}-\mathbf{P}_{0}-\mathbf{R} \mathbf{T}_{0}$ and $\mathbf{P}_{1}-\mathbb{P}_{1}-\mathbb{R T}_{1}-$ $\mathrm{P}_{1}-\mathbf{P}_{1}-\mathbf{R T}_{1}$, respectively. Our implementation is based on a FreeFem ++ code [27], in conjunction with the direct linear solver UMFPACK [20]. A Newton-Raphson algorithm with a fixed tolerance tol $=1 \mathrm{E}-6$ is used for the resolution of the nonlinear problem (4.2). As usual, the iterative method is finished when the relative error between two consecutive iterations of the complete coefficient vector, namely coeff ${ }^{m+1}$ and coeff $^{m}$, is sufficiently small, that is,

$$
\frac{\| \operatorname{coeff}^{m+1}-\text { coeff }^{m} \|}{\left\|\operatorname{coeff}^{m+1}\right\|} \leq \text { tol }
$$

where $\|\cdot\|$ stands for the usual Euclidean norm in $\mathrm{R}^{\mathrm{DOF}}$ with DOF denoting the total number of degrees of freedom defining the finite element subspaces $\mathbf{H}_{h}^{\mathbf{u}}, \mathbb{H}_{h}^{\mathbf{t}}, \mathbb{H}_{h}^{\sigma}, \mathbf{H}_{h}^{\phi}, \mathbf{H}_{h}^{\widetilde{\mathbf{t}}}$, and $\mathbf{H}_{h}^{\rho}(c f .(4.1))$.

We now introduce some additional notation. The individual errors are denoted by:

$$
\begin{gathered}
\mathrm{e}(\mathbf{u}):=\left\|\mathbf{u}-\mathbf{u}_{h}\right\|_{0,3 ; \Omega}, \quad \mathrm{e}(\mathbf{t}):=\left\|\mathbf{t}-\mathbf{t}_{h}\right\|_{0, \Omega}, \quad \mathrm{e}(\boldsymbol{\sigma}):=\left\|\boldsymbol{\sigma}-\boldsymbol{\sigma}_{h}\right\|_{\operatorname{div}_{3 / 2} ; \Omega}, \quad \mathrm{e}(p):=\left\|p-p_{h}\right\|_{0, \Omega}, \\
\mathrm{e}\left(\phi_{j}\right):=\left\|\phi_{j}-\phi_{j, h}\right\|_{0,6 ; \Omega}, \quad \mathrm{e}\left(\widetilde{\mathbf{t}}_{j}\right):=\left\|\widetilde{\mathbf{t}}_{j}-\widetilde{\mathbf{t}}_{j, h}\right\|_{0 ; \Omega}, \quad \mathrm{e}\left(\boldsymbol{\rho}_{j}\right):=\left\|\boldsymbol{\rho}_{j}-\boldsymbol{\rho}_{j, h}\right\|_{\operatorname{div}_{6 / 5} ; \Omega}, \quad j \in\{1,2\}
\end{gathered}
$$

where $p_{h}$ stands for the post-processed pressure suggested by the identity (2.7), that is

$$
p_{h}=-\frac{1}{n} \operatorname{tr}\left(\boldsymbol{\sigma}_{h}\right) .
$$

It follows that

$$
\left\|p-p_{h}\right\|_{0, \Omega}=\frac{1}{n}\left\|\operatorname{tr}\left(\boldsymbol{\sigma}-\boldsymbol{\sigma}_{h}\right)\right\|_{0, \Omega} \leq \frac{1}{\sqrt{n}}\left\|\boldsymbol{\sigma}-\boldsymbol{\sigma}_{h}\right\|_{\operatorname{div}_{3 / 2} ; \Omega},
$$

which shows that the rate of convergence for $p$ is at least the one for $\boldsymbol{\sigma}$, which is indeed confirmed below by the numerical results reported. Next, as usual, for each $\star \in\left\{\mathbf{u}, \mathbf{t}, \boldsymbol{\sigma}, p, \phi_{j}, \widetilde{\mathbf{t}}_{j}, \boldsymbol{\rho}_{j}\right\}$ we let $\mathrm{r}(\star)$ be the experimental rate of convergence given by

$$
r(\star):=\frac{\log (\mathrm{e}(\star) / \widehat{\mathrm{e}}(\star))}{\log (h / \widehat{h})},
$$

where $h$ and $\widehat{h}$ denote two consecutive meshsizes with errors e and $\widehat{e}$, respectively.

The examples to be considered in this section are described next. Similarly to Section 6 of [14], in all them we take for sake of simplicity $\nu=1, \varrho=1, \mathrm{R}_{1}=1, \mathrm{R}_{2}=1$ and $\phi_{\mathrm{r}}=(0,0)$. In turn, in the first two examples the tensors $\mathbf{K}, \mathbf{Q}_{1}$, and $\mathbf{Q}_{2}$ are taken as the identity matrix $\mathbb{I}$, which satisfy (2.3). In addition, the mean value of $\operatorname{tr}\left(\boldsymbol{\sigma}_{h}\right)$ over $\Omega$ is fixed via a Lagrange multiplier strategy (adding one row and one column to the matrix system that solves (4.4) for $\mathbf{u}_{h}, \mathbf{t}_{h}$, and $\boldsymbol{\sigma}_{h}$ ).

\section{Example 1: 2D domain with different values of the parameter F}

In this example we replicate the one from Section 6, Example 1 of [14]. More precisely, we corroborate the rates of convergence in a two-dimensional domain and also study the performance of the numerical method with respect to the number of Newton iterations required to achieve certain tolerance when different values of the parameter $\mathrm{F}$ are given. The domain is the square $\Omega=(-1,1)^{2}$. We consider the potential type gravitational acceleration $\mathbf{g}=(0,-1)^{\mathrm{t}}$, and choose the data $\mathbf{f}(c f .(2.2))$ such that the exact solution is given by 
TABLE 1. Example 1: Number of degrees of freedom, meshsizes, Newton iteration count, errors, and rates of convergence for the fully-mixed $\mathbf{P}_{0}-\mathbb{P}_{0}-\mathbb{R T}_{0}-\mathrm{P}_{0}-\mathbf{P}_{0}-\mathbf{R} \mathbf{T}_{0}$ approximation for the coupling of the Brinkman-Forchheimer and double-diffusion equations with $\mathrm{F}=10$.

\begin{tabular}{llllllllllllll}
\hline \hline DOF & $h$ & iter & $\mathrm{e}(\mathbf{u})$ & $\mathrm{r}(\mathbf{u})$ & $\mathrm{e}(\mathbf{t})$ & $\mathrm{r}(\mathbf{t})$ & $\mathrm{e}(\boldsymbol{\sigma})$ & $\mathrm{r}(\boldsymbol{\sigma})$ & $\mathrm{e}(p)$ & $\mathrm{r}(p)$ \\
\hline 644 & 0.7454 & 5 & 0.6265 & - & 3.5704 & - & 20.4886 & - & 1.7848 & - \\
2818 & 0.3667 & 5 & 0.2928 & 1.072 & 1.7526 & 1.003 & 9.1580 & 1.135 & 0.6221 & 1.486 \\
10464 & 0.1971 & 5 & 0.1527 & 1.049 & 0.9061 & 1.063 & 4.7110 & 1.071 & 0.3118 & 1.113 \\
41124 & 0.1036 & 5 & 0.0760 & 1.085 & 0.4593 & 1.057 & 2.3581 & 1.077 & 0.1521 & 1.117 \\
164698 & 0.0554 & 5 & 0.0384 & 1.087 & 0.2288 & 1.111 & 1.1832 & 1.100 & 0.0758 & 1.109 \\
665758 & 0.0284 & 5 & 0.0191 & 1.049 & 0.1130 & 1.059 & 0.5862 & 1.053 & 0.0367 & 1.088 \\
\hline $\mathrm{e}\left(\phi_{1}\right)$ & $\mathrm{r}\left(\phi_{1}\right)$ & $\mathrm{e}\left(\widetilde{\mathbf{t}}_{1}\right)$ & $\mathrm{r}\left(\widetilde{\mathbf{t}}_{1}\right)$ & $\mathrm{e}\left(\boldsymbol{\rho}_{1}\right)$ & $\mathrm{r}\left(\boldsymbol{\rho}_{1}\right)$ & $\mathrm{e}\left(\phi_{2}\right)$ & $\mathrm{r}\left(\phi_{2}\right)$ & $\mathrm{e}\left(\widetilde{\mathbf{t}}_{2}\right)$ & $\mathrm{r}\left(\widetilde{\mathbf{t}}_{2}\right)$ & $\mathrm{e}\left(\boldsymbol{\rho}_{2}\right)$ & $\mathrm{r}\left(\boldsymbol{\rho}_{2}\right)$ \\
\hline 0.0450 & - & 0.1839 & - & 0.5943 & - & 0.0759 & - & 0.2101 & - & 0.4794 & - \\
0.0227 & 0.962 & 0.1236 & 0.560 & 0.2962 & 0.982 & 0.0387 & 0.952 & 0.1023 & 1.014 & 0.2247 & 1.068 \\
0.0129 & 0.907 & 0.0712 & 0.890 & 0.1585 & 1.007 & 0.0214 & 0.950 & 0.0541 & 1.026 & 0.1148 & 1.082 \\
0.0069 & 0.977 & 0.0360 & 1.061 & 0.0796 & 1.071 & 0.0114 & 0.978 & 0.0278 & 1.040 & 0.0588 & 1.040 \\
0.0036 & 1.051 & 0.0183 & 1.080 & 0.0402 & 1.090 & 0.0062 & 0.987 & 0.0140 & 1.094 & 0.0294 & 1.105 \\
0.0018 & 1.018 & 0.0091 & 1.053 & 0.0199 & 1.055 & 0.0030 & 1.055 & 0.0069 & 1.066 & 0.0144 & 1.068 \\
\hline
\end{tabular}

$$
\begin{aligned}
\mathbf{u}\left(x_{1}, x_{2}\right) & =\left(\begin{array}{c}
\sin \left(\pi x_{1}\right) \cos \left(\pi x_{2}\right) \\
-\cos \left(\pi x_{1}\right) \sin \left(\pi x_{2}\right)
\end{array}\right), \quad p\left(x_{1}, x_{2}\right)=\cos \left(\pi x_{1}\right) \exp \left(x_{2}\right), \\
\phi_{1}\left(x_{1}, x_{2}\right) & =0.5+0.5 \cos \left(x_{1} x_{2}\right), \quad \text { and } \quad \phi_{2}\left(x_{1}, x_{2}\right)=0.1+0.3 \exp \left(x_{1} x_{2}\right) .
\end{aligned}
$$

The model problem is then complemented with the appropriate Dirichlet boundary conditions. Tables 1 and 2 show the convergence history for a sequence of quasi-uniform mesh refinements, including the number of Newton iterations when $\mathrm{F}=10$. Notice that we are able not only to approximate the original unknowns but also the pressure field through the formula (6.1). The results confirm that the optimal rates of convergence $\mathcal{O}\left(h^{k+1}\right)$ predicted by Theorem 5.5 are attained for $k=0,1$. The Newton method exhibits a behavior independent of the meshsize, converging in five iterations in all cases. In Figure 1 we display the solution obtained with the fully-mixed $\mathbf{P}_{1}-\mathbb{P}_{1}-\mathbb{R T}_{1}-\mathrm{P}_{1}-\mathbf{P}_{1}-\mathbf{R} \mathbf{T}_{1}$ approximation with meshsize $h=0.0284$ and 39102 triangle elements (actually representing $2074454 \mathrm{DOF}$ ). On the other hand, in Table 3 we show the behaviour of the iterative method as a function of the parameter $\mathrm{F} \in\left\{10^{0}, 10^{1}, 10^{2}, 10^{3}, 10^{4}, 10^{5}\right\}$, considering polynomial degree $k=0$, different meshsizes $h$, and a tolerance tol $=1 \mathrm{E}-06$. In this way, here we illustrate that the inertial term $F|\mathbf{u}| \mathbf{u}$ is well handled by the mixed finite element method (4.2), and that the latter evidences a robust behavior with respect to the parameter $F$. In fact, only 9 Newton iterations are required to converge in the most challenging case, namely $\mathrm{F}=10^{5}$.

\section{Example 2: Convergence against smooth exact solutions in a 3D domain}

We now replicate ([14], Sect. 6, Example 2). More precisely, we consider the cube domain $\Omega=(0,1)^{3}$ and the exact solution:

$$
\begin{aligned}
\mathbf{u}\left(x_{1}, x_{2}, x_{3}\right) & =\left(\begin{array}{c}
\sin \left(\pi x_{1}\right) \cos \left(\pi x_{2}\right) \cos \left(\pi x_{3}\right) \\
-2 \cos \left(\pi x_{1}\right) \sin \left(\pi x_{2}\right) \cos \left(\pi x_{3}\right) \\
\cos \left(\pi x_{1}\right) \cos \left(\pi x_{2}\right) \sin \left(\pi x_{3}\right)
\end{array}\right), \quad p\left(x_{1}, x_{2}, x_{3}\right)=\cos \left(\pi x_{1}\right) \exp \left(x_{2}+x_{3}\right), \\
\phi_{1}\left(x_{1}, x_{2}, x_{3}\right) & =0.5+0.5 \cos \left(x_{1} x_{2} x_{3}\right), \quad \text { and } \quad \phi_{2}\left(x_{1}, x_{2}, x_{3}\right)=0.1+0.3 \exp \left(x_{1} x_{2} x_{3}\right) .
\end{aligned}
$$

Similarly to the first example, we consider $\mathrm{F}=10$ and $\mathbf{g}=(0,0,-1)^{\mathrm{t}}$, whereas the data $\mathbf{f}$ is computed from $(2.2)$ using the above solution. The numerical solutions are shown in Figure 2, which were built using the fully-mixed 
TABLE 2. Example 1: Number of degrees of freedom, meshsizes, Newton iteration count, errors, and rates of convergence for the fully-mixed $\mathbf{P}_{1}-\mathbb{P}_{1}-\mathbb{R T}_{1}-\mathrm{P}_{1}-\mathbf{P}_{1}-\mathbf{R T}_{1}$ approximation for the coupling of the Brinkman-Forchheimer and double-diffusion equations with $\mathrm{F}=10$.

\begin{tabular}{llllllllllllll}
\hline \hline DOF & $h$ & iter & $\mathrm{e}(\mathbf{u})$ & $\mathrm{r}(\mathbf{u})$ & $\mathrm{e}(\mathbf{t})$ & $\mathrm{r}(\mathbf{t})$ & $\mathrm{e}(\boldsymbol{\sigma})$ & $\mathrm{r}(\boldsymbol{\sigma})$ & $\mathrm{e}(p)$ & $\mathrm{r}(p)$ \\
\hline 1972 & 0.7454 & 5 & 0.1929 & - & 0.9854 & - & 5.3894 & - & 0.3053 & - \\
8714 & 0.3667 & 5 & 0.0378 & 2.299 & 0.2021 & 2.234 & 1.1352 & 2.196 & 0.0608 & 2.274 \\
32480 & 0.1971 & 5 & 0.0100 & 2.140 & 0.0544 & 2.114 & 0.3022 & 2.132 & 0.0159 & 2.166 \\
127924 & 0.1036 & 5 & 0.0025 & 2.153 & 0.0135 & 2.172 & 0.0766 & 2.135 & 0.0039 & 2.188 \\
512898 & 0.0554 & 5 & 0.0006 & 2.217 & 0.0034 & 2.173 & 0.0191 & 2.214 & 0.0009 & 2.165 \\
2074454 & 0.0284 & 5 & 0.0001 & 2.122 & 0.0008 & 2.105 & 0.0047 & 2.116 & 0.0002 & 2.101 \\
\hline $\mathrm{e}\left(\phi_{1}\right)$ & $\mathrm{r}\left(\phi_{1}\right)$ & $\mathrm{e}\left(\widetilde{\mathbf{t}}_{1}\right)$ & $\mathrm{r}\left(\widetilde{\mathbf{t}}_{1}\right)$ & $\mathrm{e}\left(\boldsymbol{\rho}_{1}\right)$ & $\mathrm{r}\left(\boldsymbol{\rho}_{1}\right)$ & $\mathrm{e}\left(\phi_{2}\right)$ & $\mathrm{r}\left(\phi_{2}\right)$ & $\mathrm{e}\left(\widetilde{\mathbf{t}}_{2}\right)$ & $\mathrm{r}\left(\widetilde{\mathbf{t}}_{2}\right)$ & $\mathrm{e}\left(\boldsymbol{\rho}_{2}\right)$ & $\mathrm{r}\left(\boldsymbol{\rho}_{2}\right)$ \\
\hline 0.0057 & - & 0.0692 & - & 0.1702 & - & 0.0086 & - & 0.0313 & - & 0.0956 & - \\
0.0014 & 1.967 & 0.0169 & 1.990 & 0.0361 & 2.185 & 0.0022 & 1.927 & 0.0077 & 1.980 & 0.0209 & 2.143 \\
0.0004 & 1.926 & 0.0046 & 2.087 & 0.0097 & 2.125 & 0.0006 & 2.030 & 0.0022 & 1.991 & 0.0057 & 2.092 \\
0.0001 & 1.846 & 0.0011 & 2.139 & 0.0024 & 2.148 & 0.0001 & 1.888 & 0.0006 & 2.126 & 0.0015 & 2.090 \\
$3 \mathrm{E}-05$ & 2.228 & 0.0002 & 2.227 & 0.0006 & 2.214 & $5 \mathrm{E}-05$ & 2.051 & 0.0001 & 2.134 & 0.0004 & 2.182 \\
$8 \mathrm{E}-06$ & 2.113 & $7 \mathrm{E}-05$ & 2.101 & 0.0001 & 2.108 & $1 \mathrm{E}-05$ & 2.071 & $4 \mathrm{E}-05$ & 2.127 & $9 \mathrm{E}-05$ & 2.132 \\
\hline
\end{tabular}

$\mathbf{P}_{0}-\mathbb{P}_{0}-\mathbb{R T}_{0}-\mathrm{P}_{0}-\mathbf{P}_{0}-\mathbf{R T}_{0}$ approximation with meshsize $h=0.0643$ and 63888 tetrahedral elements (actually representing $1867272 \mathrm{DOF}$ ). The convergence history for a set of quasi-uniform mesh refinements using $k=0$ is shown in Table 4. Again, the mixed finite element method converges optimally with order $\mathcal{O}(h)$, as it was proved by Theorem 5.5.

\section{Example 3: Flow through porous media with channel network}

This last example is inspired by Section 5.2.4 of [2], which, similarly to Section 6, Example 3 of [14], focuses on flow through porous media with channel network. To this end, we consider the square domain $\Omega=(-1,1)^{2}$ with an internal channel network denoted as $\Omega_{\mathrm{c}}$ (see the first plot of Fig. 3 below), and boundary $\Gamma$, whose left, right, upper and lower parts are given by $\Gamma_{\text {left }}=\{-1\} \times(-1,1), \Gamma_{\text {right }}=\{1\} \times(-1,1), \Gamma_{\text {top }}=(-1,1) \times\{1\}$, and $\Gamma_{\text {bottom }}=(-1,1) \times\{-1\}$, respectively. We consider the coupling of the Brinkman-Forchheimer and doublediffusion equations (2.8) in the whole domain $\Omega$ with $\mathbf{Q}_{1}=0.5 \mathbb{I}$ and $\mathbf{Q}_{2}=0.125 \mathbb{I}$, but with different values of the parameters $\mathbf{F}$ and $\mathbf{K}=\alpha \mathbb{I}$ for the interior and the exterior of the channel, that is,

$$
\mathrm{F}=\left\{\begin{array}{ll}
10 & \text { in } \Omega_{\mathrm{c}} \\
1 & \text { in } \bar{\Omega} \backslash \Omega_{\mathrm{c}}
\end{array} \quad \text { and } \quad \alpha= \begin{cases}1 & \text { in } \Omega_{\mathrm{c}} \\
0.001 & \text { in } \bar{\Omega} \backslash \Omega_{\mathrm{c}}\end{cases}\right.
$$

The parameter choice corresponds to increased inertial effect $(\mathrm{F}=10)$ in the channel and a high permeability $(\alpha=1)$, compared to reduced inertial effect $(\mathrm{F}=1)$ in the porous medium and low permeability $(\alpha=0.001)$. In addition, the boundaries conditions are

$$
\begin{aligned}
& \mathbf{u} \cdot \mathbf{n}=0.2, \quad \mathbf{u} \cdot \mathrm{t}=0 \quad \text { on } \quad \Gamma_{\text {left }}, \quad \boldsymbol{\sigma} \mathbf{n}=\mathbf{0} \quad \text { on } \quad \Gamma \backslash \Gamma_{\text {left }}, \\
& \phi_{1}=0.3 \quad \text { on } \Gamma_{\text {bottom }}, \quad \phi_{1}=0 \quad \text { on } \Gamma_{\text {top }}, \quad \boldsymbol{\rho}_{1} \cdot \mathbf{n}=0 \quad \text { on } \quad \Gamma_{\text {left }} \cup \Gamma_{\text {right }} \text {, } \\
& \phi_{2}=0.2 \text { on } \Gamma_{\text {bottom }}, \quad \phi_{2}=0 \quad \text { on } \quad \Gamma_{\text {top }}, \quad \boldsymbol{\rho}_{2} \cdot \mathbf{n}=0 \quad \text { on } \quad \Gamma_{\text {left }} \cup \Gamma_{\text {right }} .
\end{aligned}
$$

In particular, the first row of boundary equations corresponds to inflow on the left boundary and zero stress outflow on the rest of the boundary. We point out that, differently from Section 6, Example 3 of [14], Dirichlet boundary conditions for temperature and concentration are assumed on the top and bottom of the domain instead of on the left and right sides of $\Omega$ as in [14]. We also note that, using similar arguments to those 


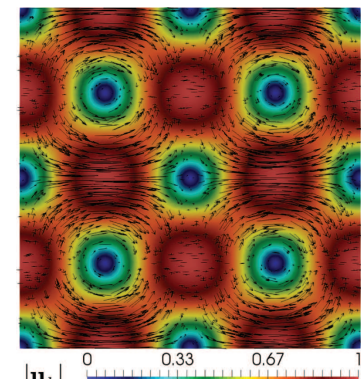

$\left|\mathbf{u}_{h}\right|$

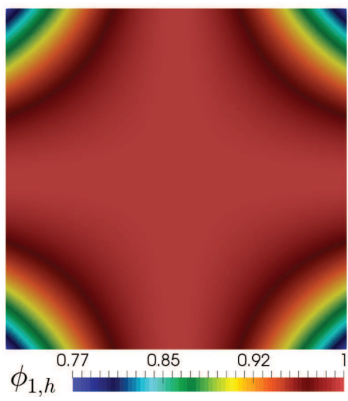

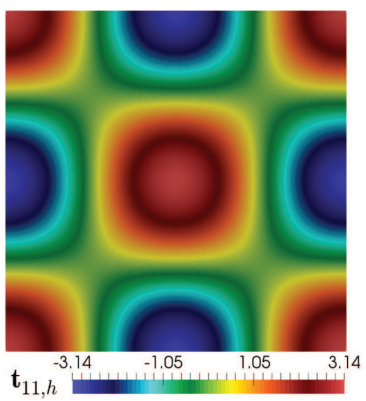

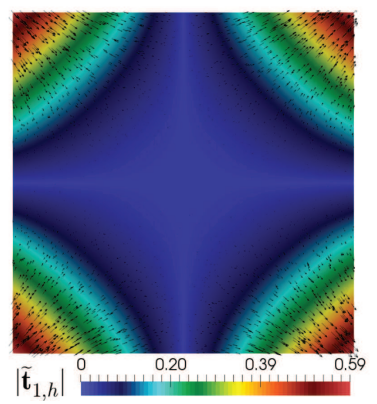

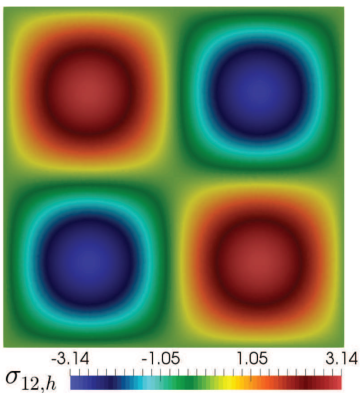
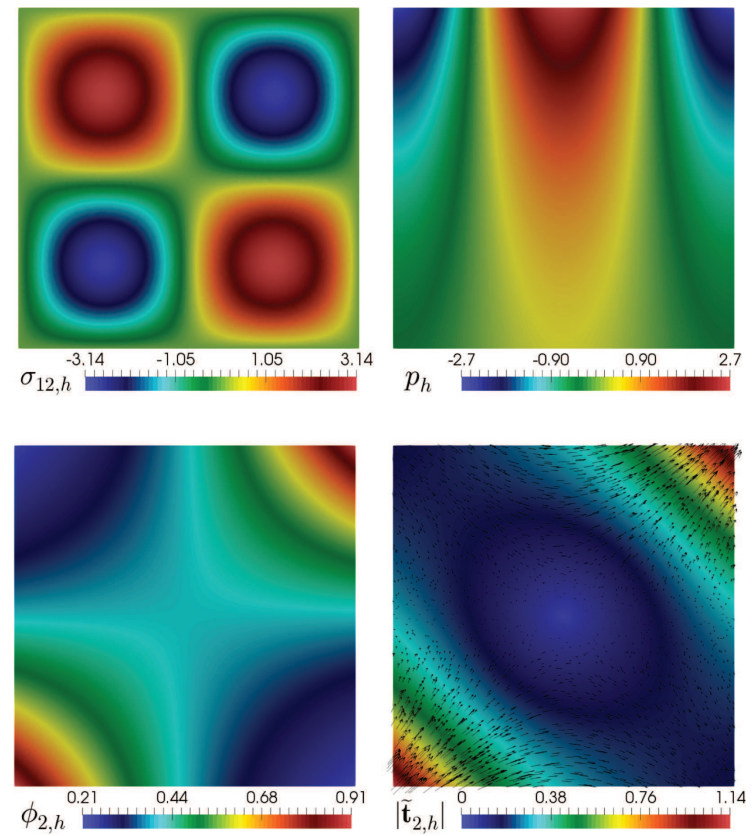

Figure 1. Example 1: Computed magnitude of the velocity, velocity gradient component, pseudostress tensor component, and pressure field (top plots); temperature field, magnitude of the temperature gradient, concentration field, and magnitude of the concentration gradient (bottom plots).

TABLE 3. Example 1: Performance of the iterative method (number of iterations) upon variations of the parameter $\mathrm{F}$ with polynomial degree $k=0$.

\begin{tabular}{lllllll}
\hline \hline & \multicolumn{6}{c}{$h$} \\
\cline { 2 - 7 }$F$ & 0.7454 & 0.3667 & 0.1971 & 0.1036 & 0.0554 & 0.0284 \\
\hline $10^{0}$ & 4 & 4 & 4 & 4 & 4 & 4 \\
$10^{1}$ & 5 & 5 & 5 & 5 & 5 & 5 \\
$10^{2}$ & 7 & 7 & 7 & 7 & 7 & 7 \\
$10^{3}$ & 8 & 8 & 8 & 8 & 8 & 8 \\
$10^{4}$ & 9 & 9 & 9 & 8 & 8 & 8 \\
$10^{5}$ & 8 & 9 & 9 & 9 & 9 & 8 \\
\hline
\end{tabular}

employed in [15], we are able to extended our analysis to the present case of mixed boundary conditions for the double-diffusion equations. In Figure 3, we display the computed magnitude of the velocity, velocity gradient, pseudostress tensor, and gradients of the temperature and concentration, and the temperature and concentration fields, which were built using the fully-mixed $\mathbf{P}_{0}-\mathbb{P}_{0}-\mathbb{R T}_{0}-\mathrm{P}_{0}-\mathbf{P}_{0}-\mathbf{R} \mathbf{T}_{0}$ approximation on a mesh with 27287 triangle elements (actually representing $475313 \mathrm{DOF}$ ). Similarly to [14], faster flow through the channel network, with a significant velocity gradient across the interface between the porous medium and the channel, are observed here. In addition, the magnitude of the pseudostress tensor is more diffused, since it includes the pressure field. In turn, the temperature and concentration are zero on the top of the domain and go increasing towards the bottom of it, which is consistent with the behavior observed in the magnitude of the temperature and concentration gradients. According to the above, we stress that the fully-mixed approach that 

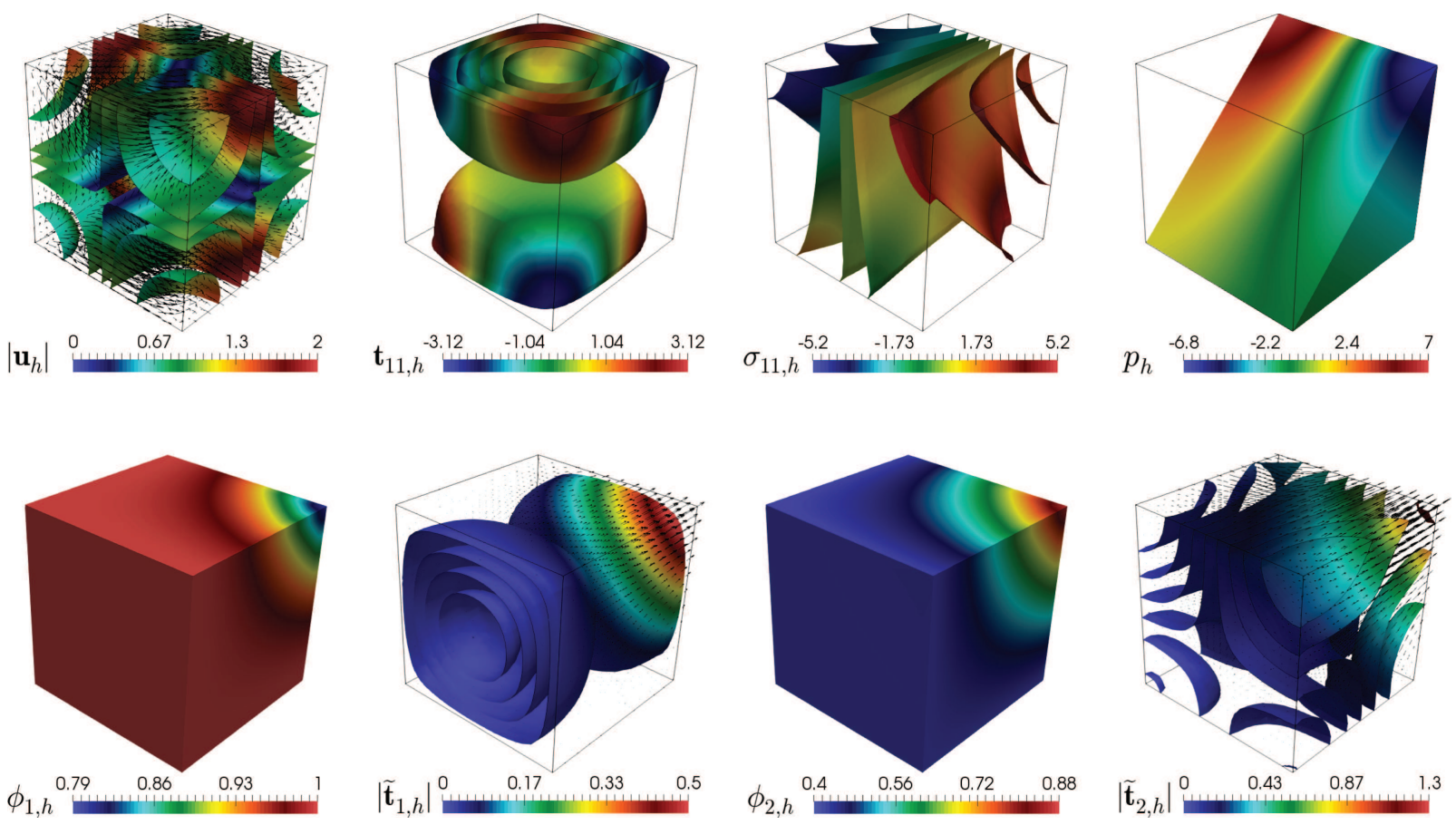

Figure 2. Example 2: Computed magnitude of the velocity, velocity gradient component, pseudostress tensor component, and pressure field (top plots); temperature field, magnitude of the temperature gradient, concentration field, and magnitude of the concentration gradient (bottom plots).

TABle 4. Example 2: Number of degrees of freedom, meshsizes, Newton iteration count, errors, and rates of convergence for the fully-mixed $\mathbf{P}_{0}-\mathbb{P}_{0}-\mathbb{R} \mathbb{T}_{0}-\mathrm{P}_{0}-\mathbf{P}_{0}-\mathbf{R} \mathbf{T}_{0}$ approximation for the coupling of the Brinkman-Forchheimer and double-diffusion equations with $\mathrm{F}=10$.

\begin{tabular}{lllllllllllllll}
\hline \hline DOF & $h$ & iter & $\mathrm{e}(\mathbf{u})$ & $\mathrm{r}(\mathbf{u})$ & $\mathrm{e}(\mathbf{t})$ & $\mathrm{r}(\mathbf{t})$ & $\mathrm{e}(\boldsymbol{\sigma})$ & $\mathrm{r}(\boldsymbol{\sigma})$ & $\mathrm{e}(p)$ & $\mathrm{r}(p)$ \\
\hline 1512 & 0.7071 & 5 & 0.5090 & - & 2.6224 & - & 15.6024 & - & 1.2501 & - \\
11616 & 0.3536 & 5 & 0.2705 & 0.912 & 1.4314 & 0.874 & 8.2301 & 0.923 & 0.6804 & 0.877 \\
91008 & 0.1768 & 5 & 0.1382 & 0.969 & 0.7391 & 0.954 & 4.1324 & 0.994 & 0.3106 & 1.131 \\
483336 & 0.1010 & 5 & 0.0793 & 0.993 & 0.4267 & 0.982 & 2.3465 & 1.011 & 0.1568 & 1.222 \\
1867272 & 0.0643 & 5 & 0.0505 & 0.998 & 0.2726 & 0.992 & 1.4870 & 1.009 & 0.0920 & 1.179 \\
\hline $\mathrm{e}\left(\phi_{1}\right)$ & $\mathrm{r}\left(\phi_{1}\right)$ & $\mathrm{e}\left(\widetilde{\mathbf{t}}_{1}\right)$ & $\mathrm{r}\left(\widetilde{\mathbf{t}}_{1}\right)$ & $\mathrm{e}\left(\boldsymbol{\rho}_{1}\right)$ & $\mathrm{r}\left(\boldsymbol{\rho}_{1}\right)$ & $\mathrm{e}\left(\phi_{2}\right)$ & $\mathrm{r}\left(\phi_{2}\right)$ & $\mathrm{e}\left(\widetilde{\mathbf{t}}_{2}\right)$ & $\mathrm{r}\left(\widetilde{\mathbf{t}}_{2}\right)$ & $\mathrm{e}\left(\boldsymbol{\rho}_{2}\right)$ & $\mathrm{r}\left(\boldsymbol{\rho}_{2}\right)$ \\
\hline 0.0379 & - & 0.0919 & - & 0.3105 & - & 0.0784 & - & 0.1062 & - & 0.2233 & - \\
0.0231 & 0.714 & 0.0793 & 0.213 & 0.1835 & 0.759 & 0.0444 & 0.820 & 0.0613 & 0.792 & 0.1229 & 0.862 \\
0.0121 & 0.937 & 0.0472 & 0.745 & 0.0972 & 0.917 & 0.0230 & 0.951 & 0.0330 & 0.896 & 0.0636 & 0.951 \\
0.0069 & 0.986 & 0.0283 & 0.913 & 0.0564 & 0.971 & 0.0132 & 0.986 & 0.0192 & 0.959 & 0.0367 & 0.983 \\
0.0044 & 0.995 & 0.0183 & 0.964 & 0.0361 & 0.988 & 0.0084 & 0.995 & 0.0124 & 0.982 & 0.0234 & 0.993 \\
\hline
\end{tabular}



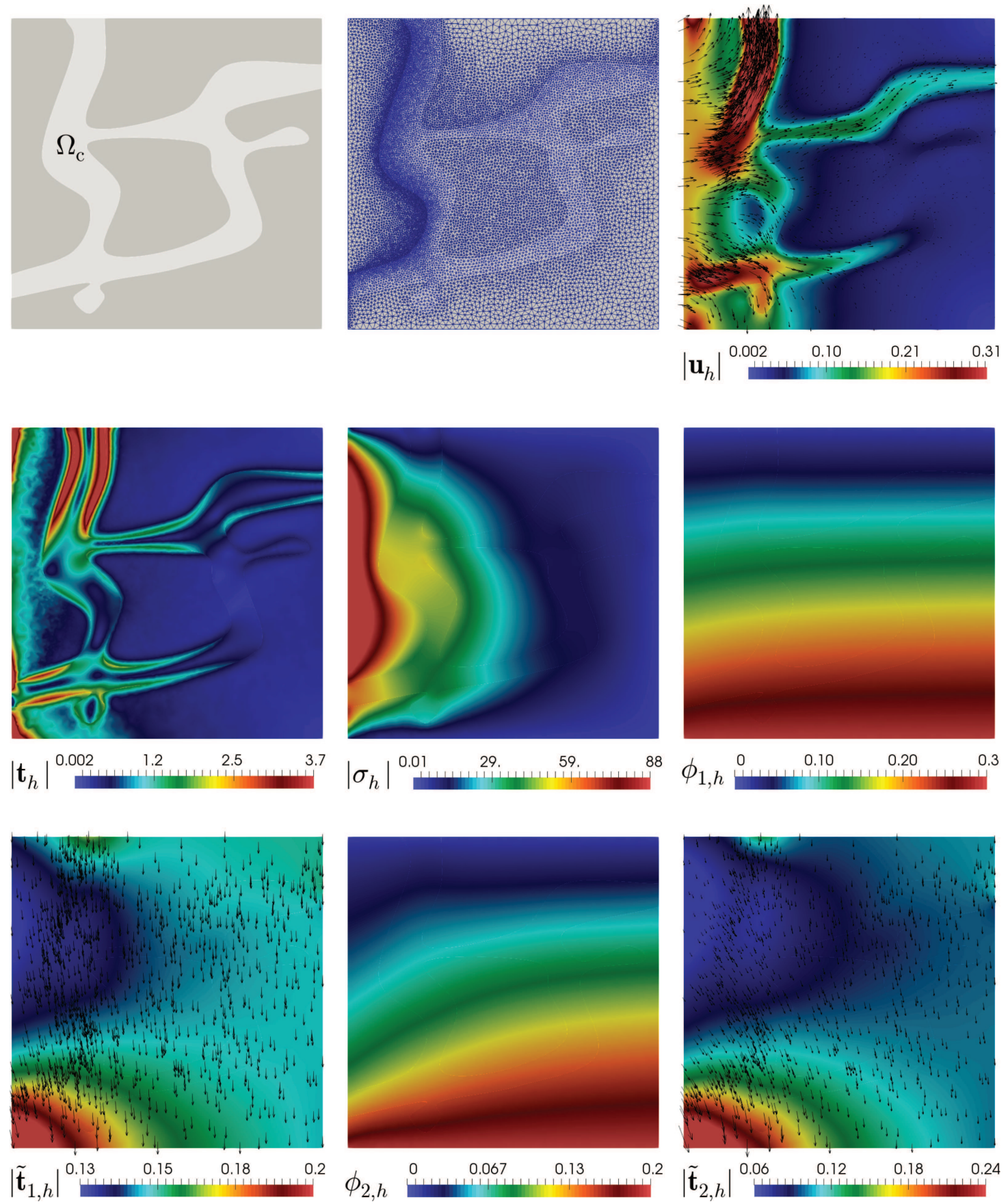

FiguRE 3. Example 3: Domain configuration, prescribed mesh, and computed magnitude of the velocity (top plots); computed magnitude of the velocity gradient and pseudostress tensor, and temperature field (middle plots); magnitude of the temperature gradient, concentration field, and magnitude of the concentration gradient (bottom plots). 
we have proposed for the coupling of the Brinkman-Forchheimer and double-diffusion equations has the ability to handle heterogeneous media using spatially varying parameters. Moreover, while this example is certainly the most challenging one, due to the strong jump discontinuity of the parameters across the two regions, we highlight that the numerical method (4.2) was able to handle it very efficiently. We notice that the mesh used in this example was built by considering an appropriate refinement around the interface that couples the porous medium with the channel network. Nevertheless, this refinement can be automatized by employing a suitable $a$ posteriori error indicator that captures the aforementioned discontinuity of the parameters. The corresponding a posteriori error analysis and numerical implementation will be addressed in a future work.

We end this paper with a comparison between the present approach and the one from [14] in terms of the corresponding DOF involved and the number of unknowns that they approximate. Indeed, while at first glance the fully-mixed method (4.2) seems more expensive than its augmented mixed counterpart from [14], we stress that the increase observed in the number of unknowns of the Galerkin scheme (4.2) with respect to that from [14] for the same mesh, is due to the fact that, differently from the latter, the former provides direct approximations to three additional variables of physical interest as well, namely the velocity gradient tensor $\mathbf{t}$, the temperature gradient vector $\widetilde{\mathbf{t}}_{1}$, and the concentration gradient vector $\widetilde{\mathbf{t}}_{2}$, in addition to yielding the possibility of employing a post-processing formula to recover the pressure (6.1). Moreover, these four further approximations hold with the same rate of convergence of the remaining variables. However, if one wanted to use the method from [14] to approximate the aforementioned extra unknowns, then one would need to employ numerical differentiation, which, as we know, leads to loss of accuracy of the respective computations.

Acknowledgements. This work has been partially supported by ANID-Chile through projects ACE 210010, CENTRO DE Modelamiento Matemático (FB 210005), Anillo of Computational Mathematics for Desalination Processes (ACT 210087), PAI77190084 of the PAI Program: Convocatoria Nacional Subvención a la Instalación en la Academia, and BECAS/DOCTORADO NACIONAL 21201539; and by Centro de Investigación en Ingeniería Matemática (CI $\left.{ }^{2} \mathrm{MA}\right)$, Universidad de Concepción.

\section{REFERENCES}

[1] A.K. Alzahrani, Importance of Darcy-Forchheimer porous medium in 3D convective flow of carbon nanotubes. Phys. Lett. A 382 (2018) 2938-2943.

[2] I. Ambartsumyan, E. Khattatov, T. Nguyen and I. Yotov, Flow and transport in fractured poroelastic media. GEM Int. J. Geomath. 10 (2019) 34.

[3] J.W. Barrett and W.B. Liu, Finite element approximation of the p-Laplacian. Math. Comput. 61 (1993) 523-537.

[4] G.A. Benavides, S. Caucao, G.N. Gatica and A.A. Hopper, A Banach spaces-based analysis of a new mixed-primal finite element method for a coupled flow-transport problem. Comput. Methods Appl. Mech. Eng. 371 (2020) 113285.

[5] G.A. Benavides, S. Caucao, G.N. Gatica and A.A. Hopper, A new non-augmented and momentum-conserving fully-mixed finite element method for a coupled flow-transport problem. Preprint 2020-21, Centro de Investigación en Ingeniería Matemática $\left(\mathrm{CI}^{2} \mathrm{MA}\right)$, Universidad de Concepción, Chile (2020).

[6] M.M. Bhatti, A. Zeeshan, R. Ellahi and G.C. Shit, Mathematical modeling of heat and mass transfer effects on MHD peristaltic propulsion of two-phase flow through a Darcy-Brinkman-Forchheimer porous medium. Adv. Powder Tech. 29 (2018) 11891197.

[7] F. Brezzi and M. Fortin, Mixed and Hybrid Finite Element Methods. Vol. 15 of Springer Series in Computational Mathematics. Springer-Verlag, New York (1991).

[8] H.C. Brinkman, A calculation of the viscous force exerted by a flowing fluid on a dense swarm of particles. Appl. Sci. Res. 1 (1949) 27-34.

[9] R. Bürger, P.E. Méndez and R. Ruiz-Baier, On $\mathbf{H}($ div)-conforming methods for double-diffusion equations in porous media. SIAM J. Numer. Anal. 57 (2019) 1318-1343.

[10] J. Camaño, C. Muñoz and R. Oyarzúa, Numerical analysis of a dual-mixed problem in non-standard Banach spaces. Electron. Trans. Numer. Anal. 48 (2018) 114-130.

[11] J. Camaño, C. García and R. Oyarzúa, Analysis of a conservative mixed-FEM for the stationary Navier-Stokes problem. Numer. Methods Part. Differ. Equ. 37 (2021) 2895-2923.

[12] S. Caucao and I. Yotov, A Banach space mixed formulation for the unsteady Brinkman-Forchheimer equations. IMA J. Numer. Anal. 41 (2021) 2708-2743.

[13] S. Caucao, M. Discacciati, G.N. Gatica and R. Oyarzúa, A conforming mixed finite element method for the NavierStokes/Darcy-Forchheimer coupled problem. ESAIM: M2AN 54 (2020) 1689-1723. 
[14] S. Caucao, G.N. Gatica, R. Oyarzúa and N. Sánchez, A fully-mixed formulation for the steady double-diffusive convection system based upon Brinkman-Forchheimer equations. J. Sci. Comput. 85 (2020) 37.

[15] S. Caucao, R. Oyarzúa and S. Villa-Fuentes, A new mixed-FEM for steady-state natural convection models allowing conservation of momentum and thermal energy. Calcolo 57 (2020) 39.

[16] A.O. Celebi, V.K. Kalantarov and D. Ugurlu, On continuous dependence on coefficients of the Brinkman-Forchheimer equations. Appl. Math. Lett. 19 (2006) 801-807.

[17] E. Colmenares, G.N. Gatica and R. Oyarzúa, Fixed point strategies for mixed variational formulations of the stationary Boussinesq problem. C. R. Math. Acad. Sci. Paris 354 (2016) 57-62.

[18] E. Colmenares, G.N. Gatica and S. Moraga, A Banach spaces-based analysis of a new fully-mixed finite element method for the Boussinesq problem. ESAIM: M2AN 54 (2020) 1525-1568.

[19] E. Colmenares, G.N. Gatica, S. Moraga and R. Ruiz-Baier, A fully-mixed finite element method for the steady state OberbeckBoussinesq system. SMAI J. Comput. Math. 6 (2020) 125-157.

[20] T.A. Davis, Algorithm 832: UMFPACK V4.3 - an unsymmetric-pattern multifrontal method. ACM Trans. Math. Softw. 30 (2004) 196-199.

[21] A. Ern and J.-L. Guermond, Theory and Practice of Finite Elements. Vol. 159 of Applied Mathematical Sciences. SpringerVerlag, New York, 2004.

[22] J. Faulkner, B.X. Hu, S. Kish and F. Hua, Laboratory analog and numerical study of ground water flow and solute transport in a karst aquifer with conduit and matrix domains. J. Contam. Hydrol. 110 (2009) 34-44.

[23] P. Forchheimer, Wasserbewegung durch boden. Z. Ver. Deutsch Ing. 45 (1901) 1782-1788.

[24] G.N. Gatica, A Simple Introduction to the Mixed Finite Element Method: Theory and Applications. SpringerBriefs in Mathematics. Springer, Cham (2014).

[25] G.N. Gatica, G.C. Hsiao and S. Meddahi, Further developments on boundary-field equation methods for nonlinear transmission problems. J. Math. Anal. Appl. 502 (2021) 125262.

[26] V. Girault and P.A. Raviart, Finite Element Methods for Navier-Stokes Equations: Theory and Algorithms. Vol. 5 of Springer Series in Computational Mathematics. Springer-Verlag, Berlin (1986).

[27] F. Hecht, New development in FreeFem++. J. Numer. Math. 20 (2012) 251-265.

[28] P.N. Kaloni and J. Guo, Steady nonlinear double-diffusive convection in a porous medium based upon the BrinkmanForchheimer model. J. Math. Anal. Appl. 204 (1996) 138-155.

[29] M. Ôtani and S. Uchida, Global solvability of some double-diffusive convection system coupled with Brinkman-Forchheimer equations. Lib. Math. (N.S.) 33 (2013) 79-107.

[30] A. Quarteroni and A. Valli, Numerical Approximation of Partial Differential Equations. Vol. 23 of Springer Series in Computational Mathematics. Springer-Verlag, Berlin (1994).

[31] S. Safi and S. Benissaad, Double-diffusive convection in an anisotropic porous layer using the Darcy-Brinkman-Forchheimer formulation. Arch. Mech. (Arch. Mech. Stos.) 70 (2018) 89-102.

[32] Y.J. Zhuang, H.Z. Yu and Q.Y. Zhu, A thermal non-equilibrium model for 3D double diffusive convection of power-law fluids with chemical reaction in the porous medium. Int. J. Heat Mass Trans. 115-B (2017) 670-694.

\section{Subscribe to Open (S2O) A fair and sustainable open access model}

This journal is currently published in open access under a Subscribe-to-Open model (S2O). S2O is a transformative model that aims to move subscription journals to open access. Open access is the free, immediate, online availability of research articles combined with the rights to use these articles fully in the digital environment. We are thankful to our subscribers and sponsors for making it possible to publish this journal in open access, free of charge for authors.

\section{Please help to maintain this journal in open access!}

Check that your library subscribes to the journal, or make a personal donation to the S2O programme, by contacting subscribers@edpsciences.org

More information, including a list of sponsors and a financial transparency report, available at: https://www. edpsciences.org/en/maths-s2o-programme 